SAND 93-0187

Unlimited Release

SAND--93-0187

Printed March 1993

\title{
Plasma-Etching Science Meets Technology in the MDL
}

K. E. Greenberg and P. A. Miller

Laser, Optics, and Remote Sensing Department

R. Patteson and B. K. Smith

Silicon IC Patterning Department

Sandia National Laboratories

Albuquerque, New Mexico 87185

\section{Executive Summary}

In recent work at Sandia National Laboratories, the results from fundamental investigations of low-temperature plasma systems were used to improve chamber-to-chamber reproducibility and reliability in commercial plasma-etching equipment. This work is an excellent example of how Sandia can make an impact in areas of importance both to national defense and to domestic industry (dual use technologies). It spotlights a unique capability of the Labs in the area of microelectronics: the ability to conduct fundamental research with experimental laboratory equipment and to apply the results of that research to commercial, "in-house" equipment. 


\section{Acknowledgments}

This work would not have been possible without the support of many people. We thank Ben Aragon, Dawn Chavez, Mike Cook, Beth Gonzales, Tom Gugliotta, Paul Pochan, and George Roth for their excellent technical assistan se. SEMATECH and, in particular, Dick Clover, Ron Horwath and Terry Turner, played an important role in the initiation and funding of this work. We greatly appreciate the encouragement and backing provided by Jim Gerardo and Harry Weaver; they made the whole thing possible. We applaud the Herculean effort of Phil Hargis in the design of the GEC Reference Cell. Thanks also go to Joe Martinez and the Office of Basic Energy Sciences for supporting our fundamental discharge studies under contract KC-03-01-03. This work was performed at Sandia National Laboratories, supported by the U.S. Department of Energy under contract DE-AC04-76DP00789 and by SEMATECH under the SETEC (Semiconductor Equipment Technology Center) program. 


\begin{abstract}
Results from fundamental investigations of low-temperature plasma systems were used to improve chamber-to-chamber reproducibility and reliability in commercial plasma-etching equipment. The fundamental studies were performed with a GEC RF Reference Cell, a laboratory research system designed to facilitate experimental and theoretical studies of plasma systems. Results and diagnostics from the Reference Cell studies were then applied to analysis and rectification of chamber-to-chamber variability on a commercial, multichamber, plasma reactor. Pertinent results were transferred to industry.
\end{abstract}




\section{Table of Contents}

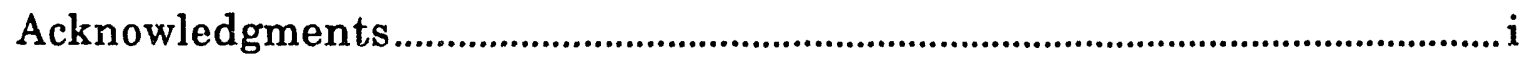

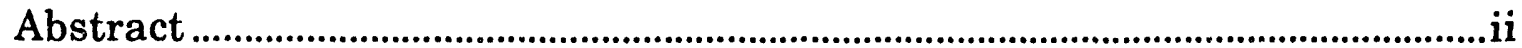

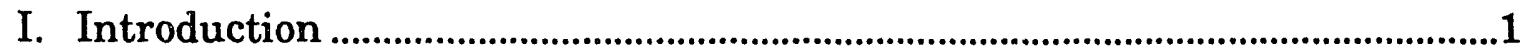

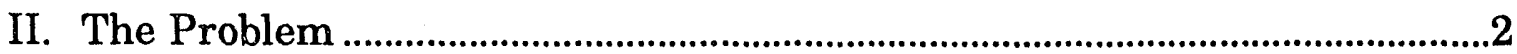

III. Application of GEC Cell Results to Commercial Equipment...................3

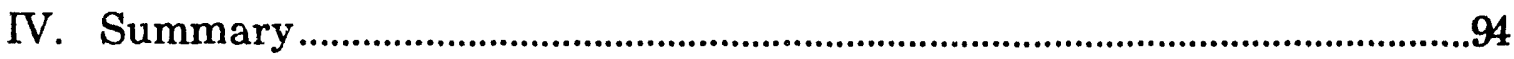




\section{Introduction}

One of the most important commercial applications of low-temperature glow discharges is the fabrication of microelectronic circuits. Lowtemperature plasmas are indispensable for etching the fine features needed in large-scale device integration. These plasmas are also used to deposit materials, remove photoresist, and enhance oxidation.

Unfortunately, many of the plasma-based processes used to produce integrated circuits suffer reliability problems. A process may work well for some time and then fail mysteriously. A process "recipe" (gas flow, pressure, power, etc.) may yield certain results in one machine and completely different results in another. These types of problems cause decreased efficiency and increased costs for the semiconductor industry.

Optimization and control of the highly complex plasma systems used in the semiconductor industry have been limited by an incomplete understanding of the underlying chemistry and physics of the plasma. Due to its extensive resources, Sandia National Laboratories (SNL) is uniquely situated both to conduct fundamental investigations of the plasma and to apply the results of these studies to commercial processes. SNL has strong diagnostic capabilities (electrical, optical/laser, microwave, etc.) for analyzing plasma systems. In addition, the Microelectronics Development Lab (MDL) at SNL contains a large Class-1 clean-room facility with commercial plasma equipment for semiconductor device fabrication. Unlike the situatior in most semiconductor companies, research can be conducted with the $t_{i}$ iline equipment in the MDL as well as with experimental laborato - $_{j}$ equipment.

In recent projects at SNL, we performed fundamental investigations of plasma systems and used the results of these studies to analyze and rectify problems with commercial plasma-etching equipment. These projects included work supported by SEMATECH and by SNL Organizations 1100 and 1300. Part of this work was conducted as a joint 1100/1300 program. This report summarizes some of these research efforts. In particular, the report concentrates on work conducted to improve chamber-to-chamber (or machine-to-machine) reproducibility and reliability in plasma-etching equipment.

The remainder of this document is divided into three sections. Section II provides background material; it describes a problem encountered with commercial plasma-etching equipment and our approach to rectifying this problem. Section III, in which we present the results of the studies, is a review of a joint 1100/1300 seminar presented in October, 1992. This section contains copies of the overhead transparencies from the presentation along with additional explanatory comments. The final section contains a brief summary. 


\section{The Problem}

The parallel-plate radio-frequency (rf) diode, operating at $13.56 \mathrm{MHz}$, is the discharge configuration most commonly used for plasma etching. Figure 1 shows a schematic diagram of this configuration.

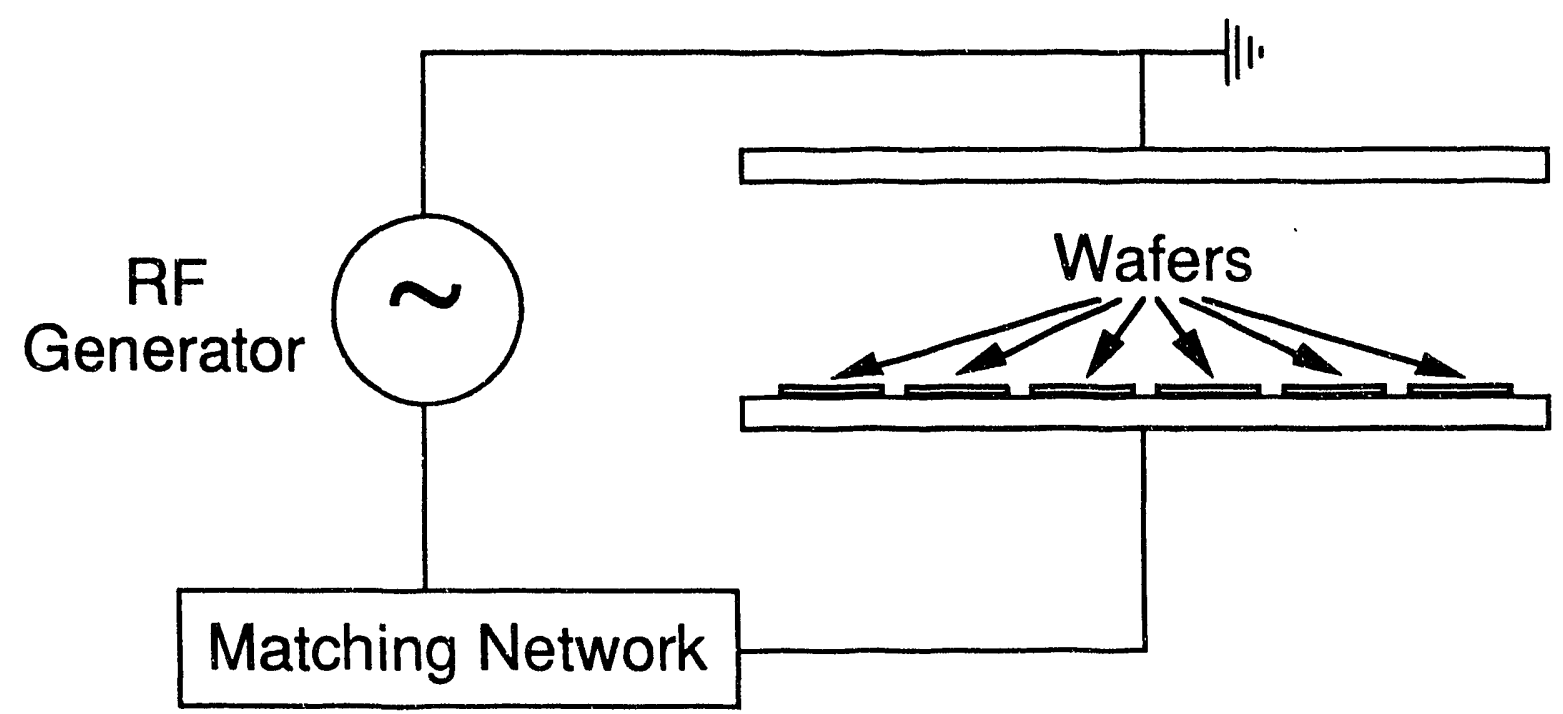

Fig. 1. Schematic diagram of a parallel-plate plasma-etching system.

The parallel-plate rf diode is characterized by two electrodes that typically are separated by 5 to $40 \mathrm{~mm}$. An impedance matching network couples power from an rf generator to one of the electrodes (in this case, the lower electrode in Fig. 1). The matching network contains a blocking capacitor that prevents the flow of direct current $(\mathrm{dc})$. The other electrode and the remainder of the etching chamber are maintained at ground potential. Due to the asymmetry of the grounded and powered surfaces of the etching chamber and the presence of the blocking capacitor in the matching network, a dc bias develops across the plasma. This bias accelerates positively charged ions toward the powered electrode. If wafers are placed on the powered electrode, highly directional etching can be obtained due to the directional nature of the ion bombardment.

When wafer diameters were small, a number of wafers were placed on the electrode and etched simultaneously. As wafer diameters increased, the semiconductor industry moved to single-wafer processing. A single-wafer processing reactor may have multiple etching chambers with each chamber etching one wafer at a time.

A serious problem, encountered by workers in the MDL and in the US semiconductor industry, is that supposedly identical etching chambers do 
not produce the same etch results for a given etch recipe. Not only has this phenomenon been observed for a given model line of etchers from a particular manufacturer, but it has also been observed for different, nominally identical chambers on a multichamber piece of equipment. As an example, two chambers on a Drytek Quad reactor in the MDL are devoted to silicon dioxide etching. For identical input conditions (gas flow, pressure, power, etc.), the etch rates in the two chambers differed by as much as $30 \%$. This performance variability makes process development more difficult and time consuming than necessary. Furthermore, there is another subtle, yet important, implication of process variability for process efficiency. If, in a production line, there is chamber-to-chamber variability, then typically one chamber will produce better overall results than the other chambers. To state it another way, with process variability most of the chambers are inferior in quality of product and/or throughput when compared to the "best" chamber. Therefore, process variability directly signifies inefficiency of the production line.

We investigated chamber-to-chamber variability and discovered solutions for the problem. Our initial studies were conducted with a research plasma chamber known as the "GEC RF Reference Cell." 1 The results from the research chamber were then applied to the Drytek multichamber system in the MDL. The next section of this report details the Reference Cell and Drytek work.

\section{Application of GEC Cell Results to Commercial Equipment}

As stated earlier, this section, which describes details of the GEC Reference Cell and our results with the Drytek reactor in the MDL, is a summary of a joint 1100/1300 seminar. It includes copies of the overhead transparencies from the talk along with additional comments about the transparencies. The transparencies are presented on the left (even-numbered) pages and the accompanying comments are presented on the opposite (odd-numbered) pages.

1"The GEC RF Reference Cell: Diagnostic Techniques and Initial Results," K. E. Greenberg, P. J. Hargis, Jr., and P. A. Miller, Sandia Report SETEC 90-013, November, 1990; "The GEC RF Reference Cell: A ParallelPlate Radio-Frequency System to Study Plasma-Processing Discharges," P. J. Hargis, Jr., et al., Accepted for publication in Rev. Sci. Instruments (1993). 

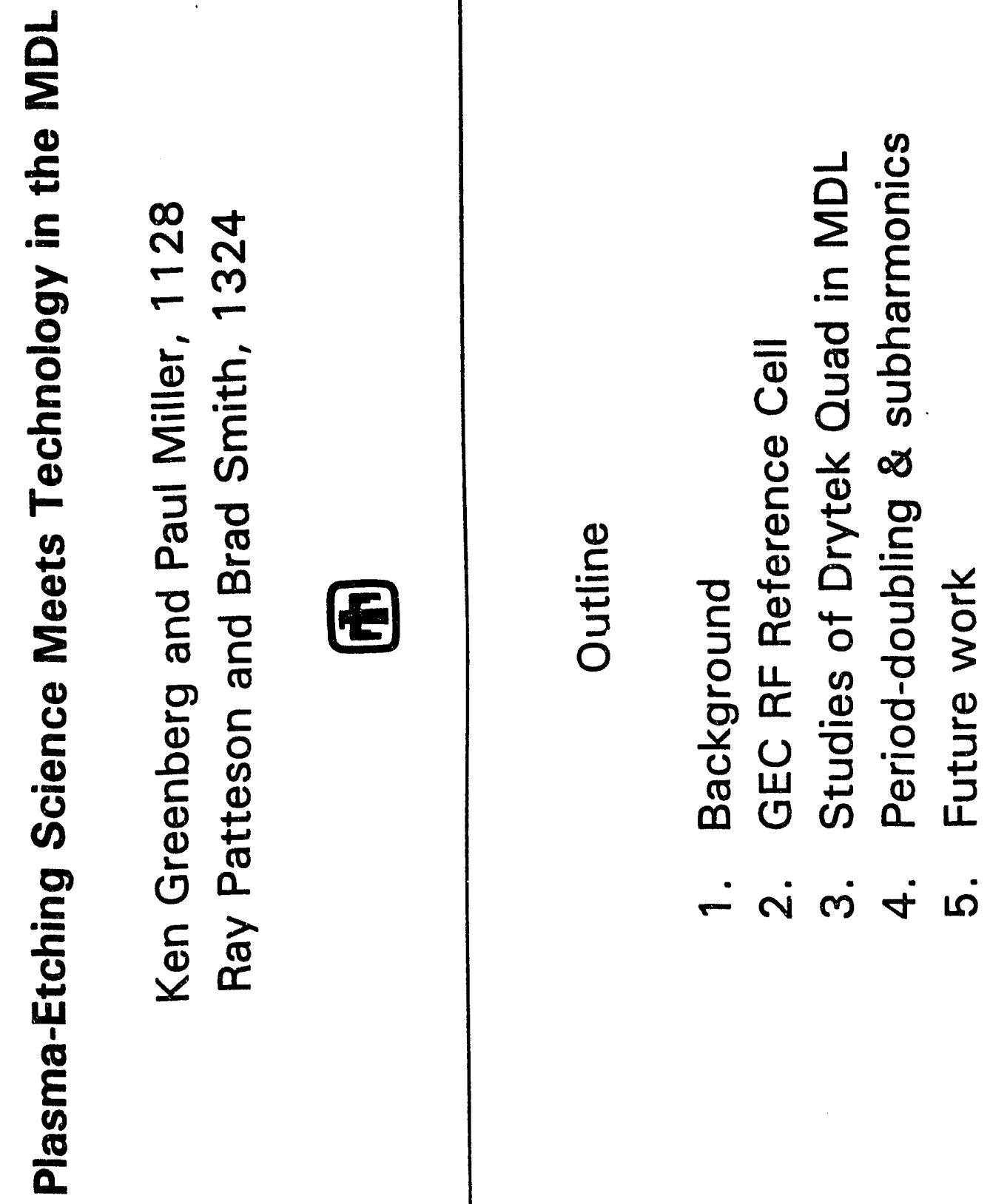


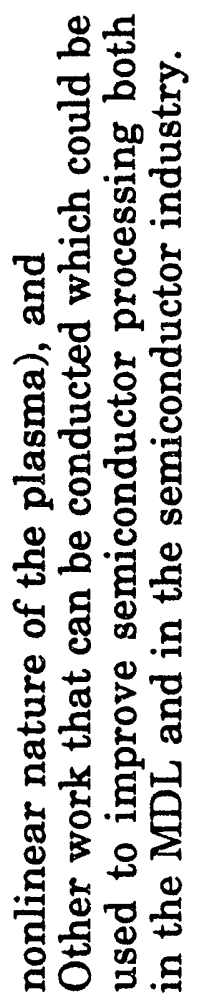

ลี
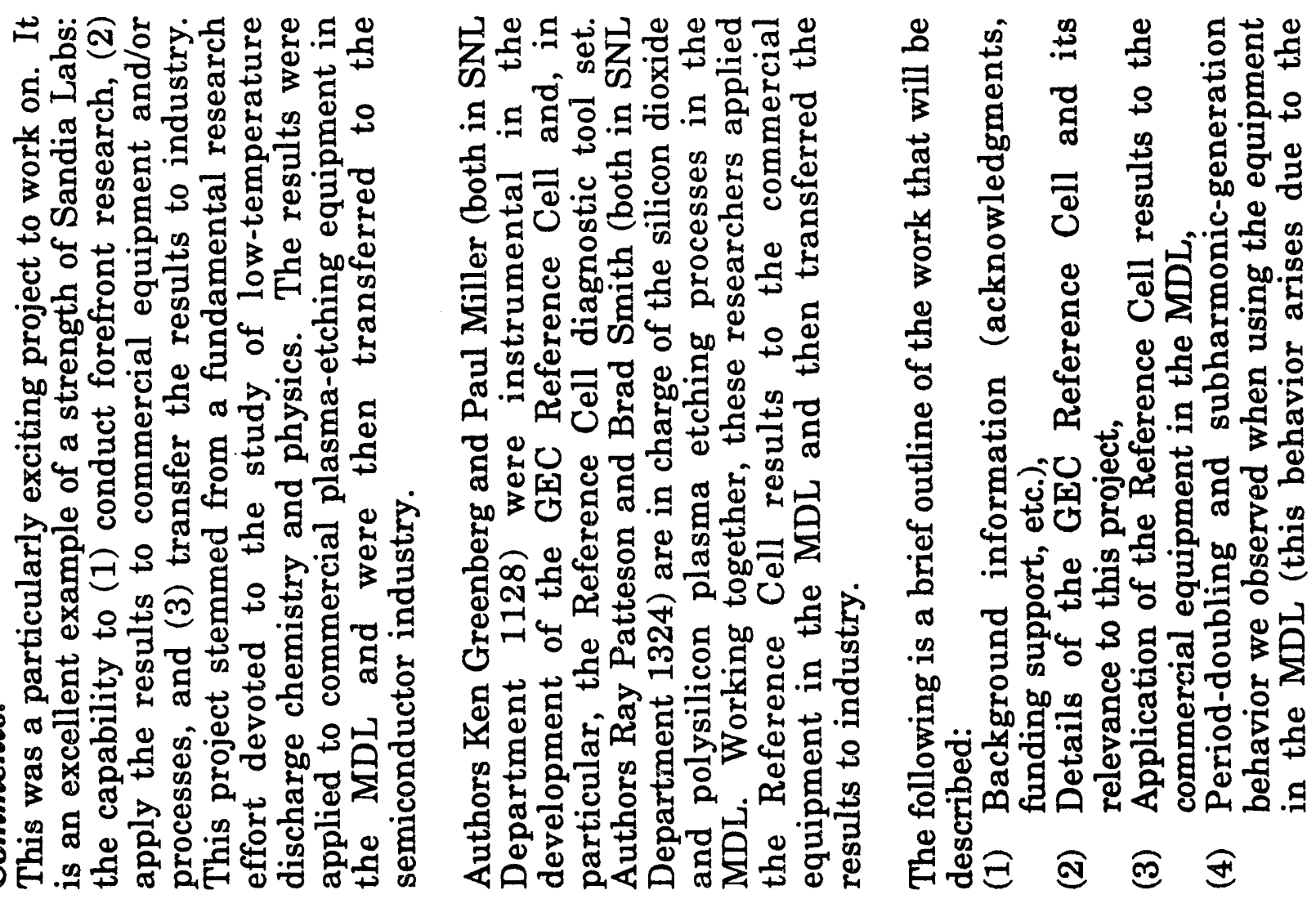


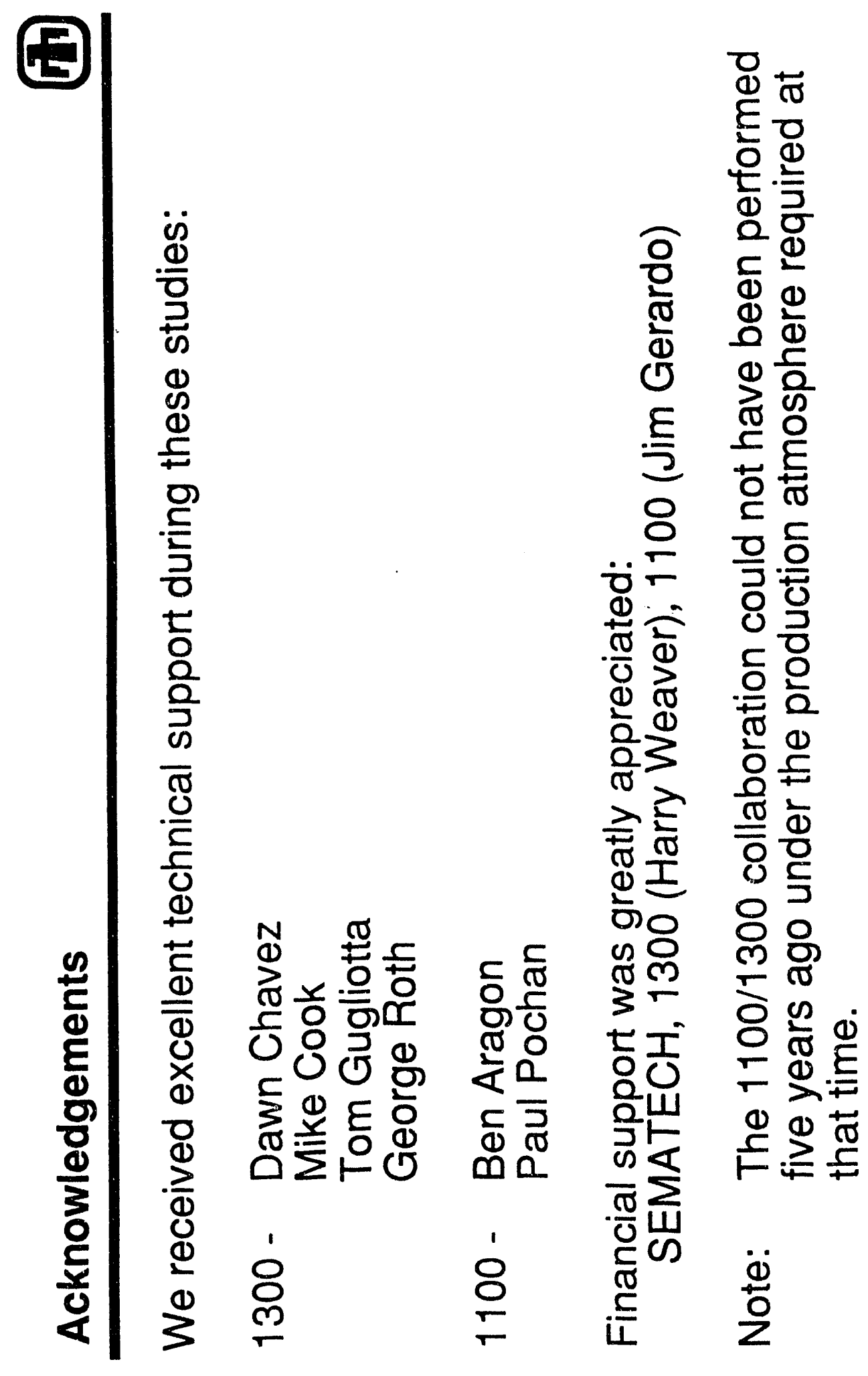




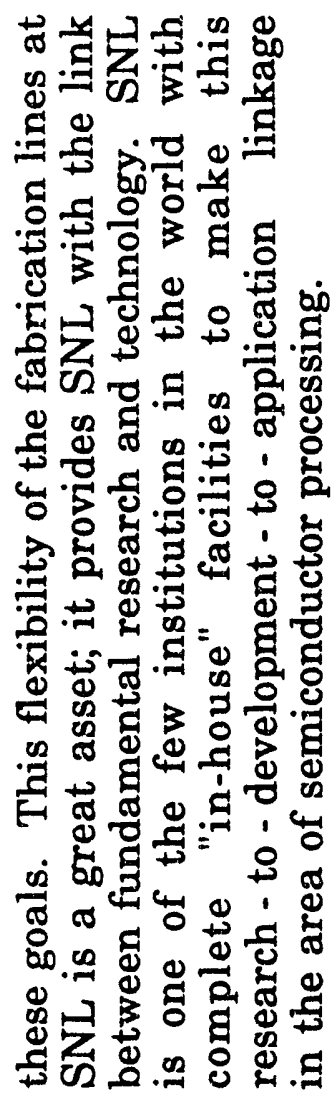

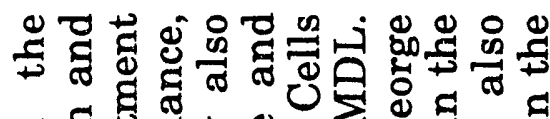

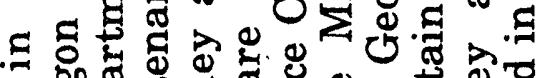
— ส

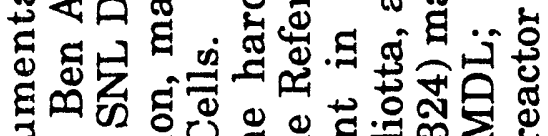

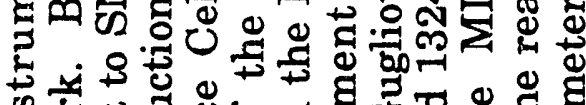
कै $\approx$ उ

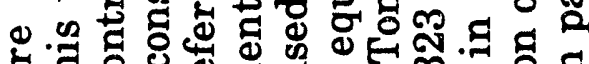
屯 공 ठ

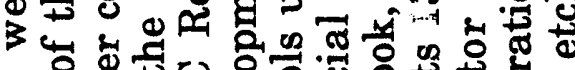

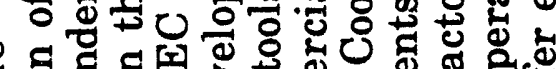

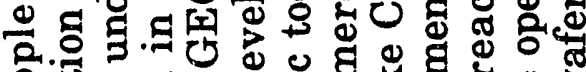

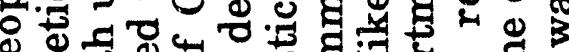

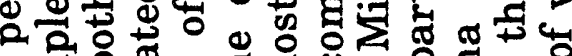

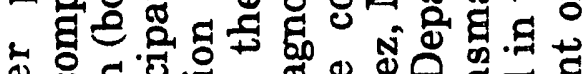
웡

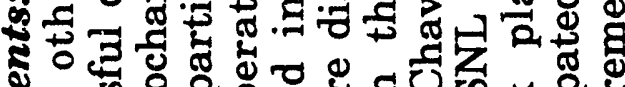

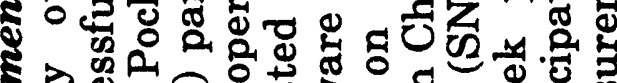

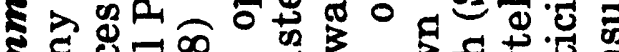

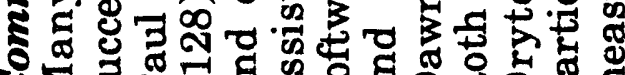

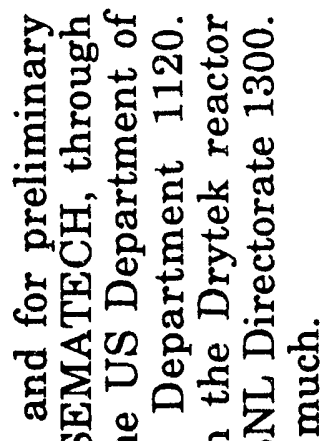
此

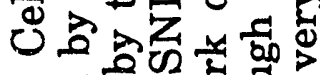

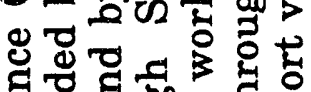

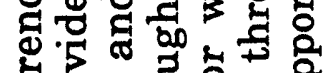

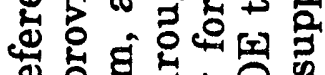
के है

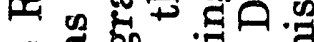
\&

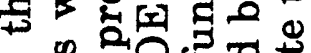
- क

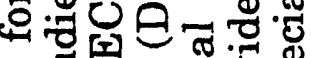
此证国

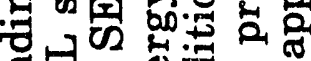

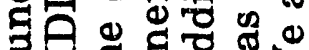

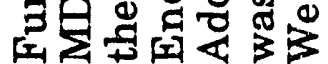

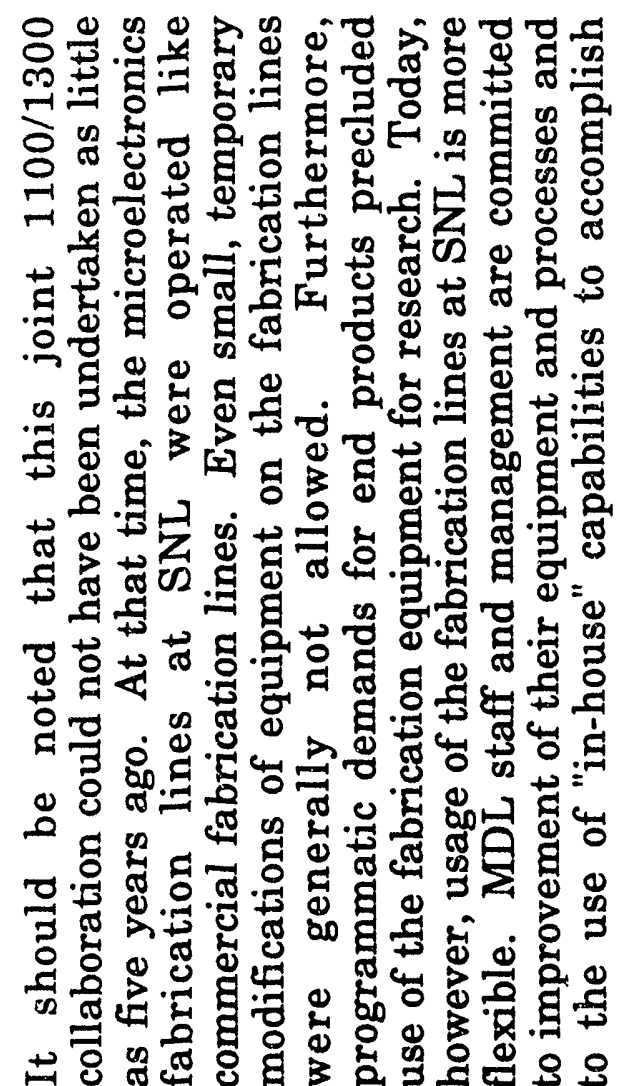




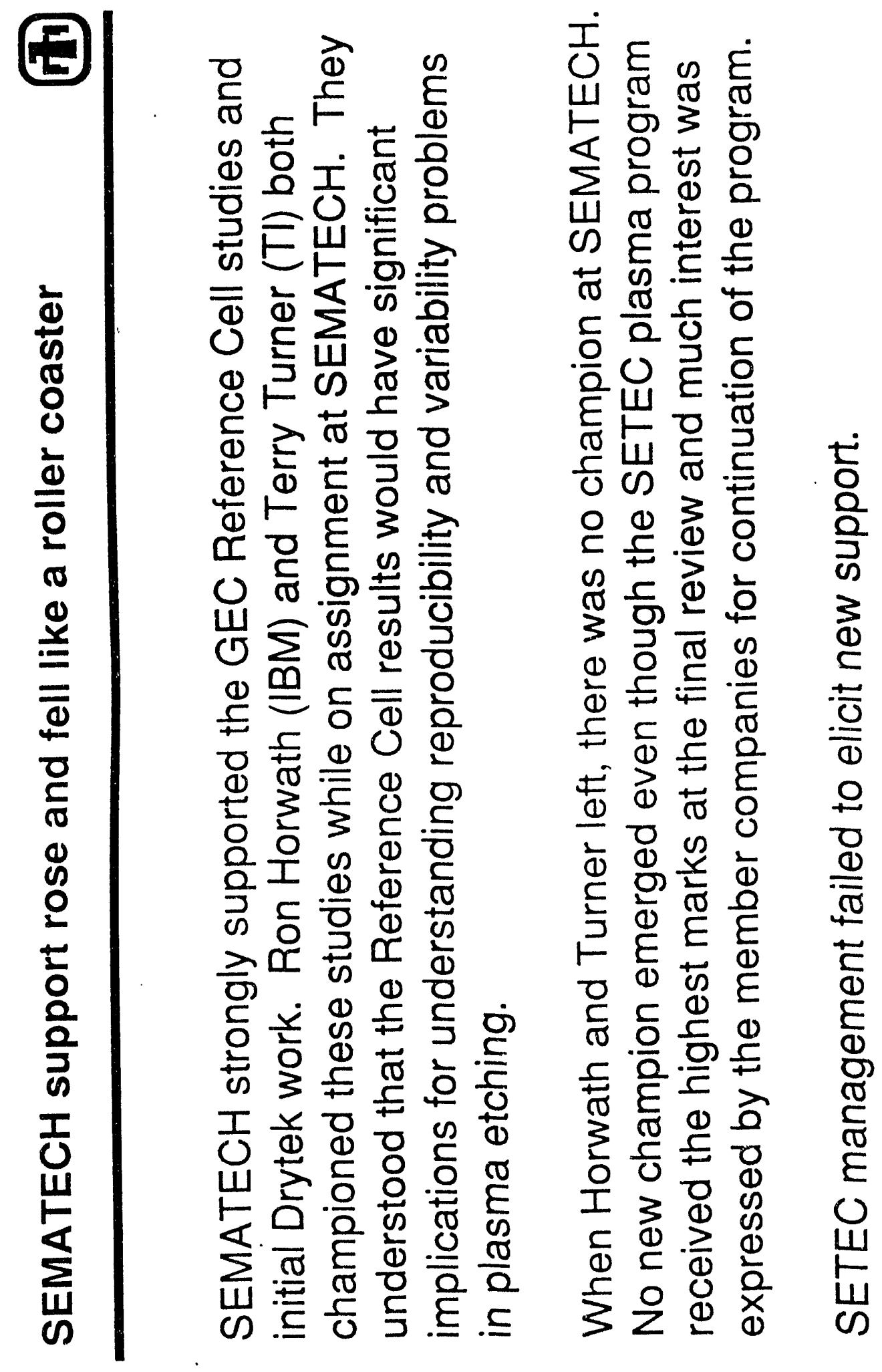




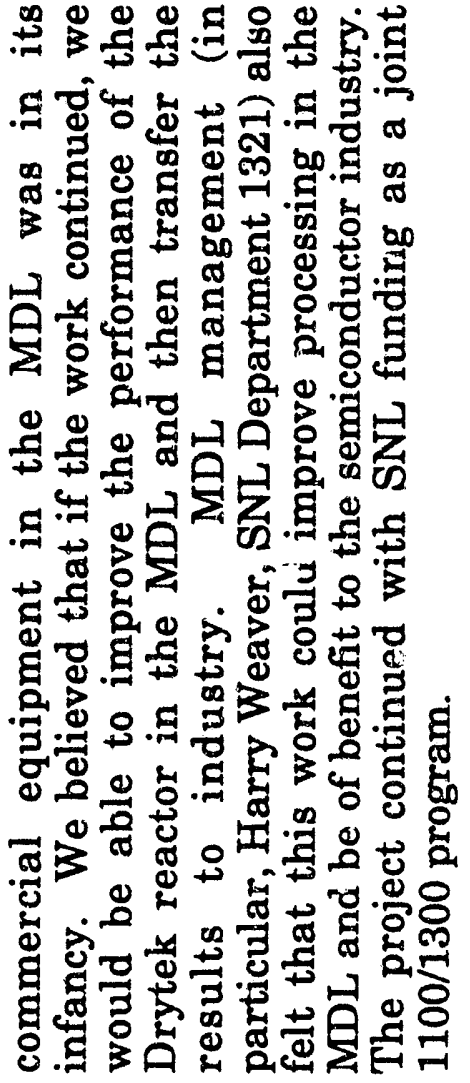

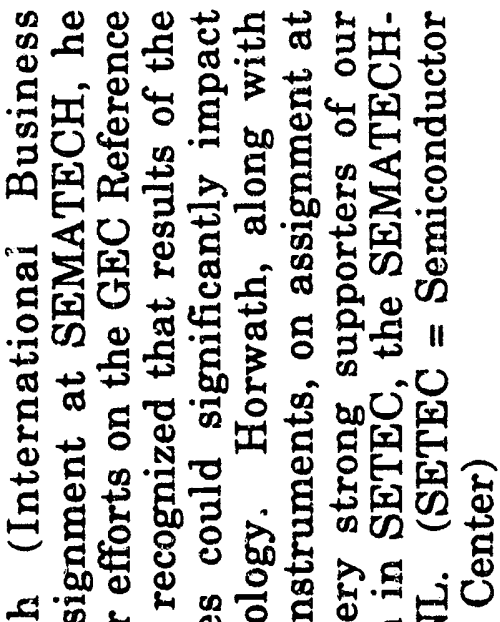

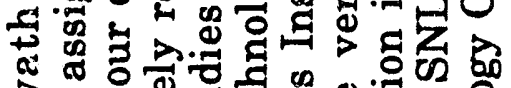

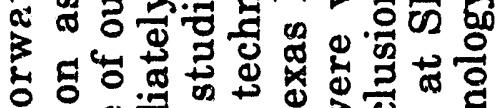

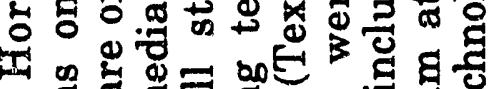
क สี \%ี

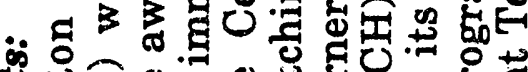

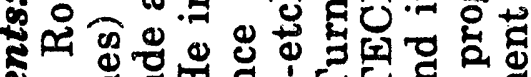
ฐ.

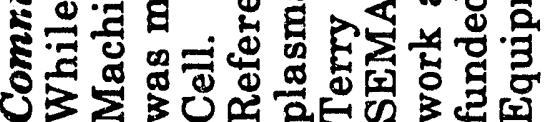

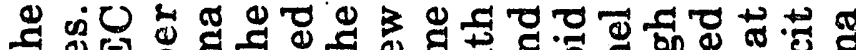

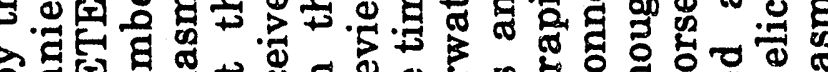
ล ర명

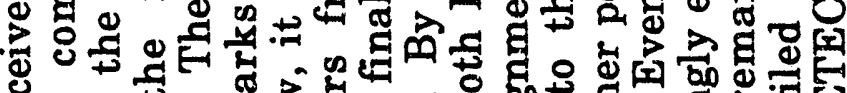

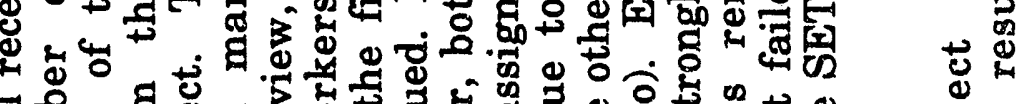

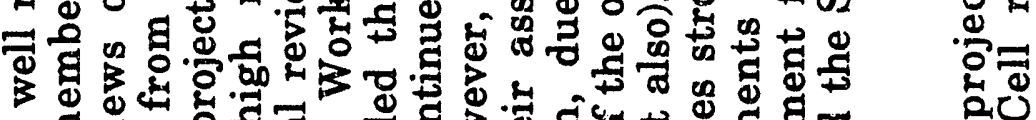
ด ㅂ. б

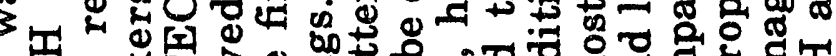
出二

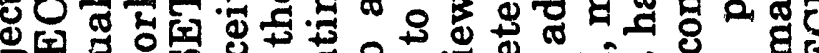

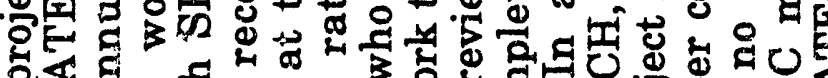
运

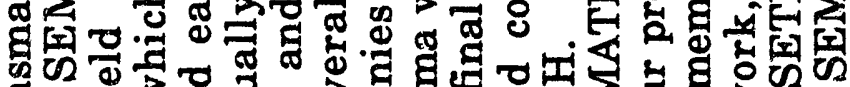

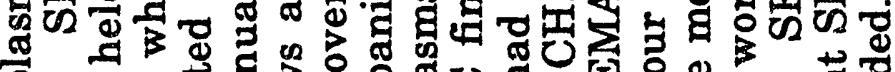

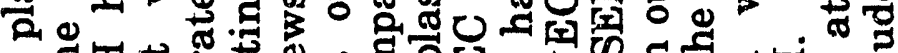

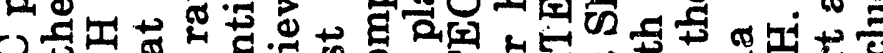

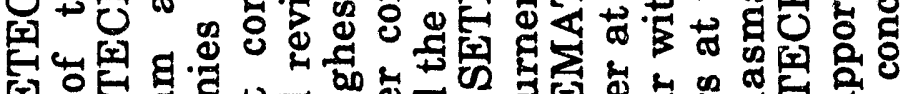

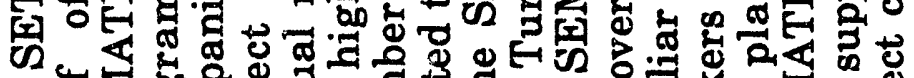

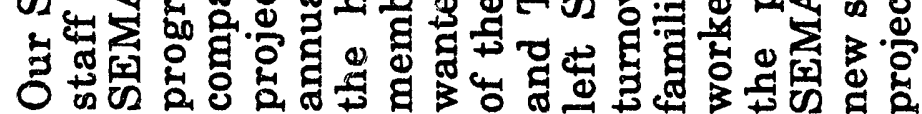

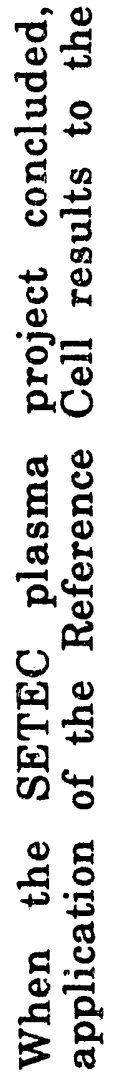




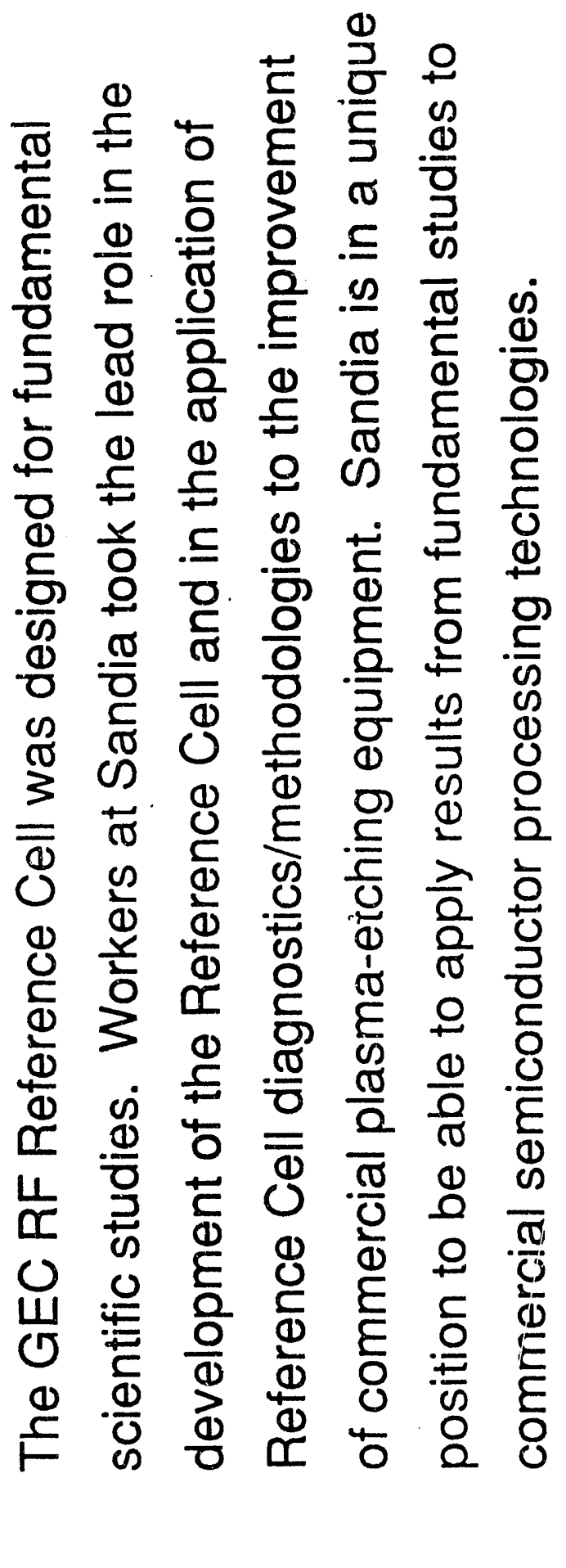




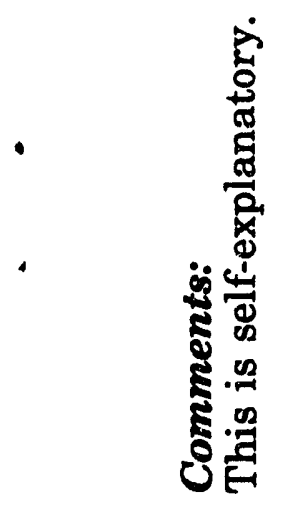



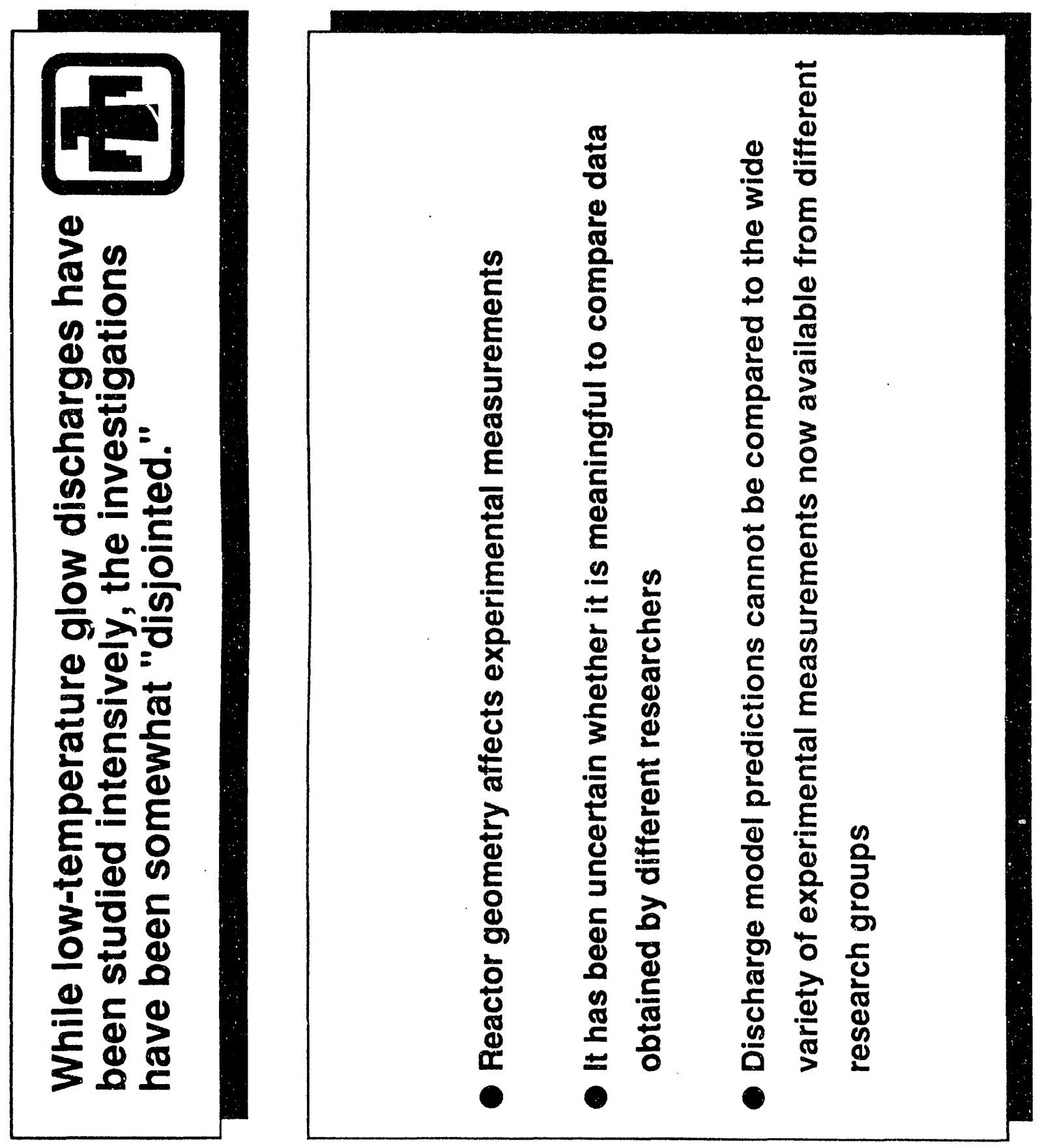


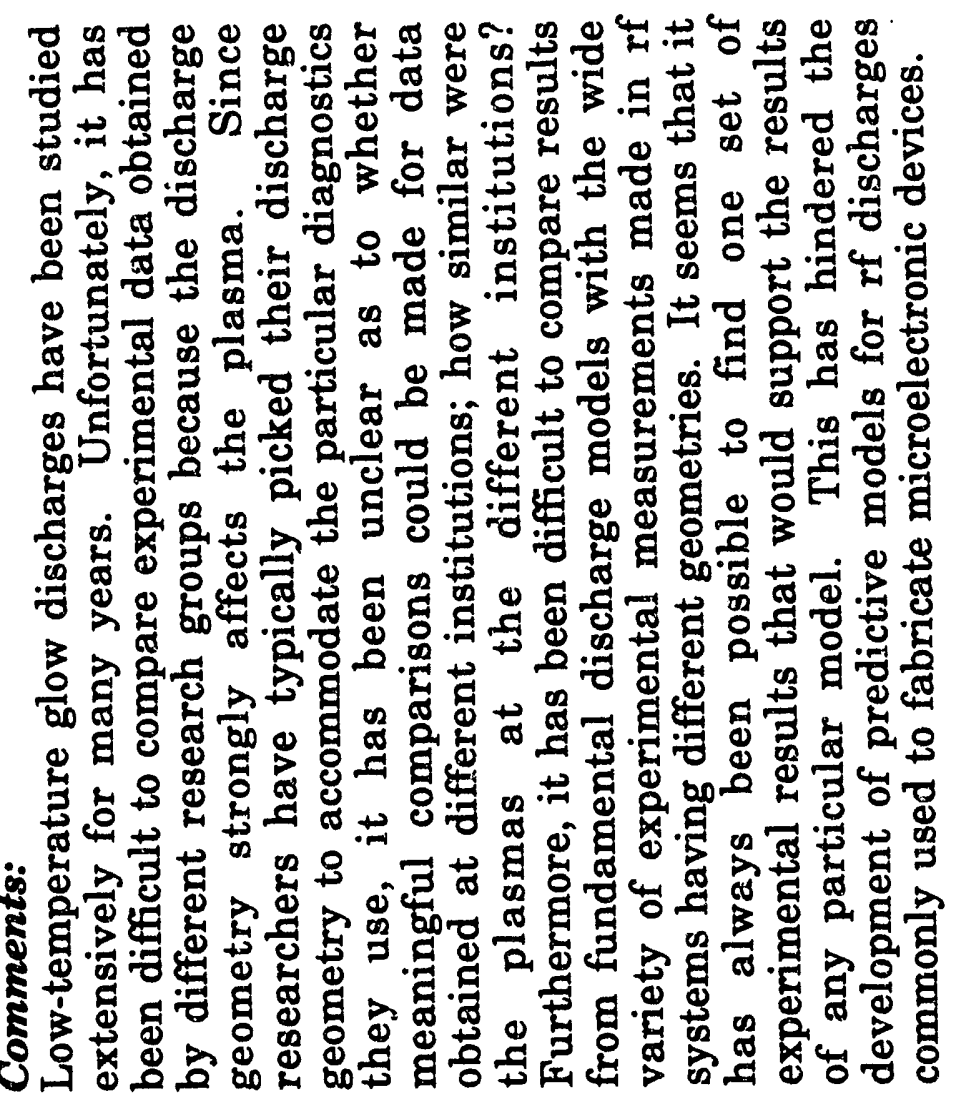




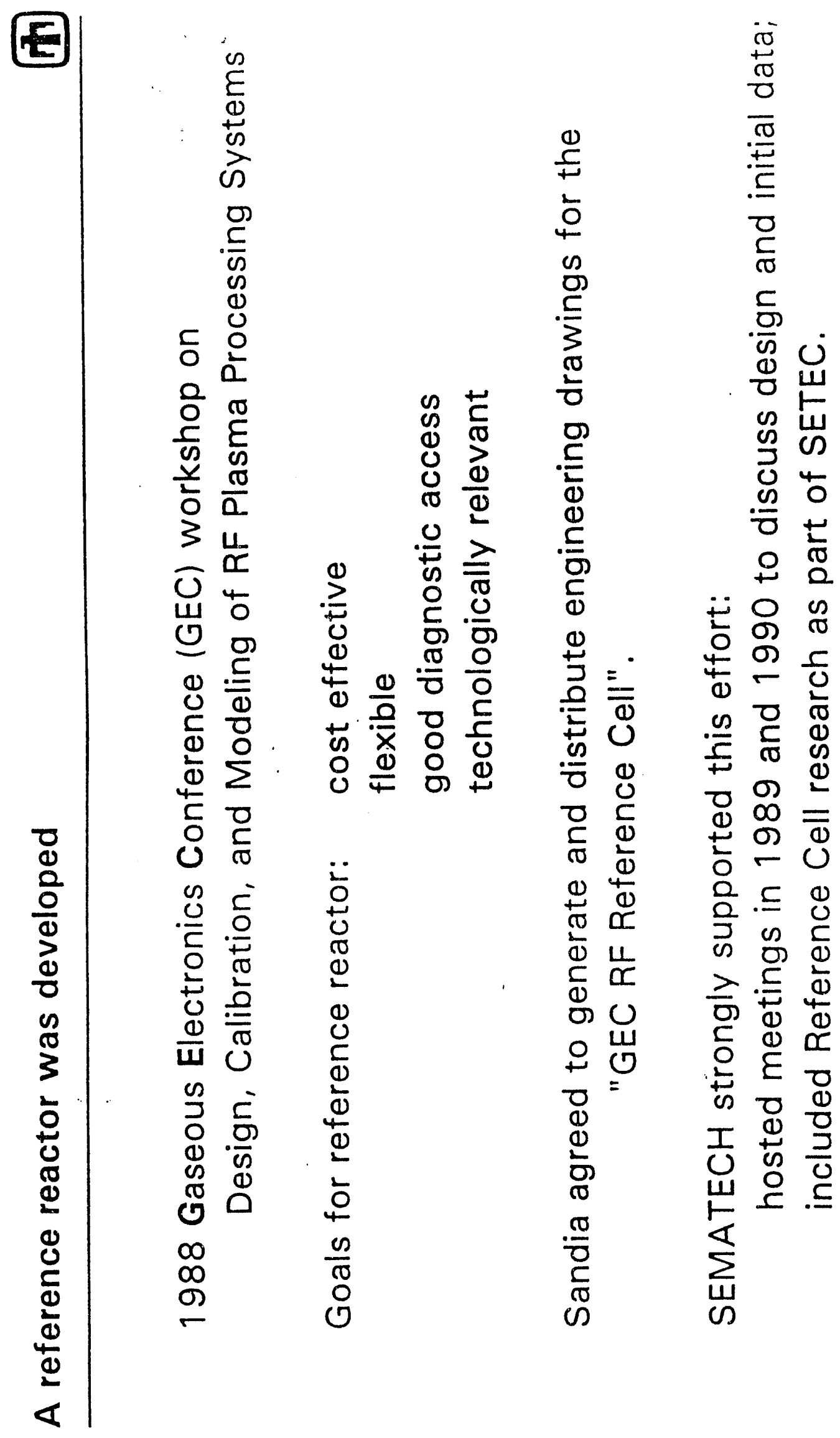




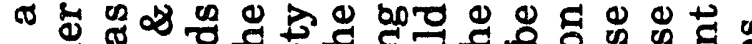

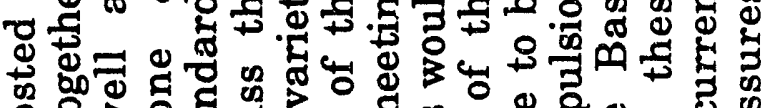

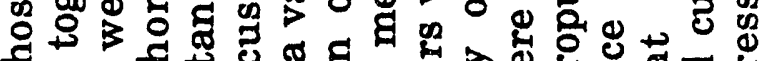

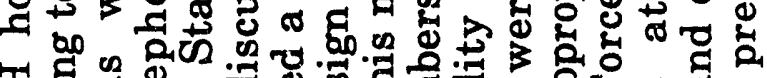
舟 包过

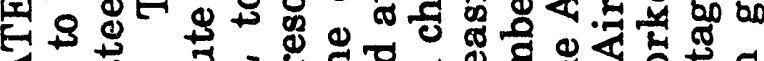

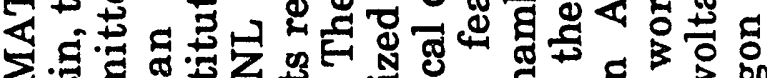

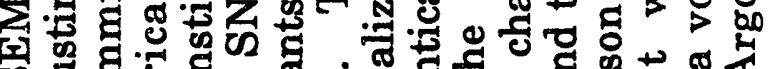

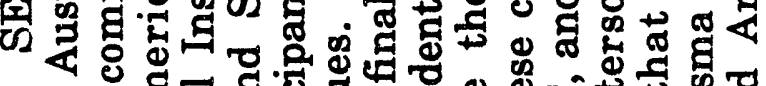

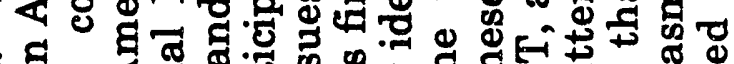

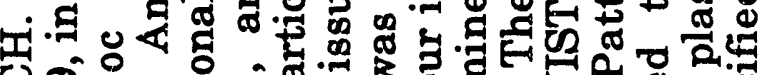
ช ज्ञ

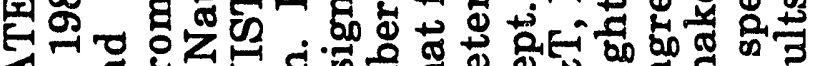

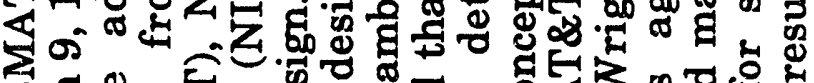

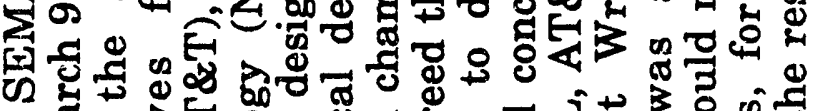

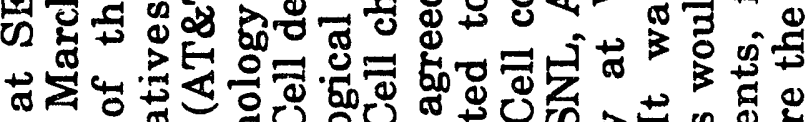
४⿻一𠃋十

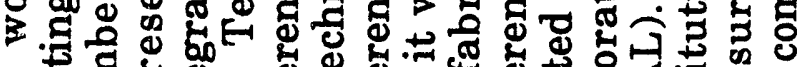

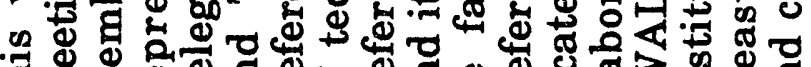

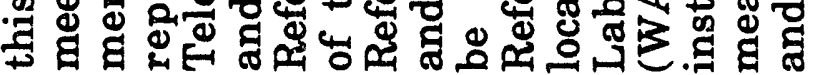

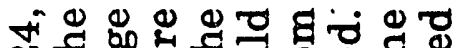
ง

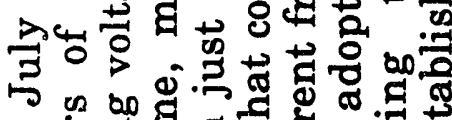
ต क ส 蛋

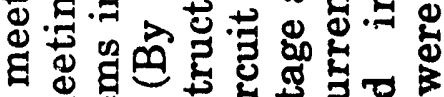

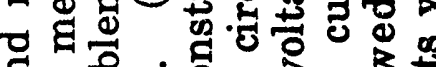

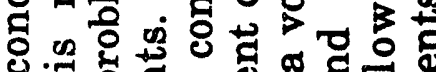

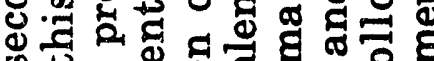
叶政 ๘

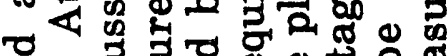
¿

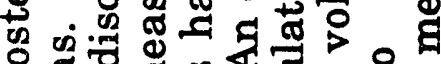

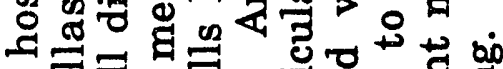

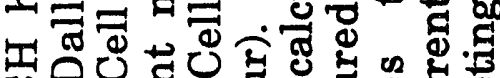
乙ค团 되

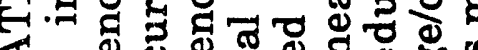
క

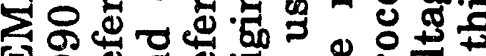

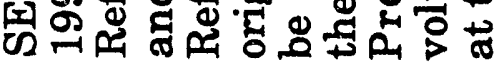

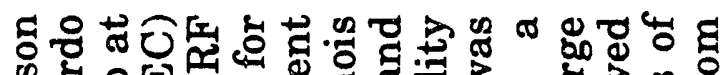

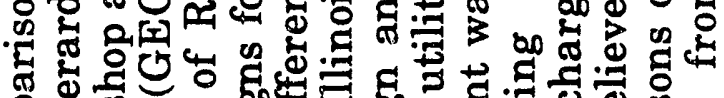

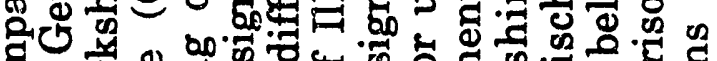

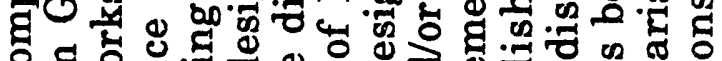

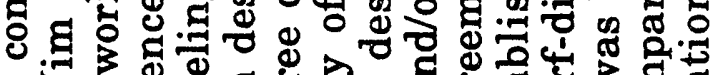
๙ง

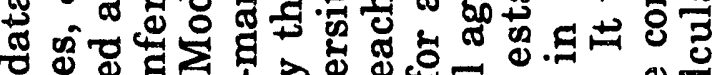
○

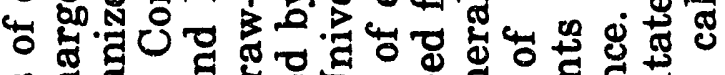

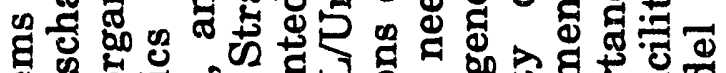

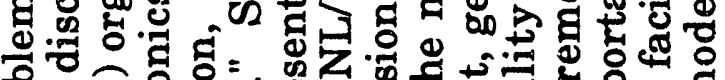
응 웅웡

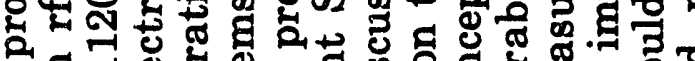

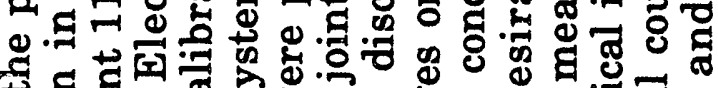
ஸ

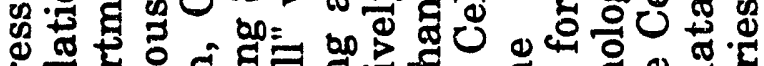

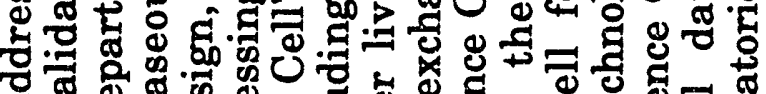

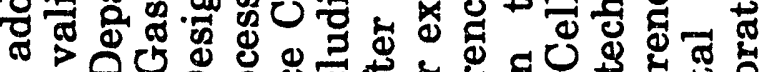

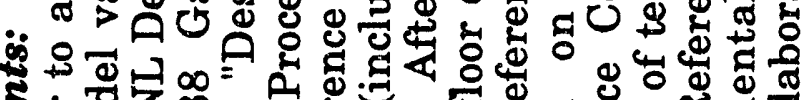

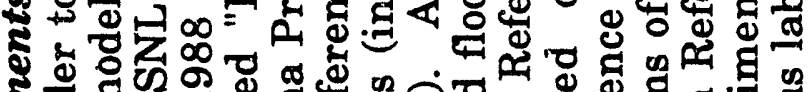

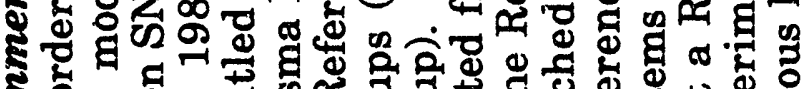
ह

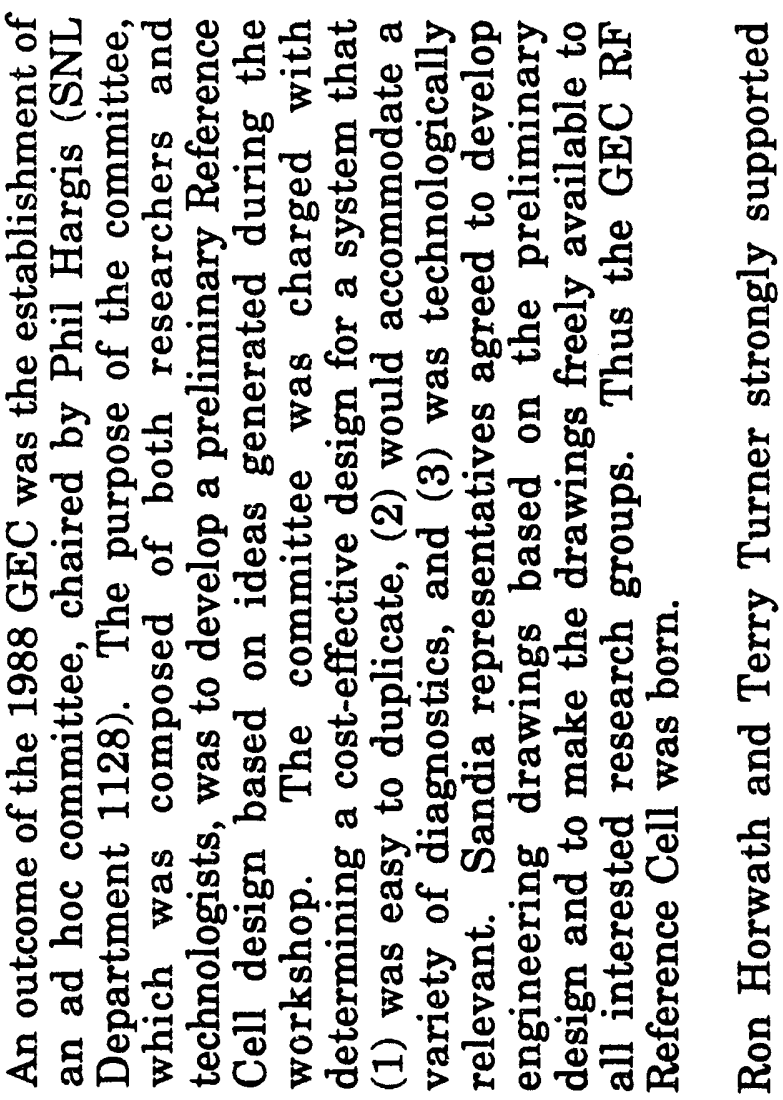



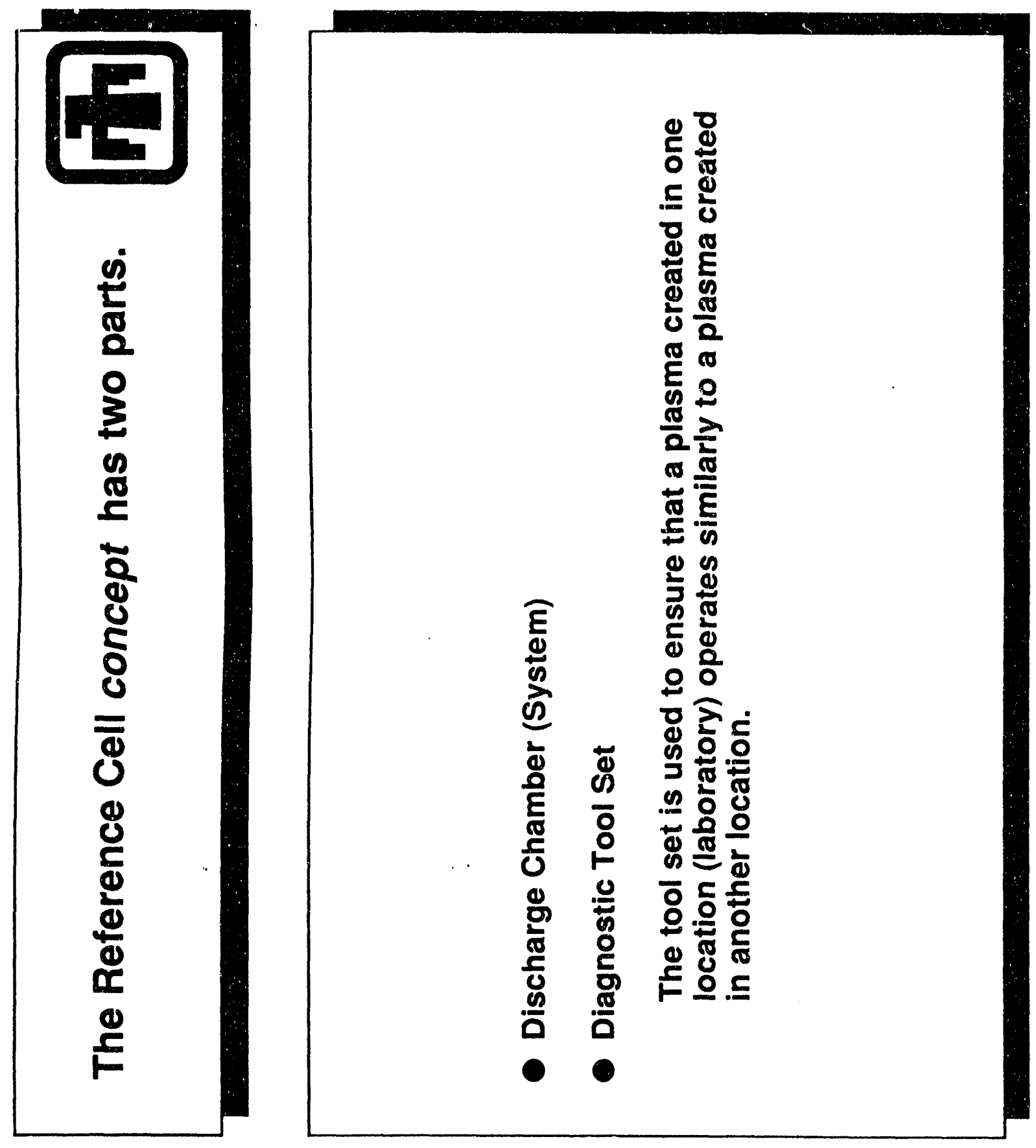


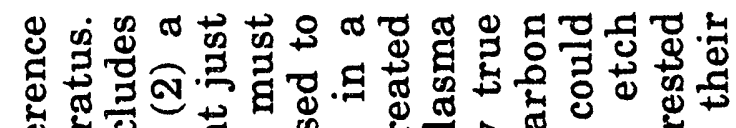

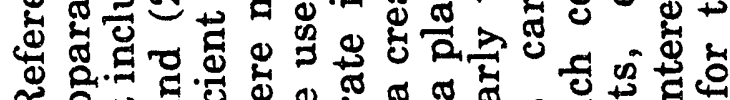

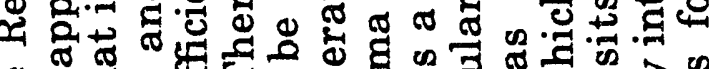
ه

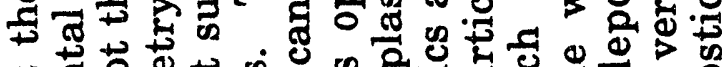

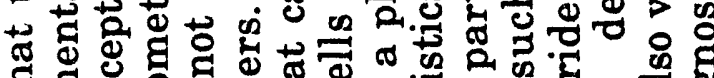
¿

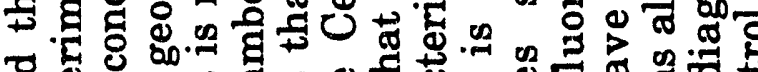
ర0 ثै ¿

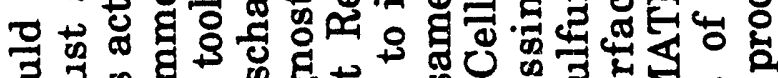

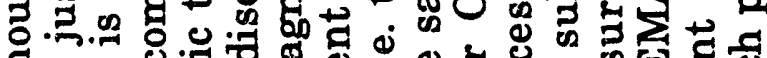

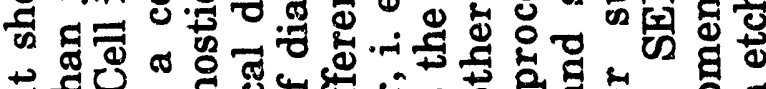

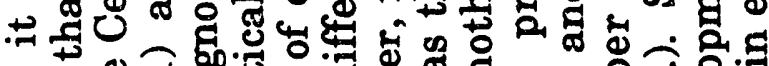

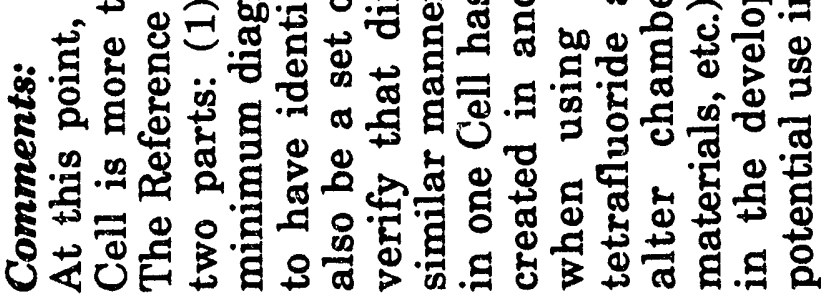




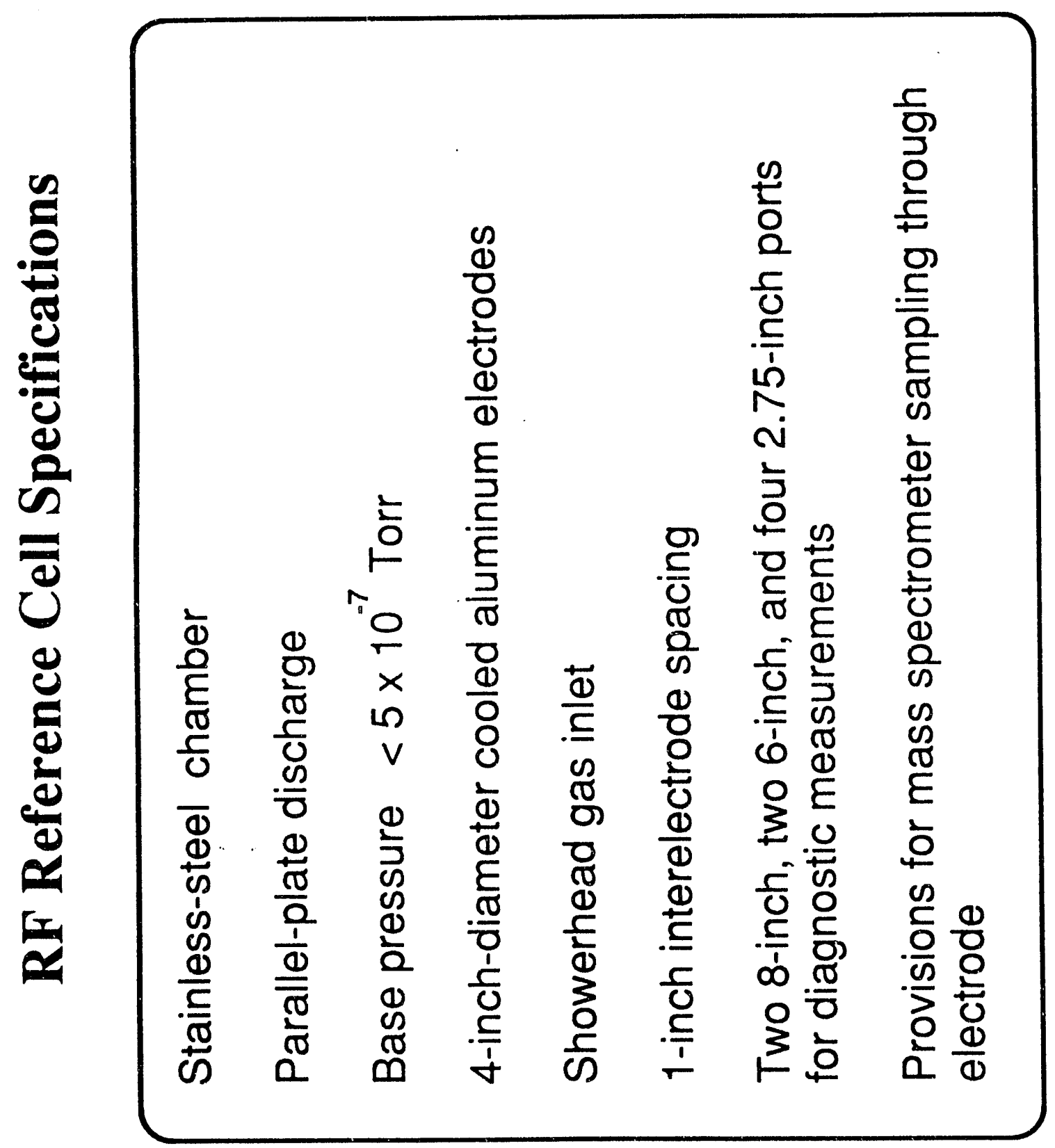




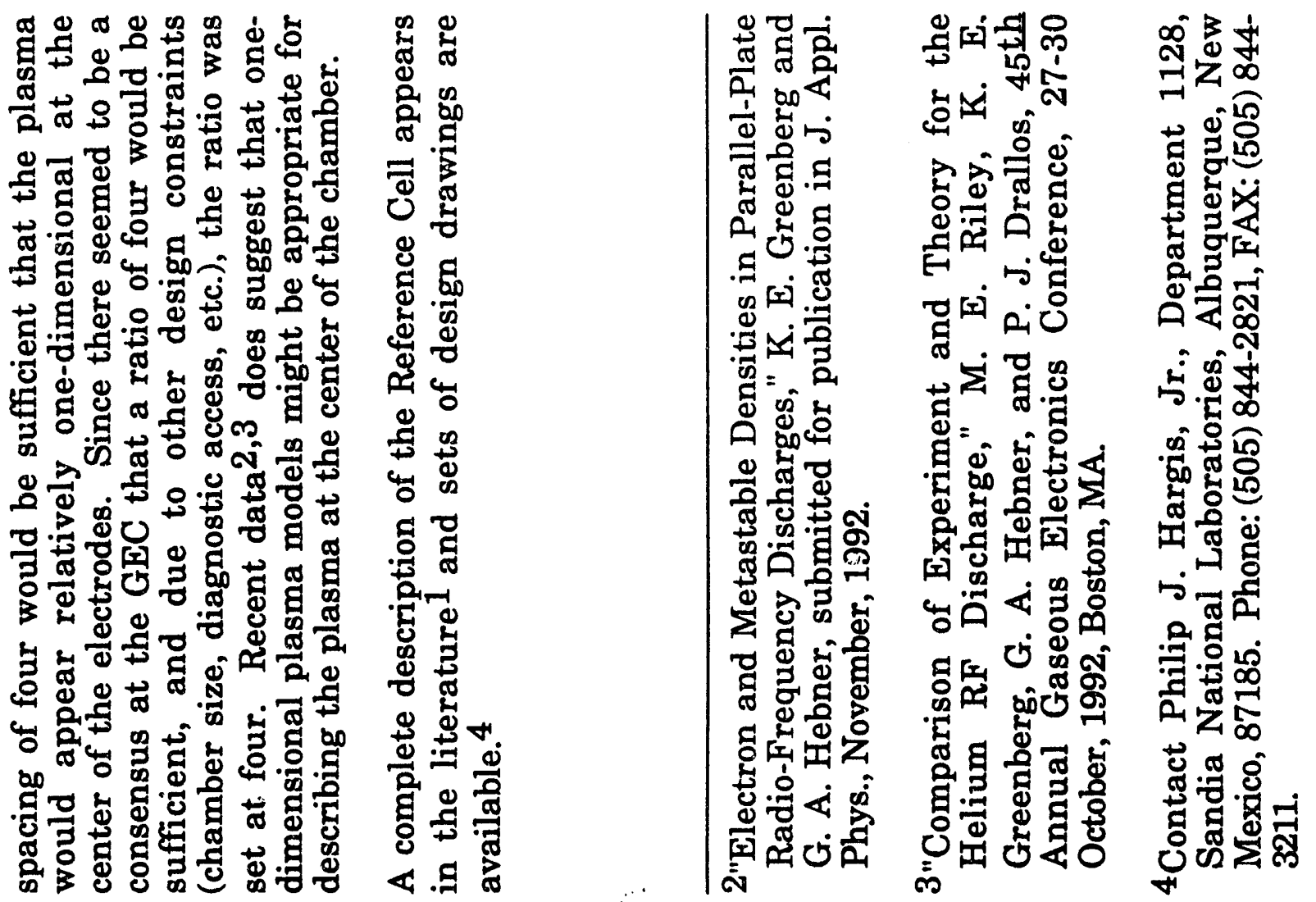

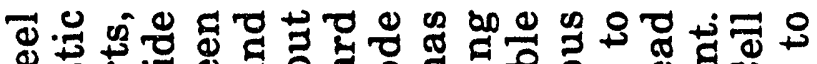
丹 क

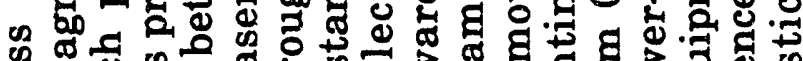

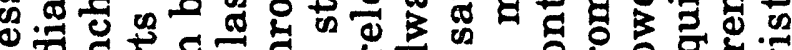

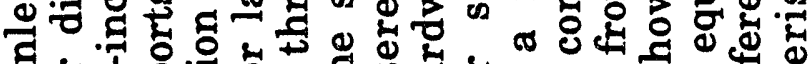

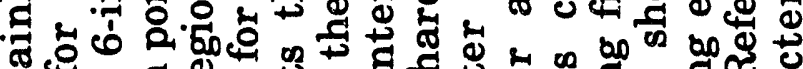
क ⿻上丨

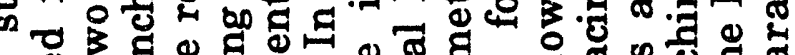
๙ $\infty$ क

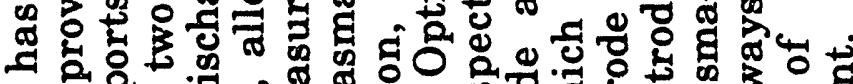

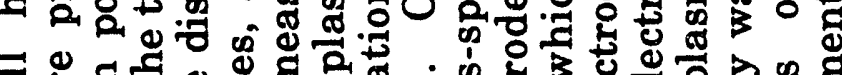

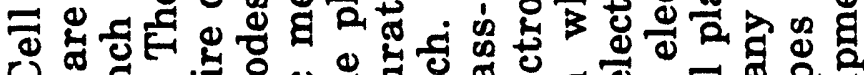
क 丹

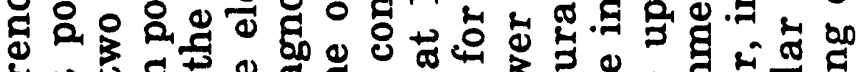
屯屯

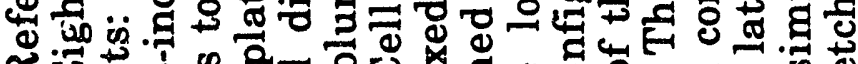

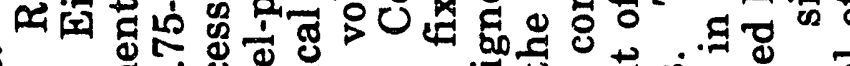

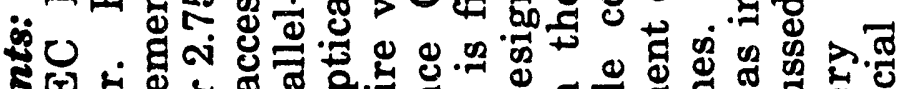

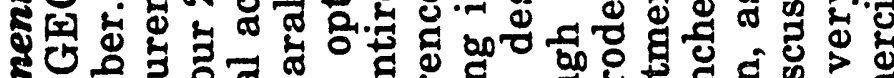

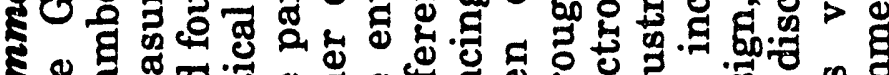

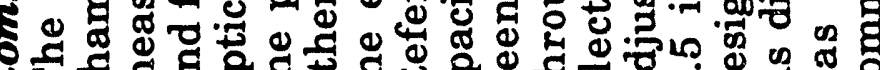
ठำ द्व

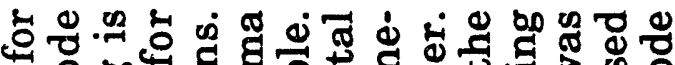

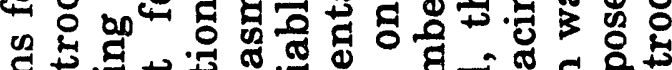

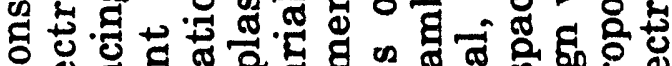

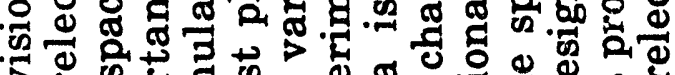

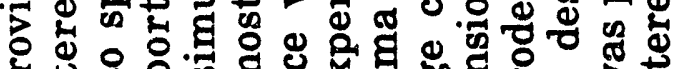

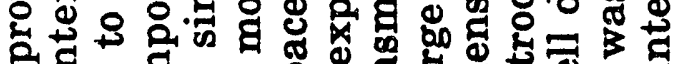
ब.

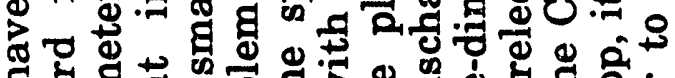

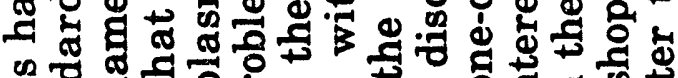

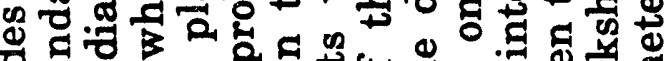

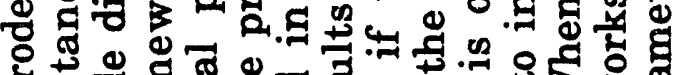

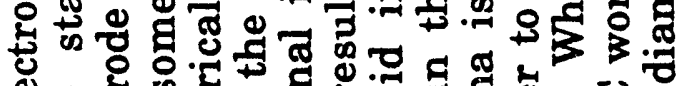

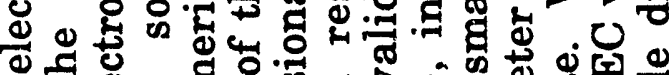


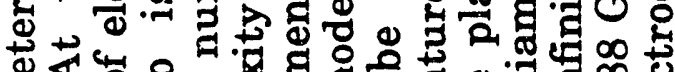

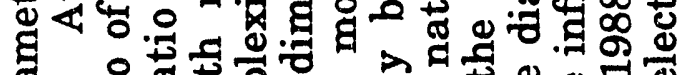

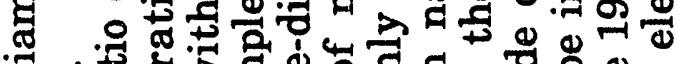
诂

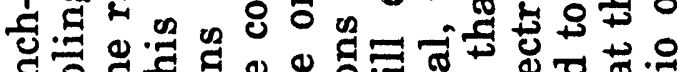

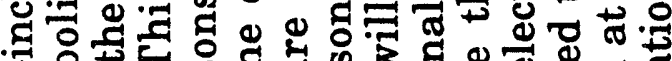

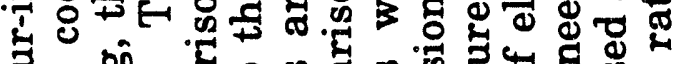

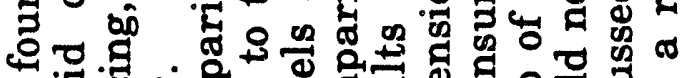
๑ 引

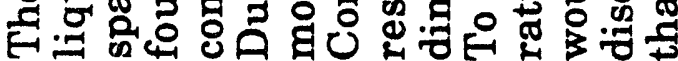




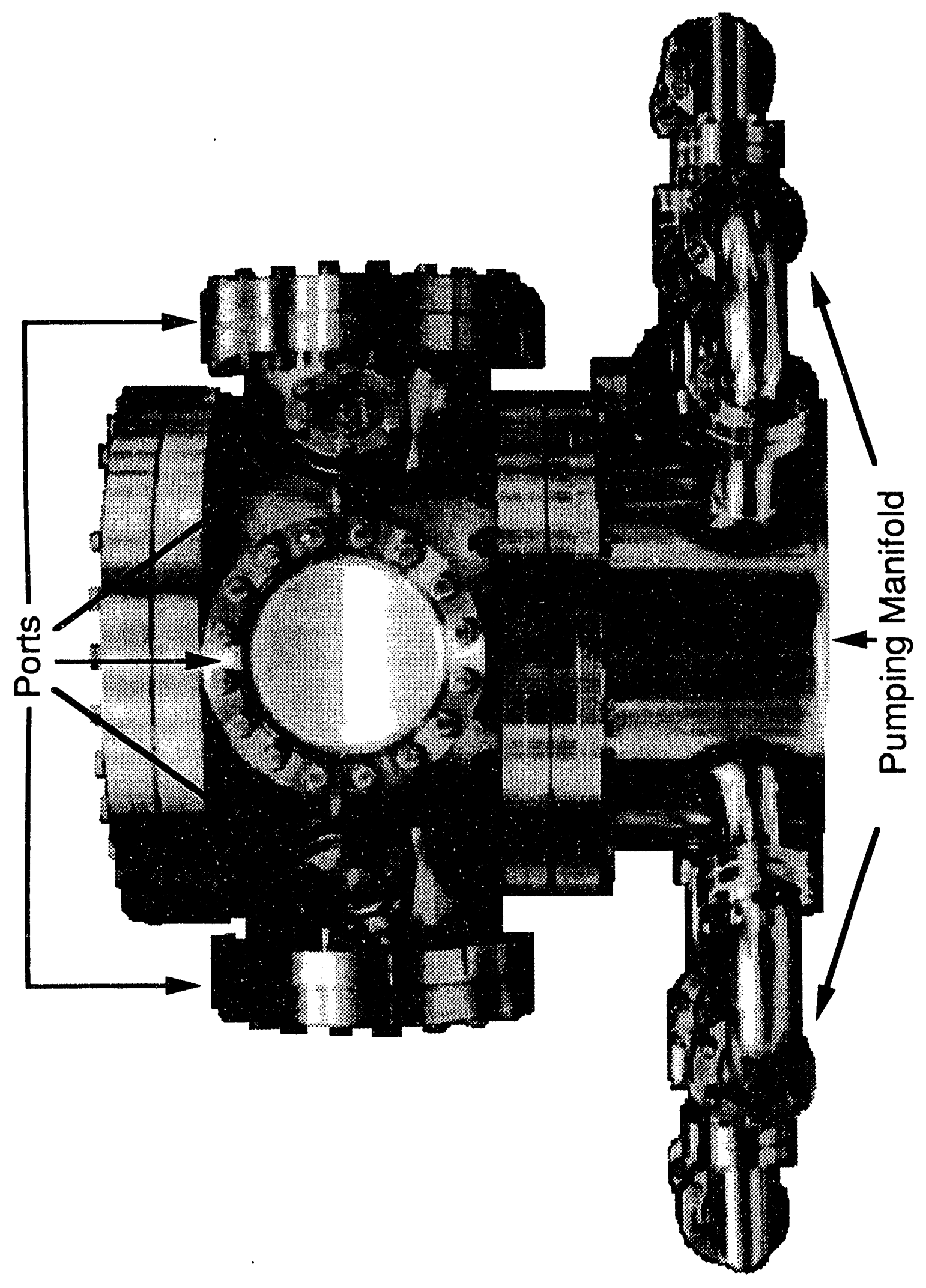




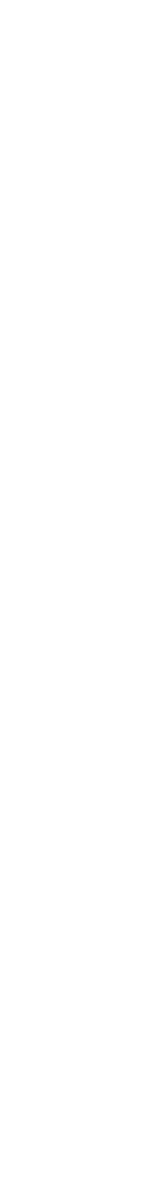




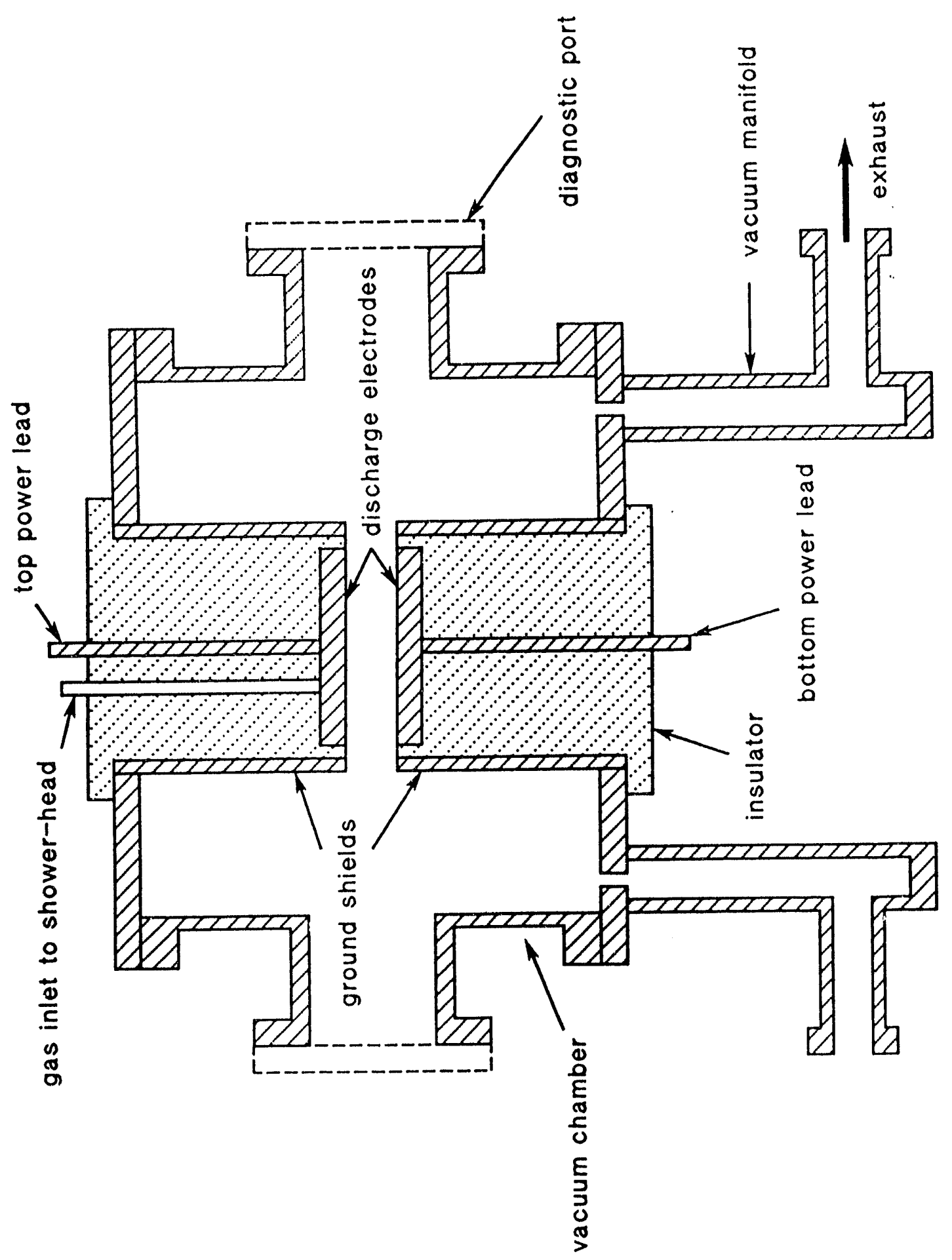




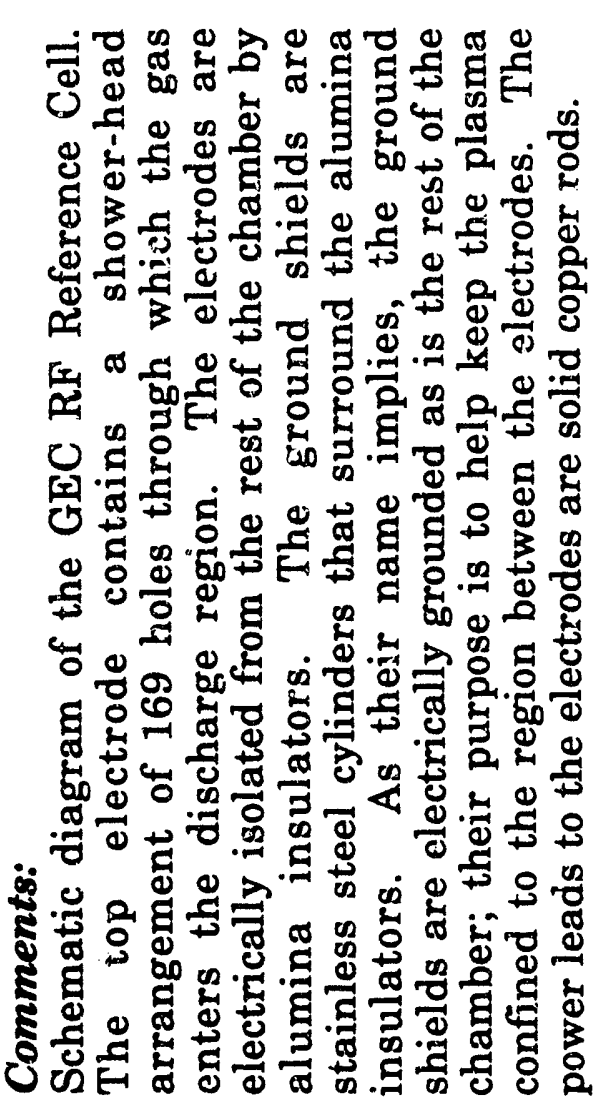




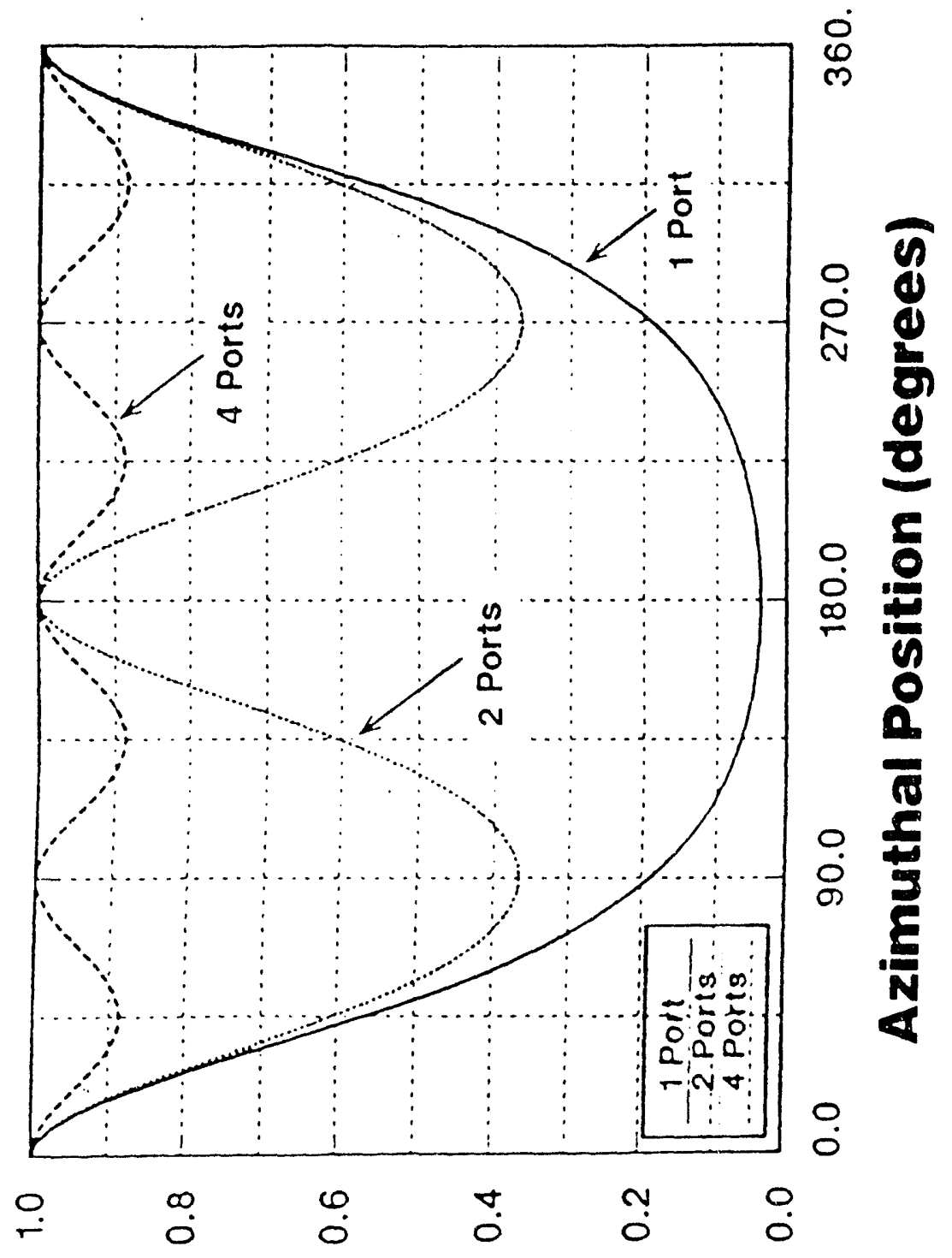

paods bu!dund on!pejoy 


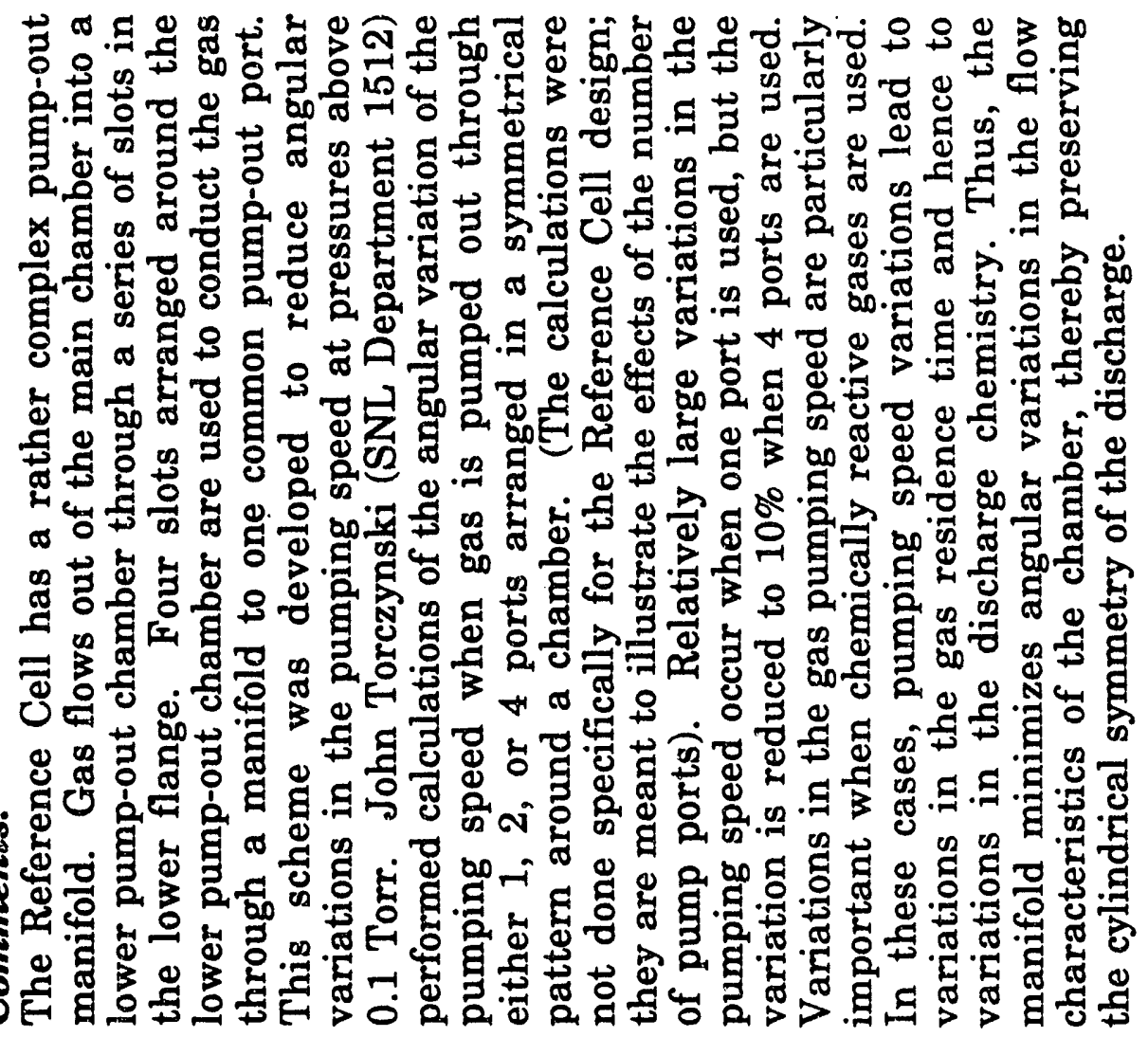



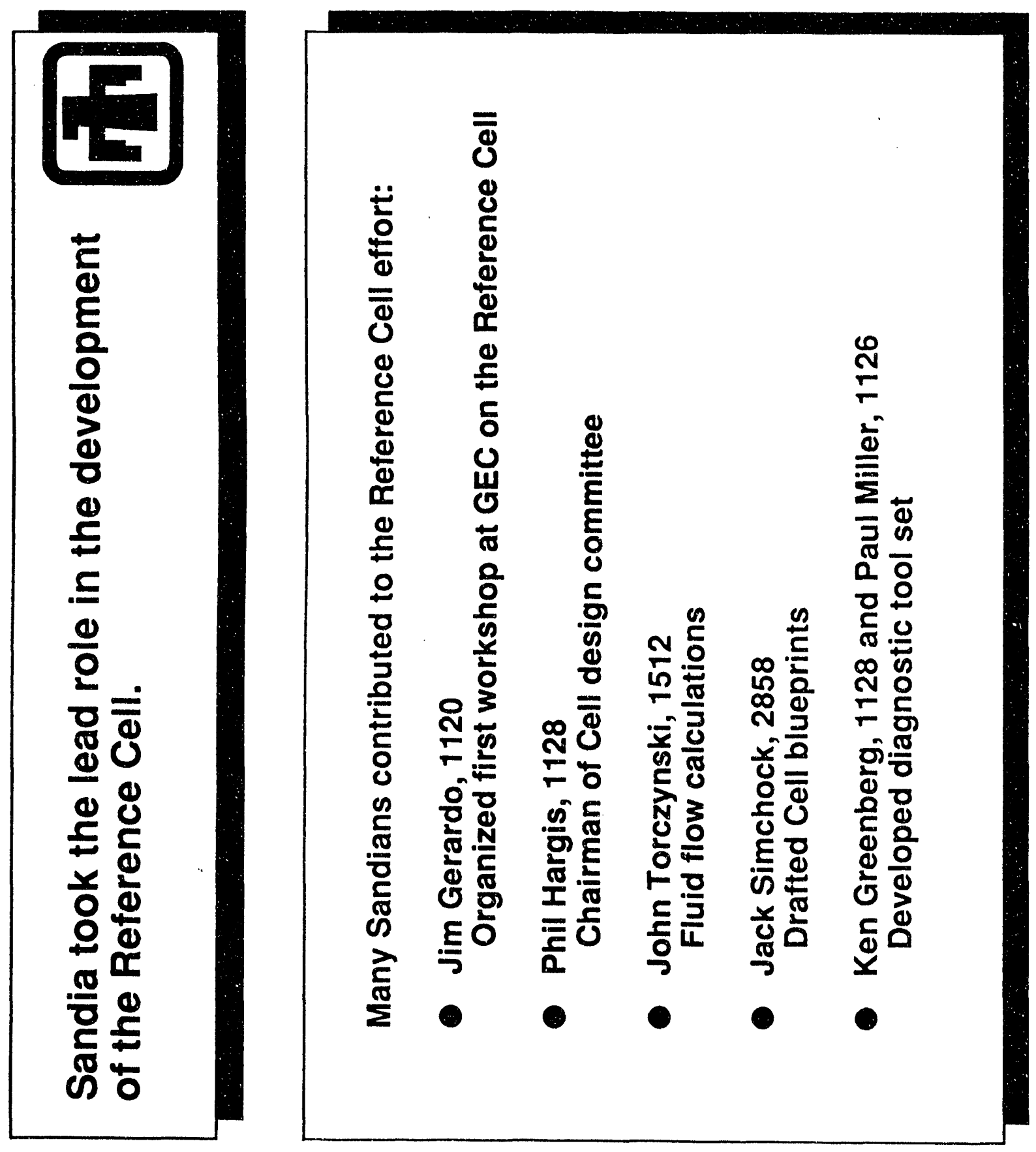


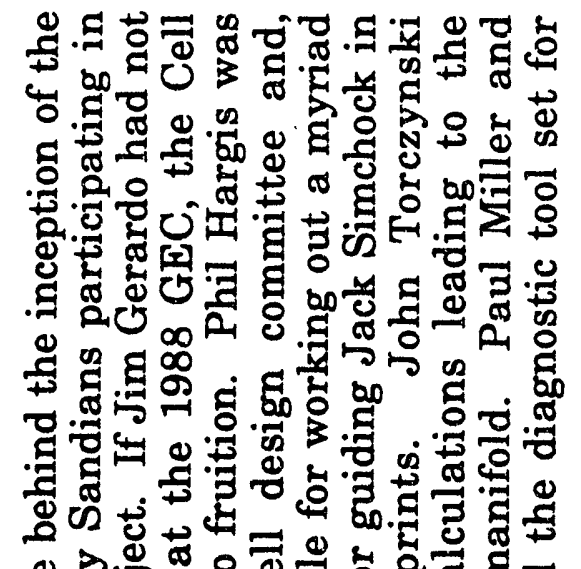
\&

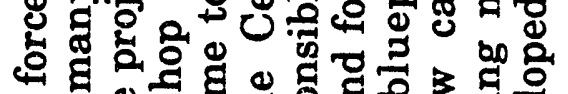

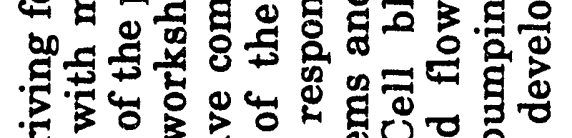

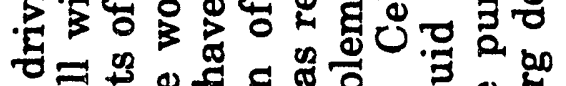

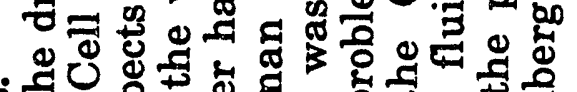

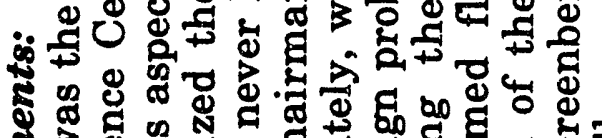

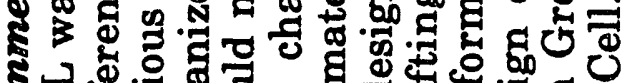

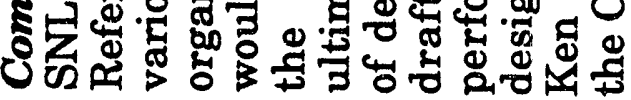

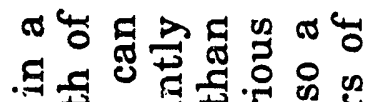

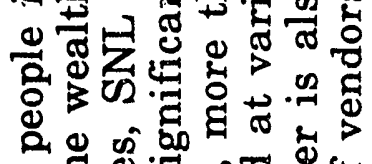

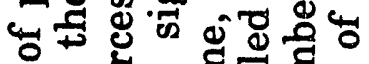

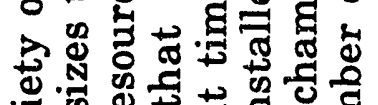

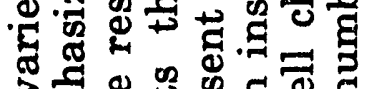

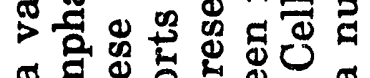
๘ ते की घ웡.

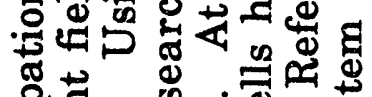

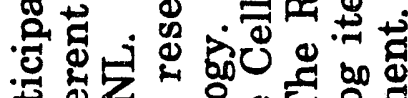
氙饮

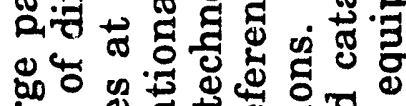
कृ 至

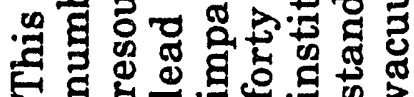




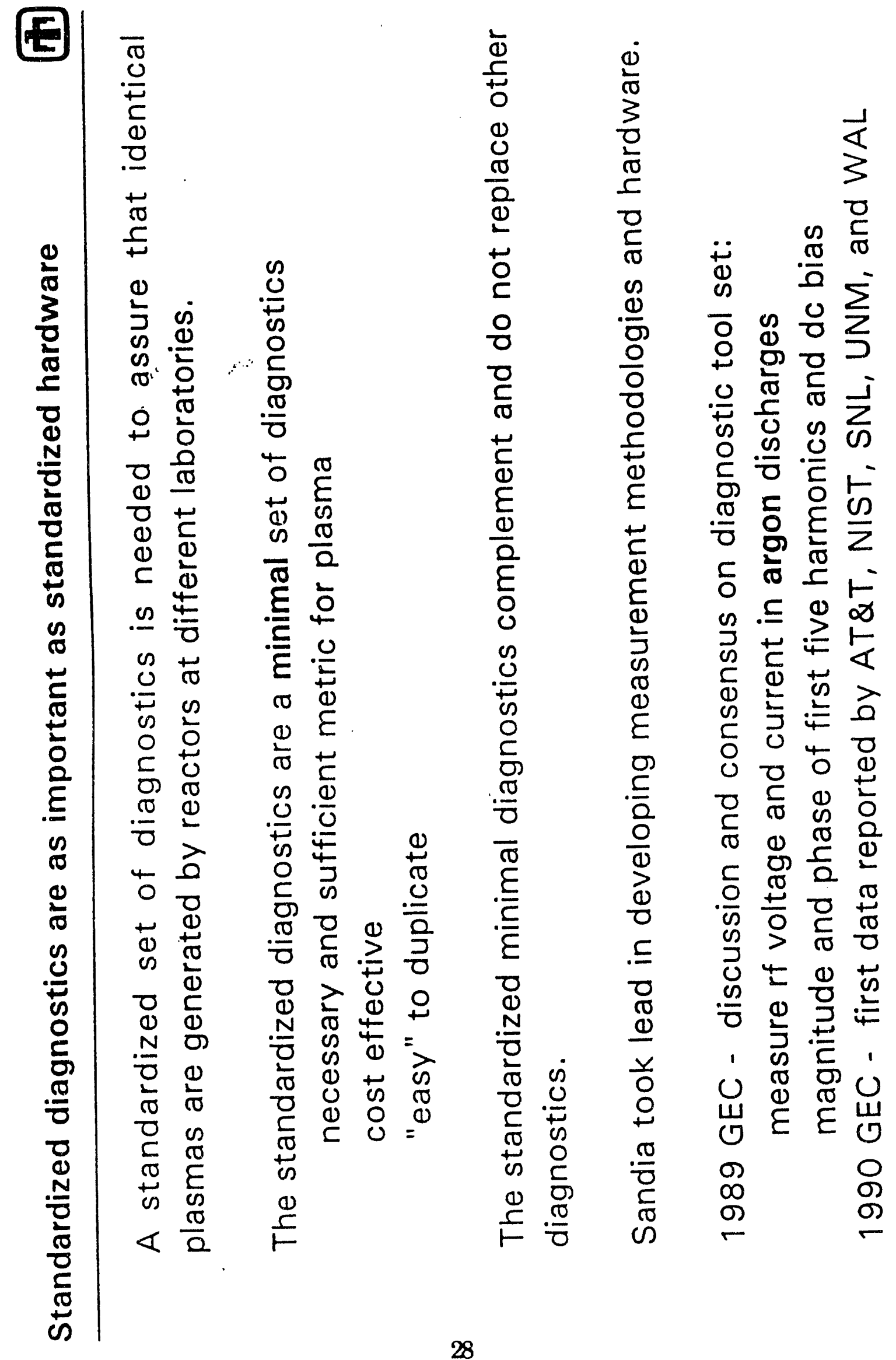




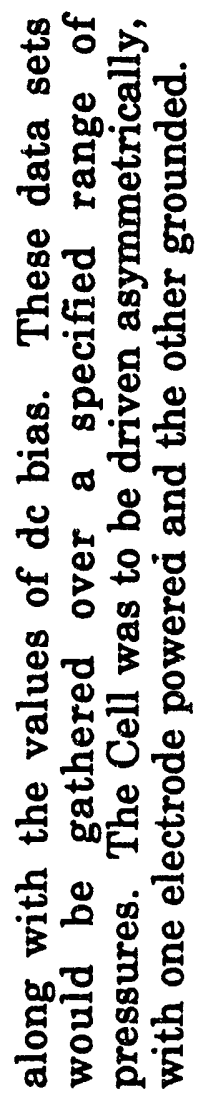

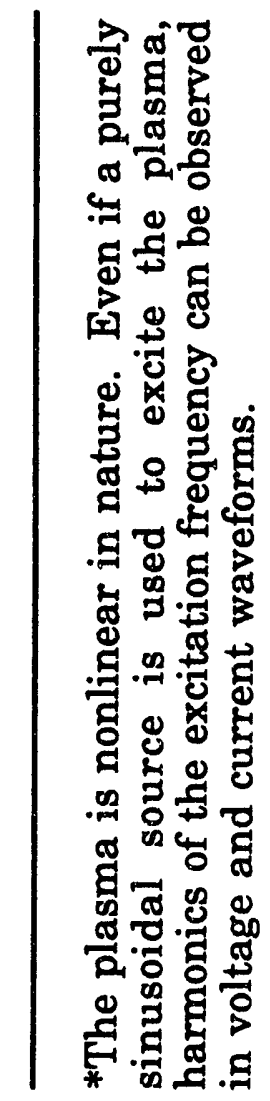

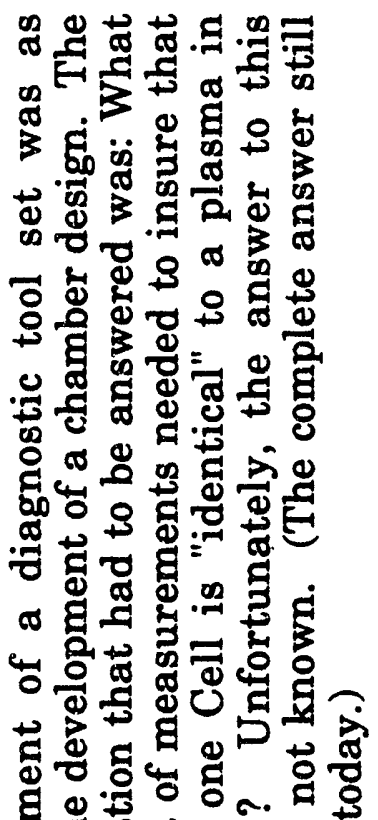

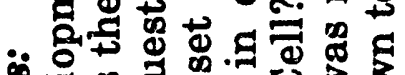

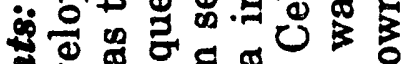

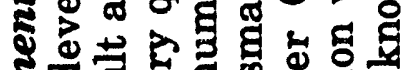

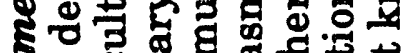

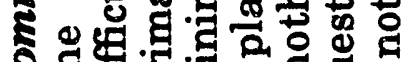

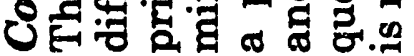

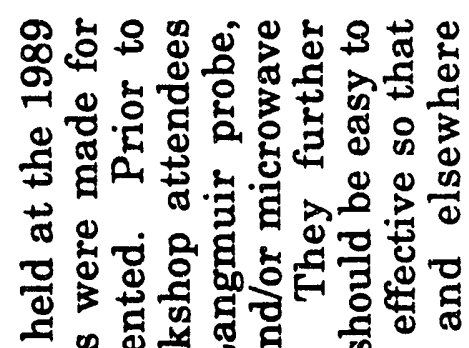

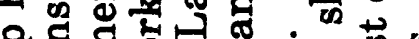

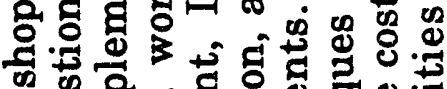

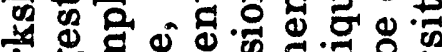
ㅇํㅇ

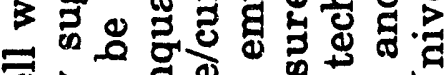

ปิ่

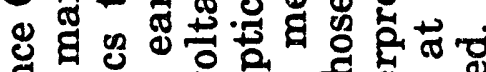

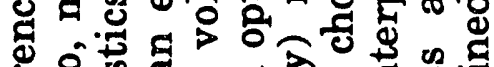

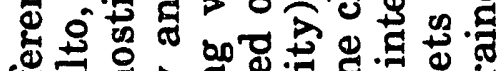

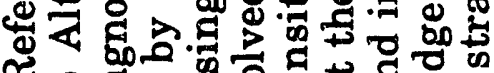
น 0 ส

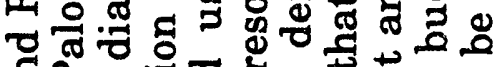

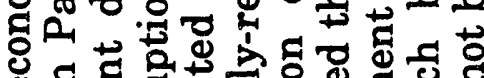

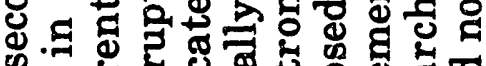
क . ○

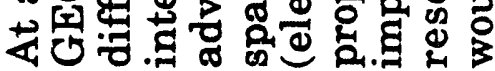

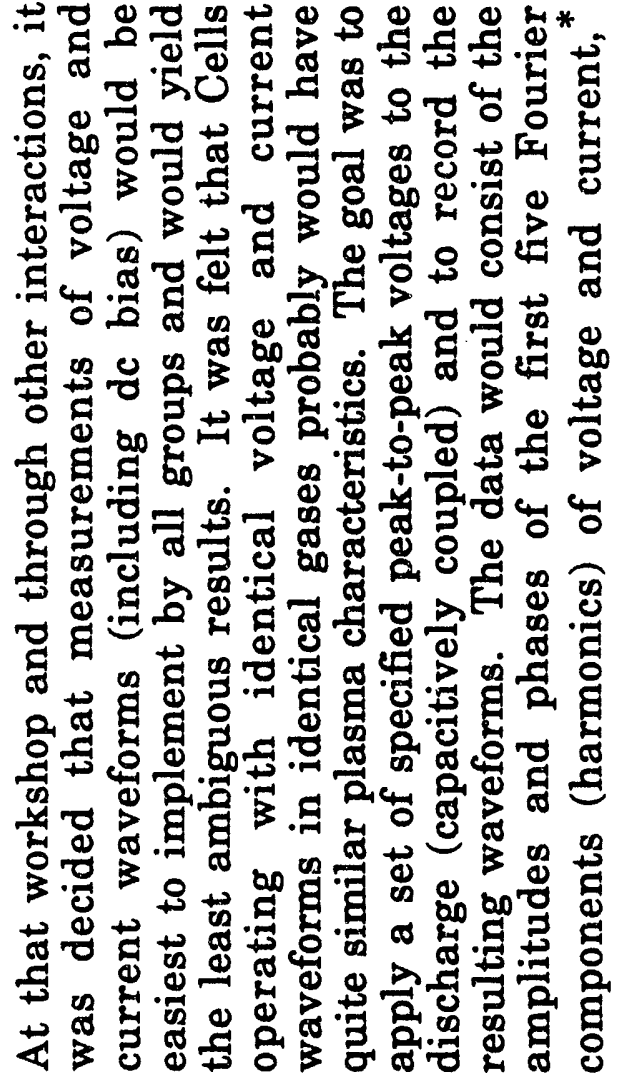



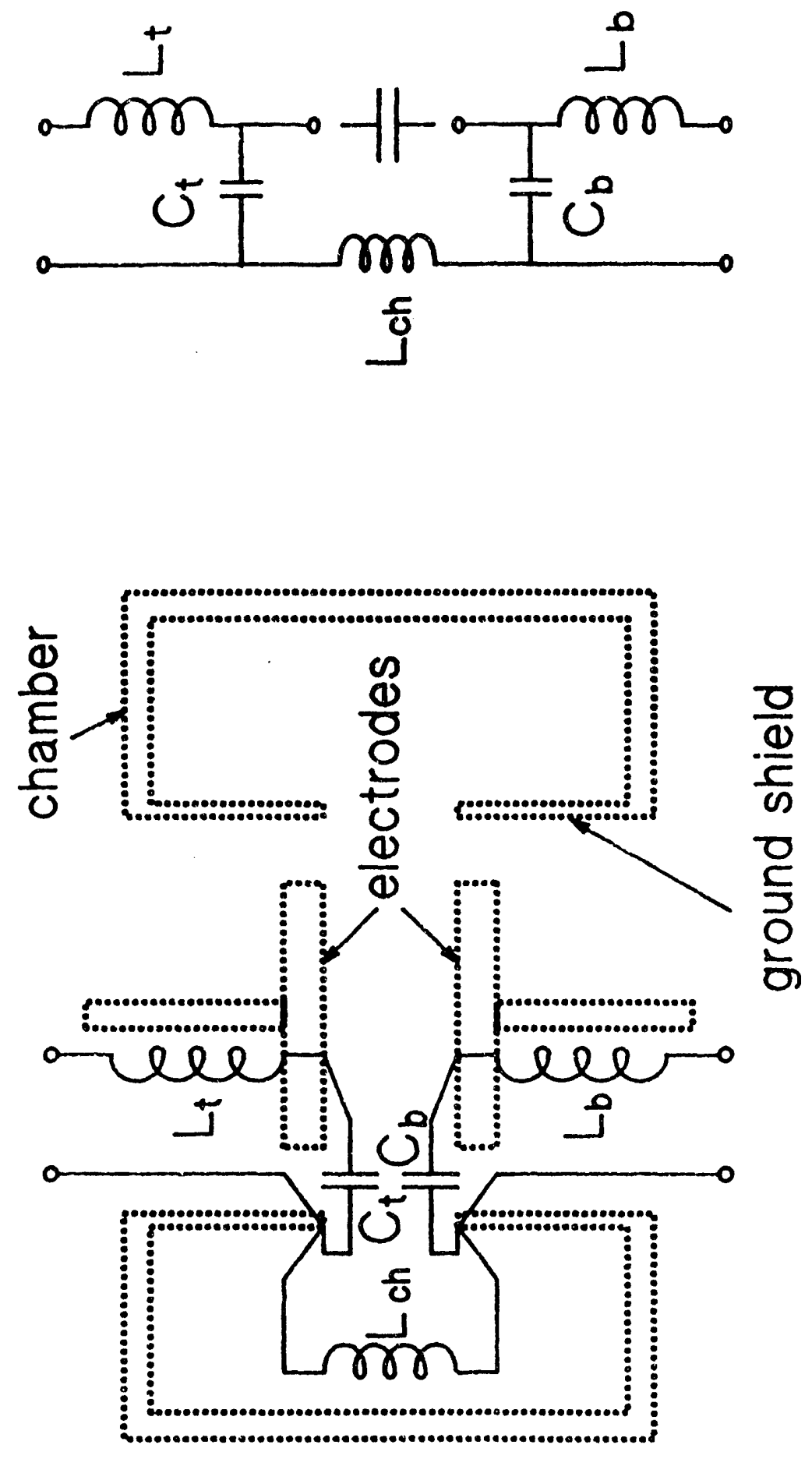


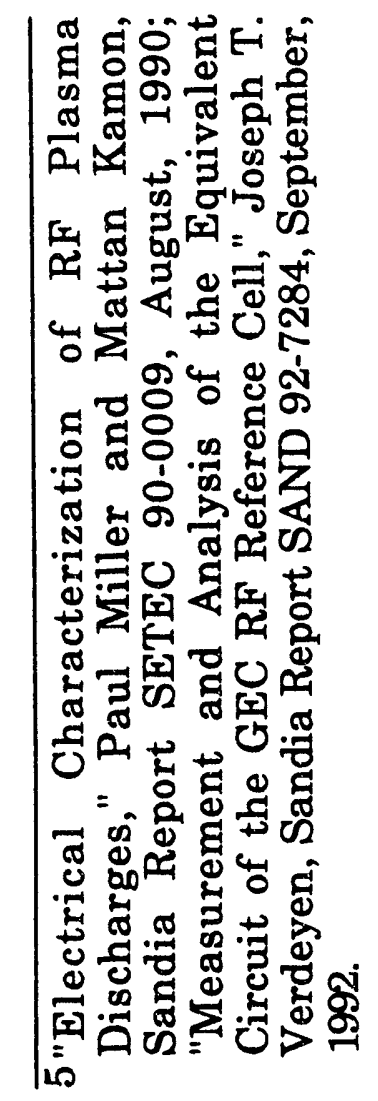

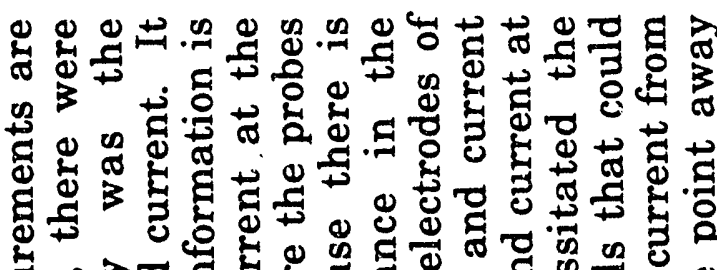

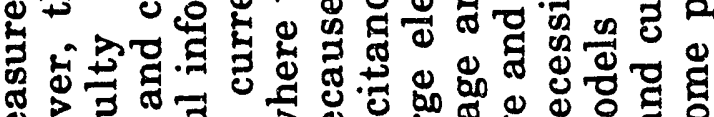
ఖँ

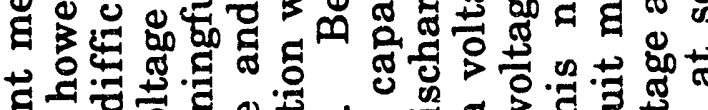
क

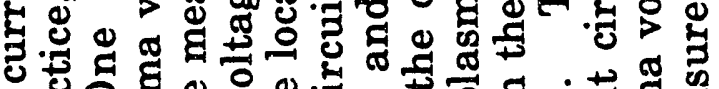
రृ ส

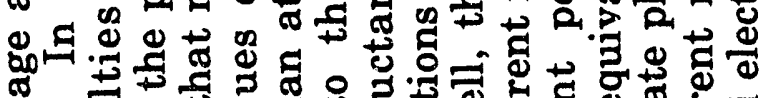

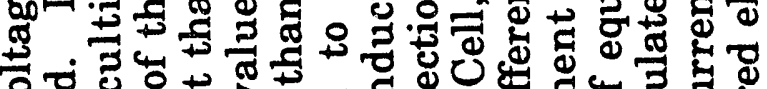
记

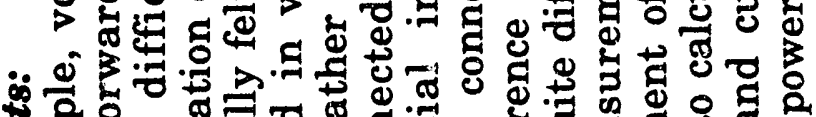

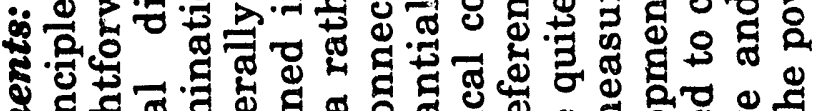
ఖ. है

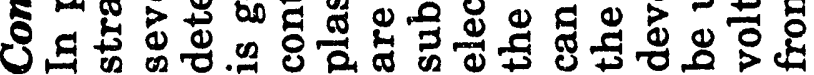

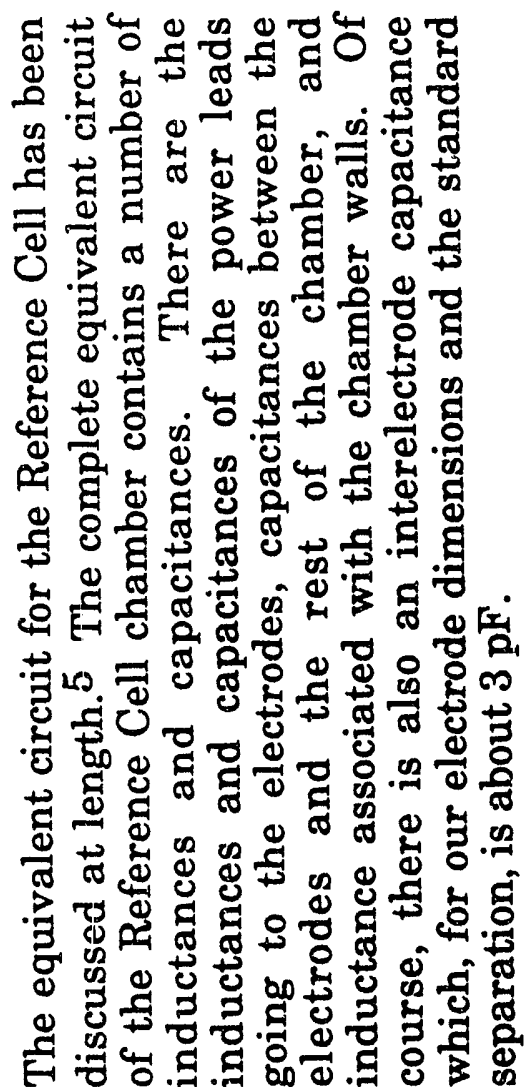




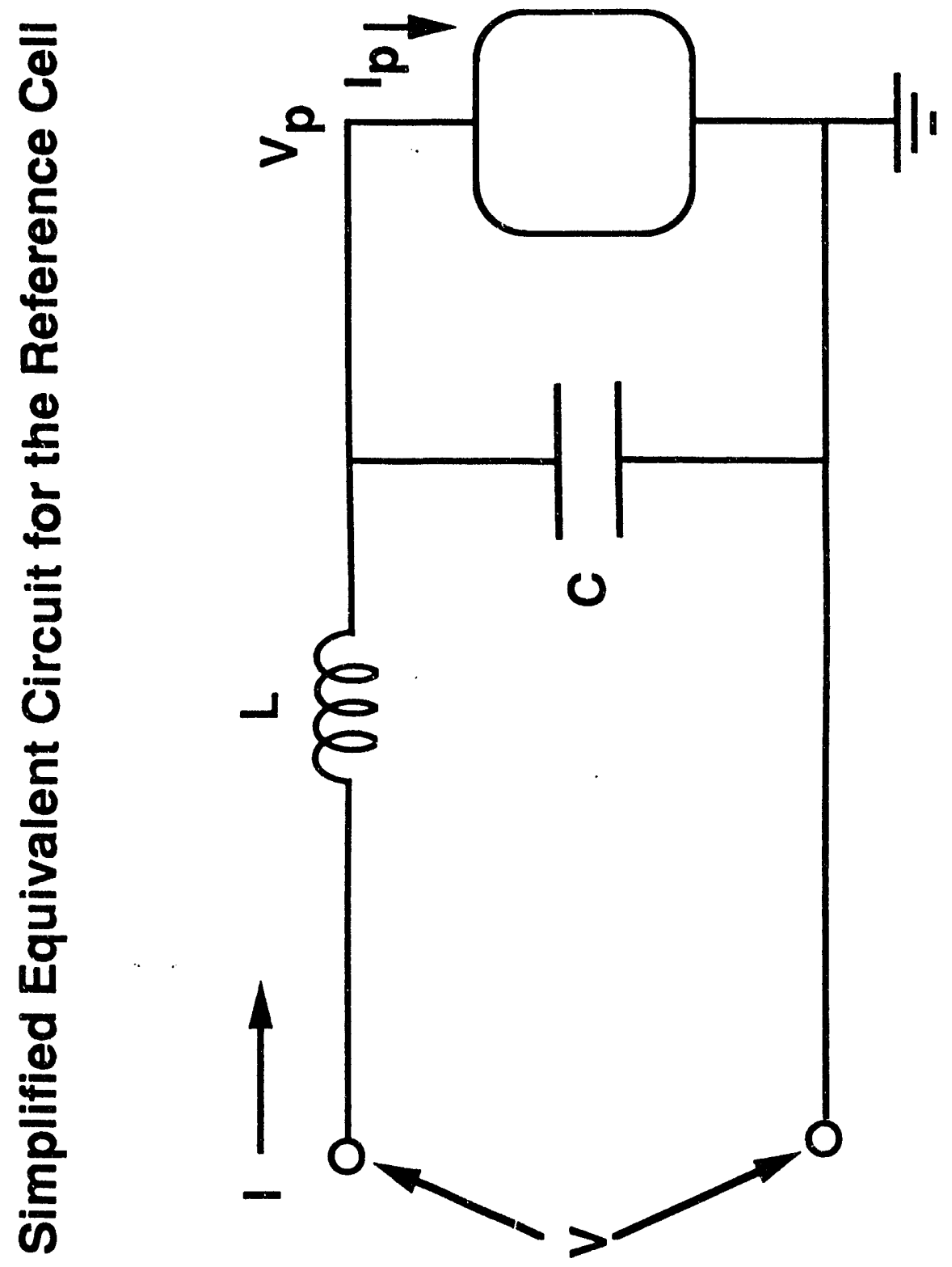



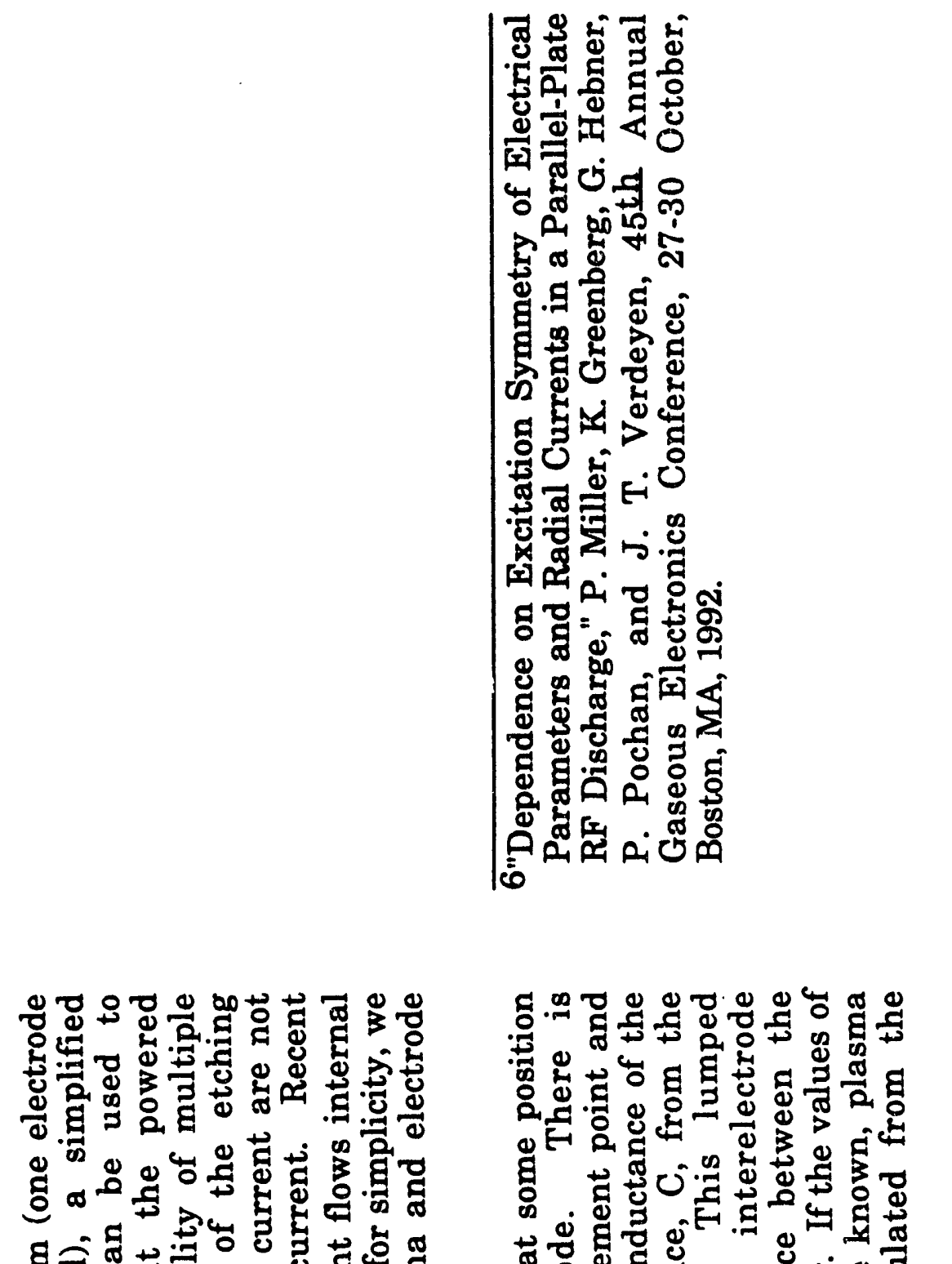

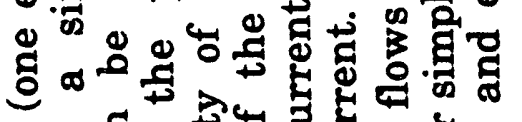
8๙

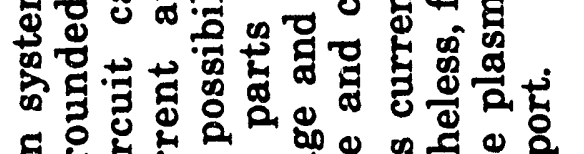

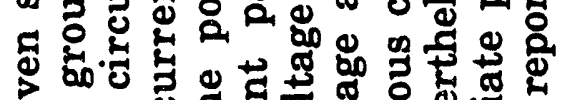

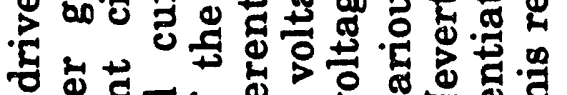

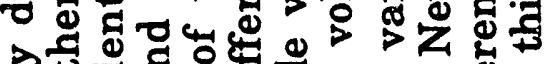
궁 क

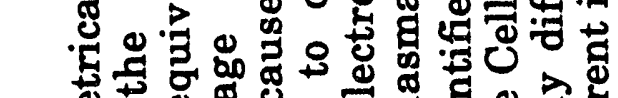

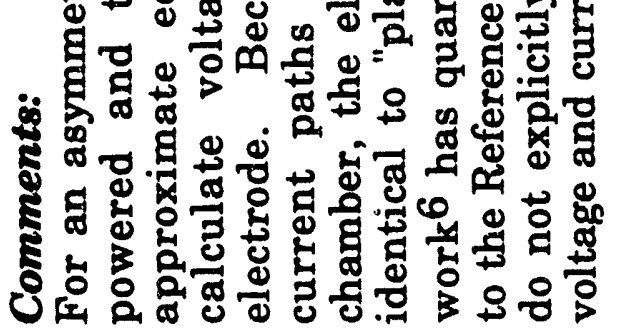

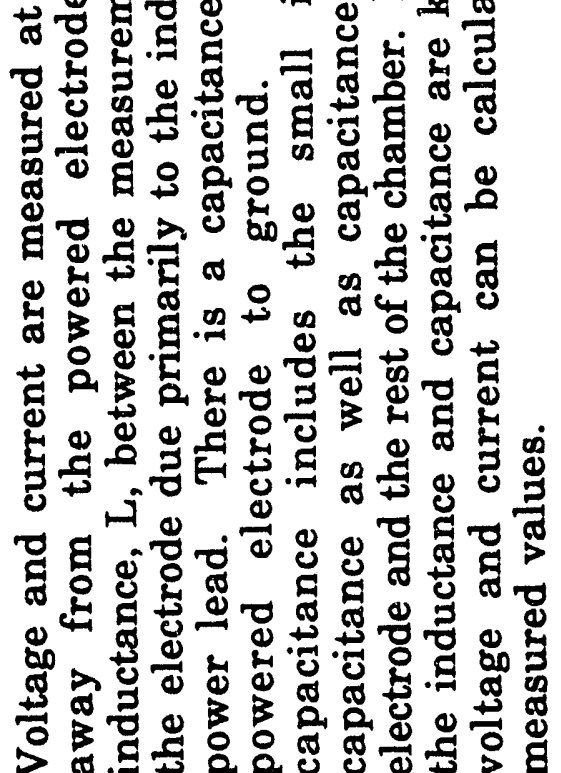
.

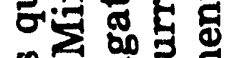

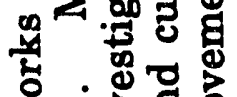

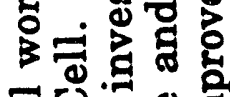
ฮั่

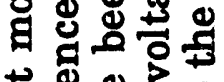
苛密蛋

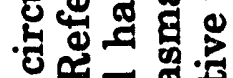

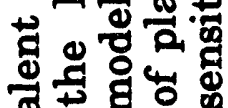
bo 0 o. 녈 영 ब.

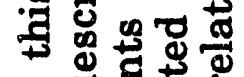

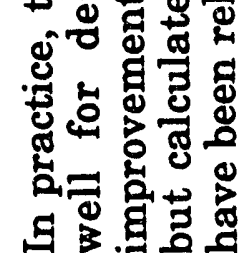



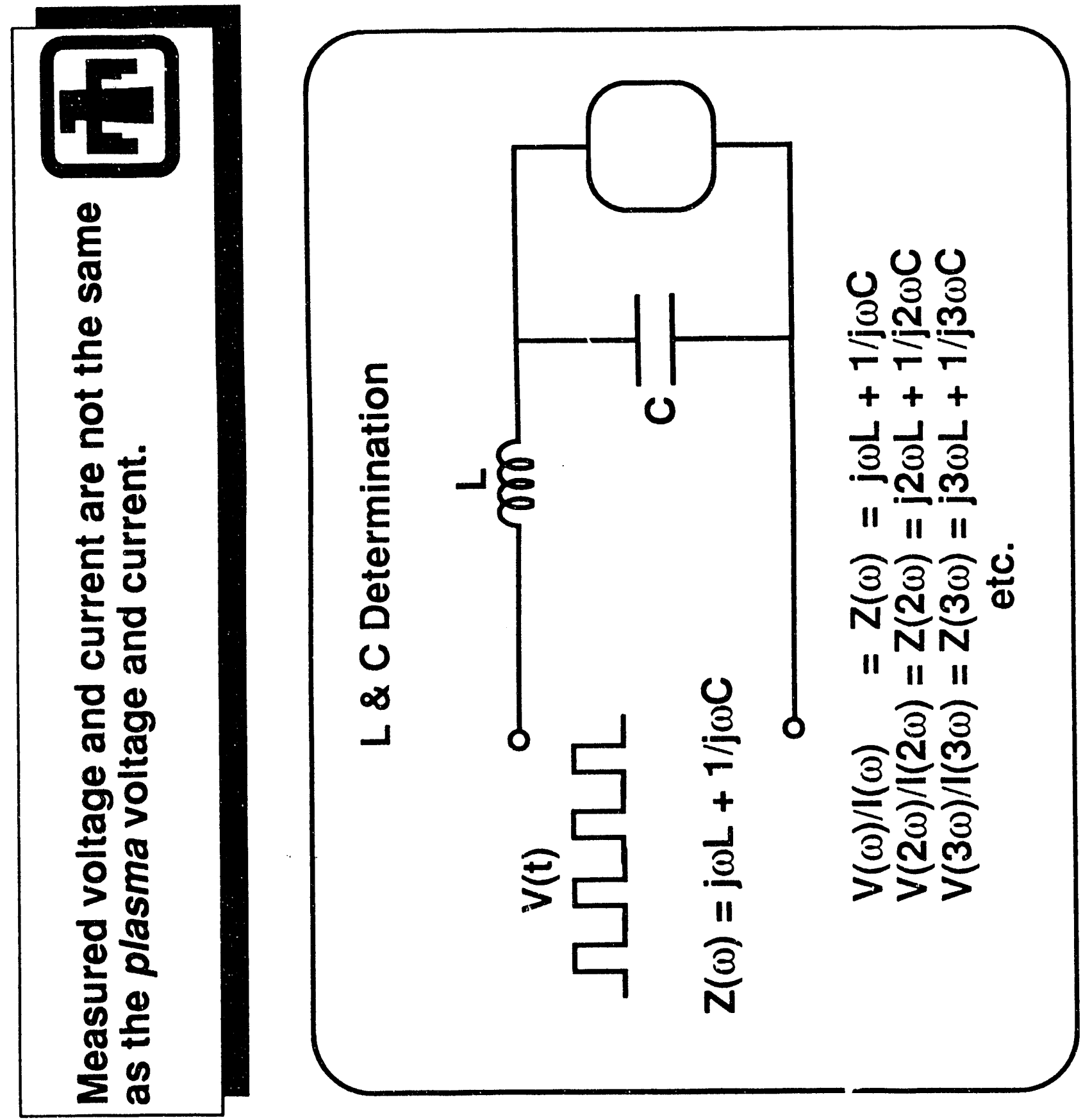

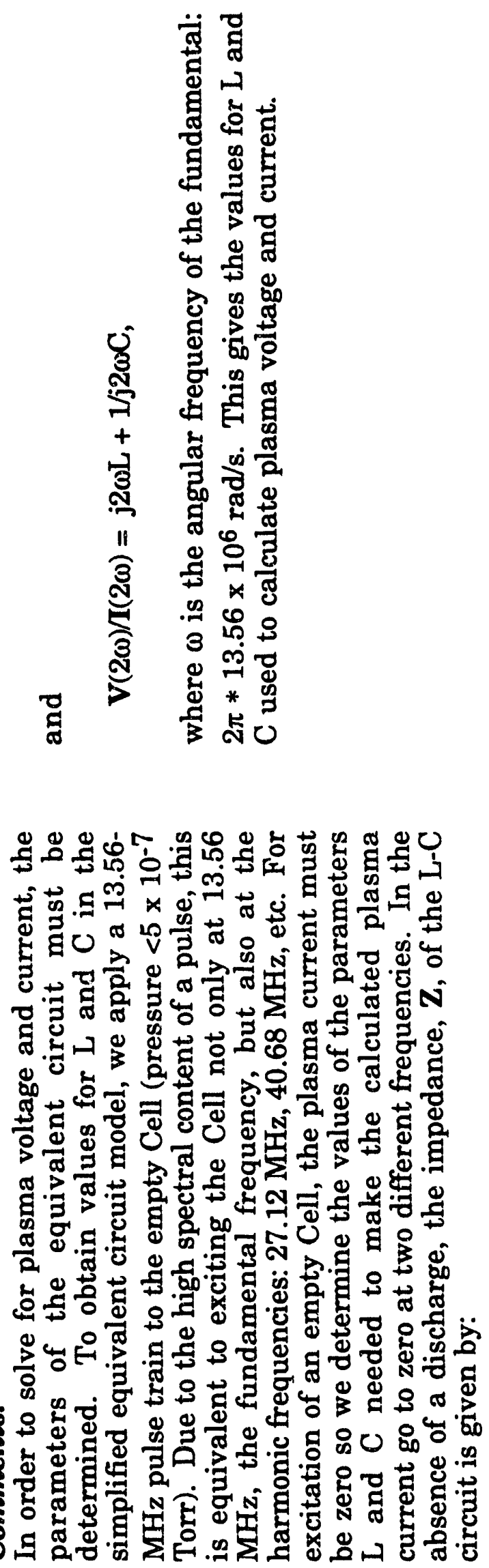

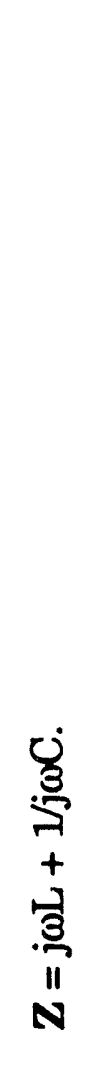

웡

๘

, 3

I.

。

了영

。

a

宁 ه.ల

응응

잉

0. 소 음

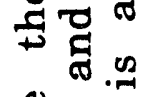

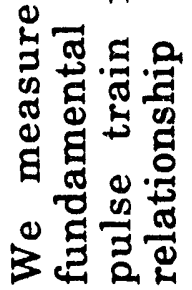

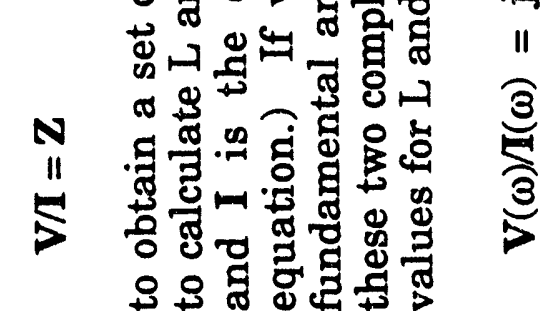




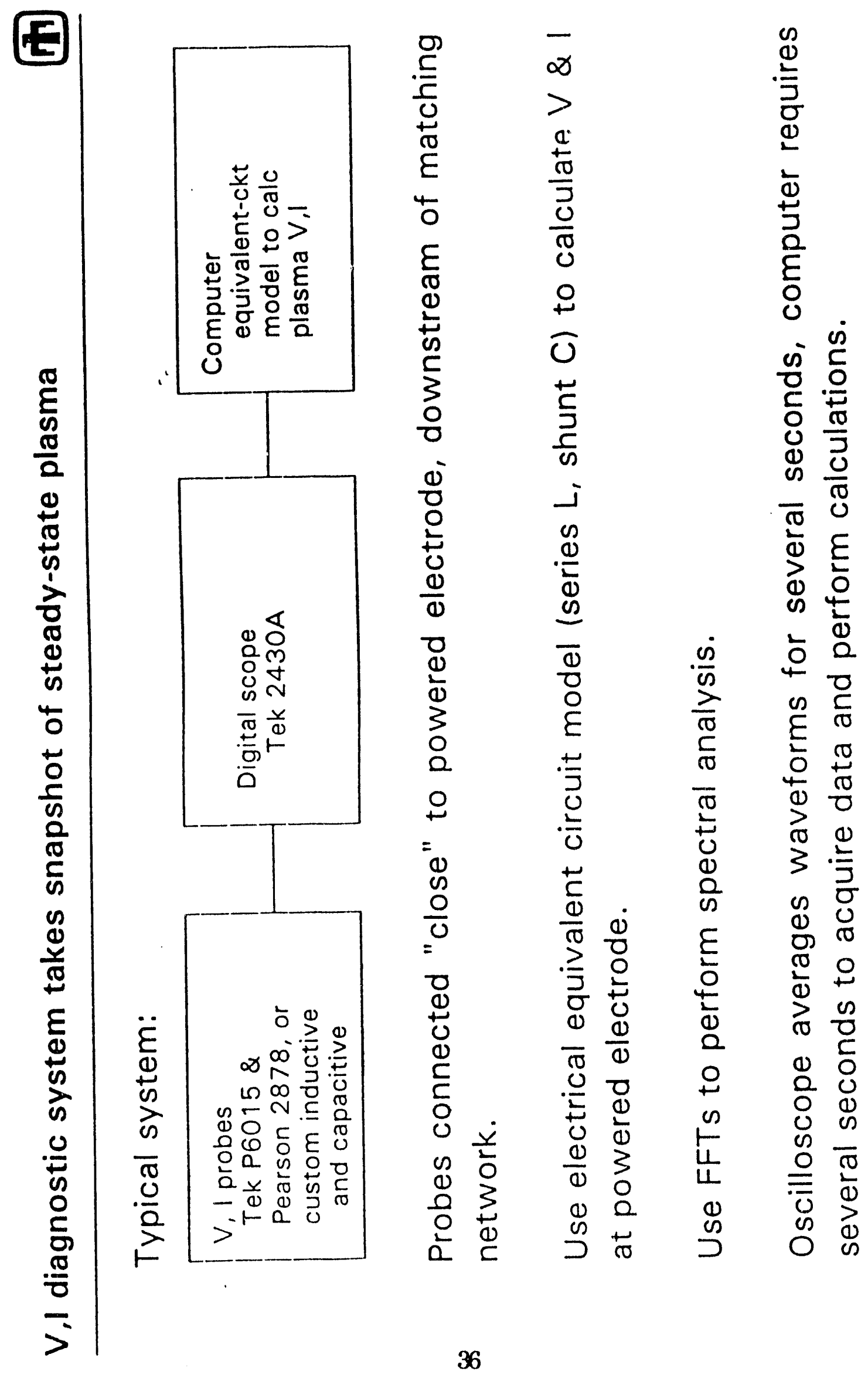




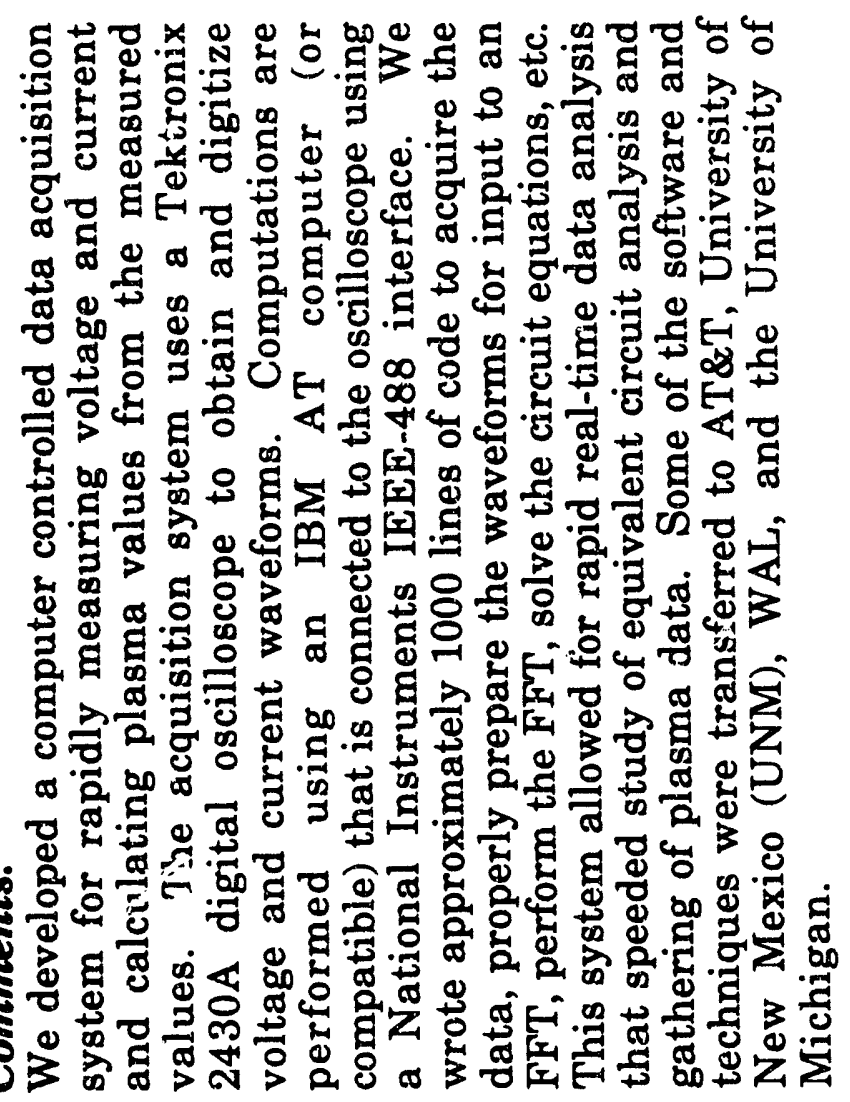




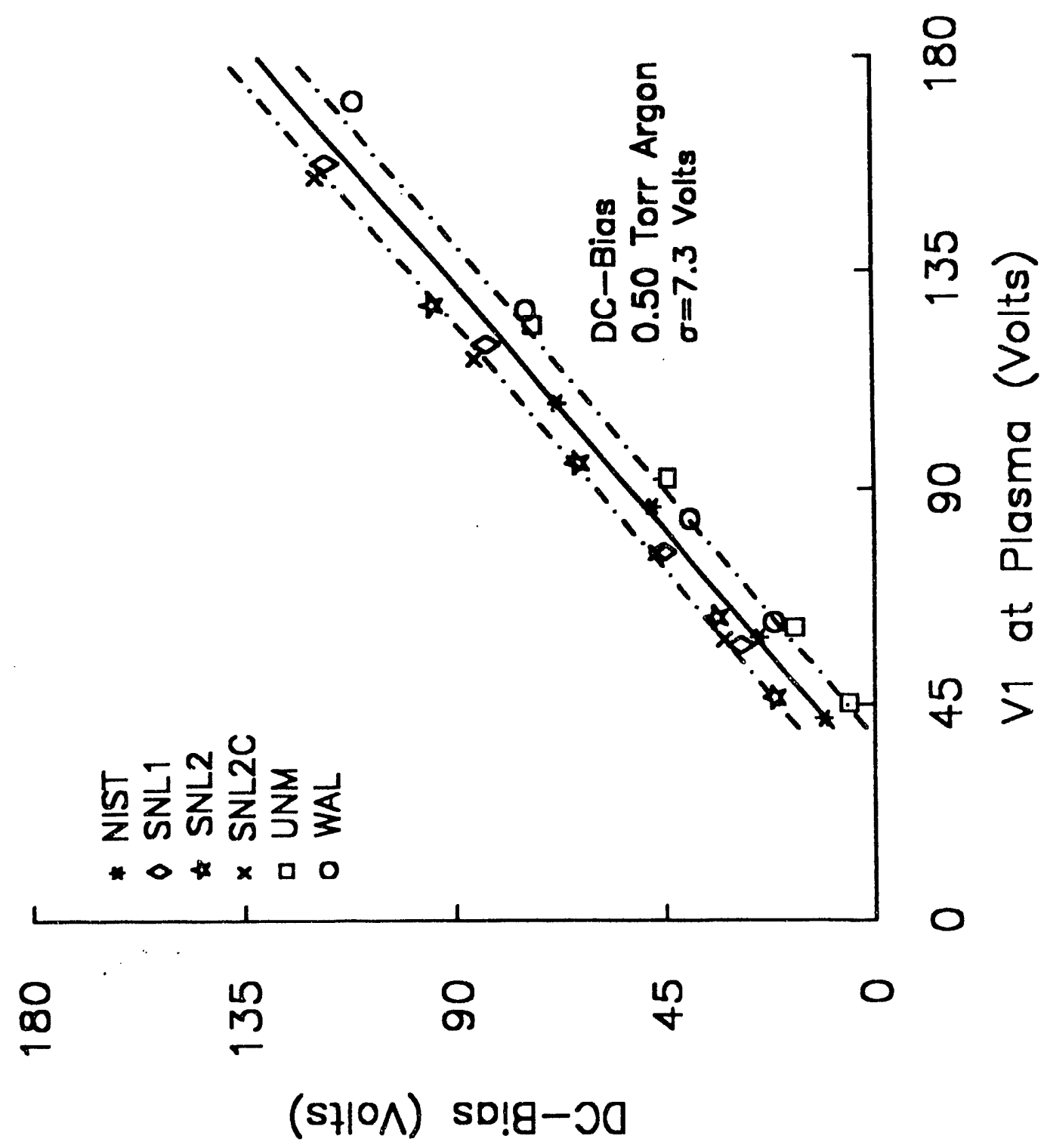




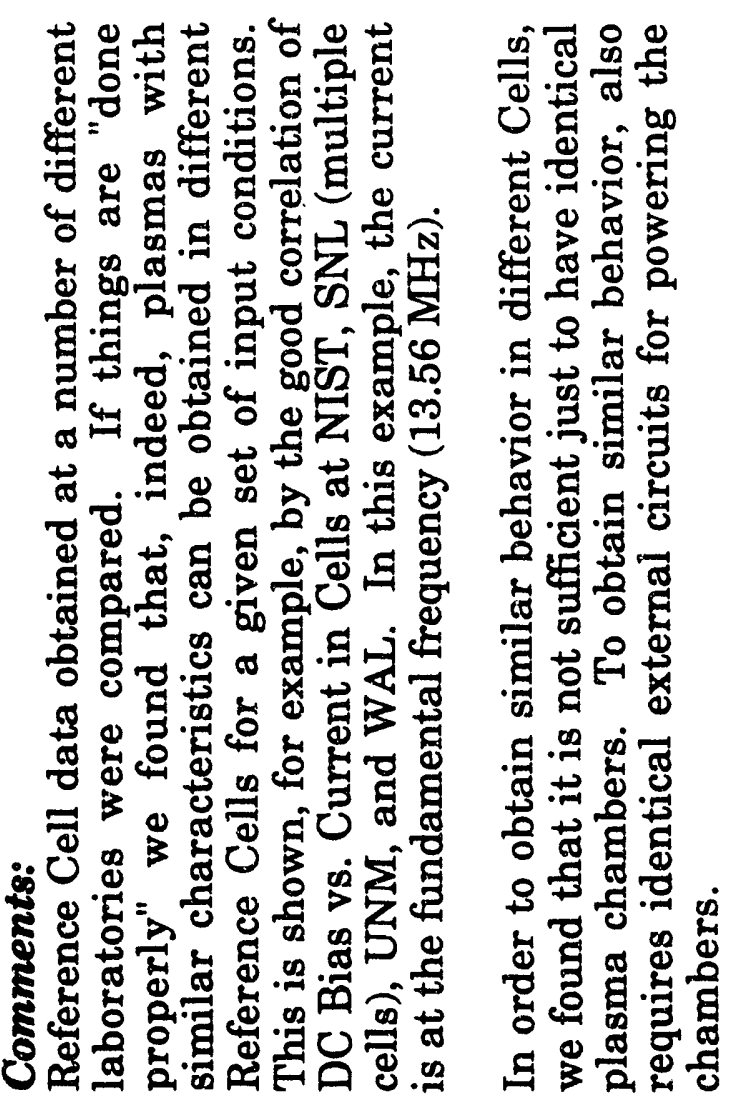




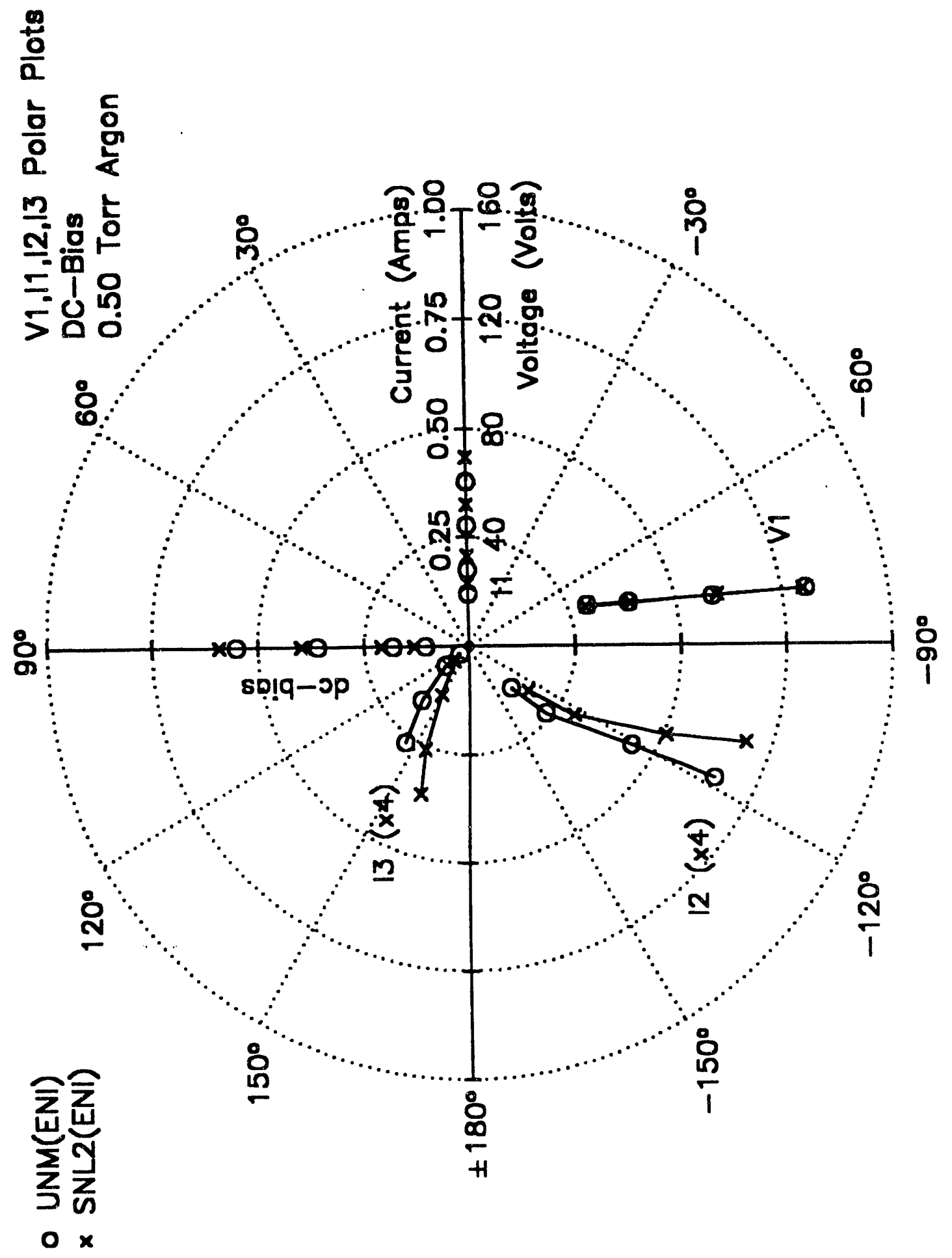




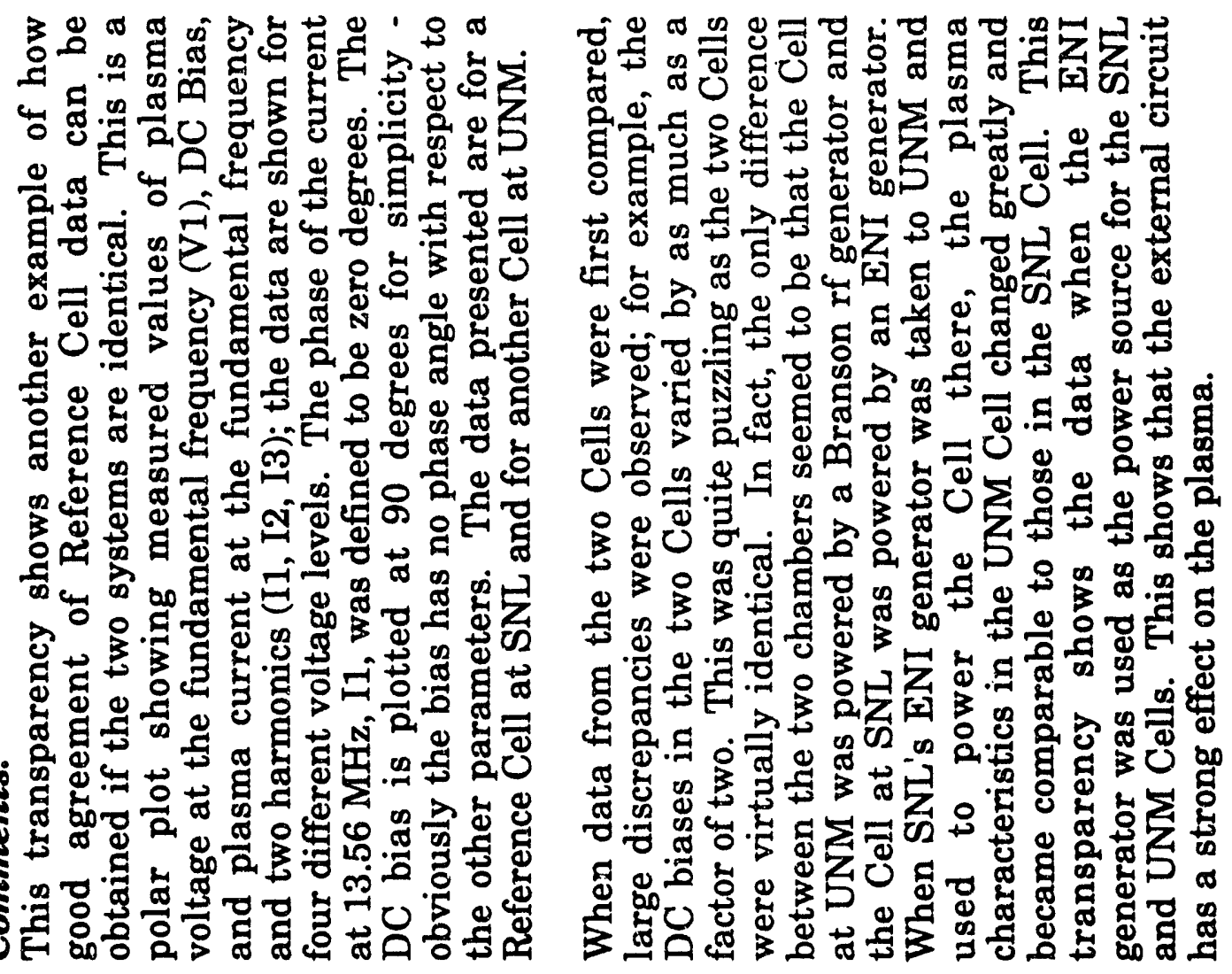




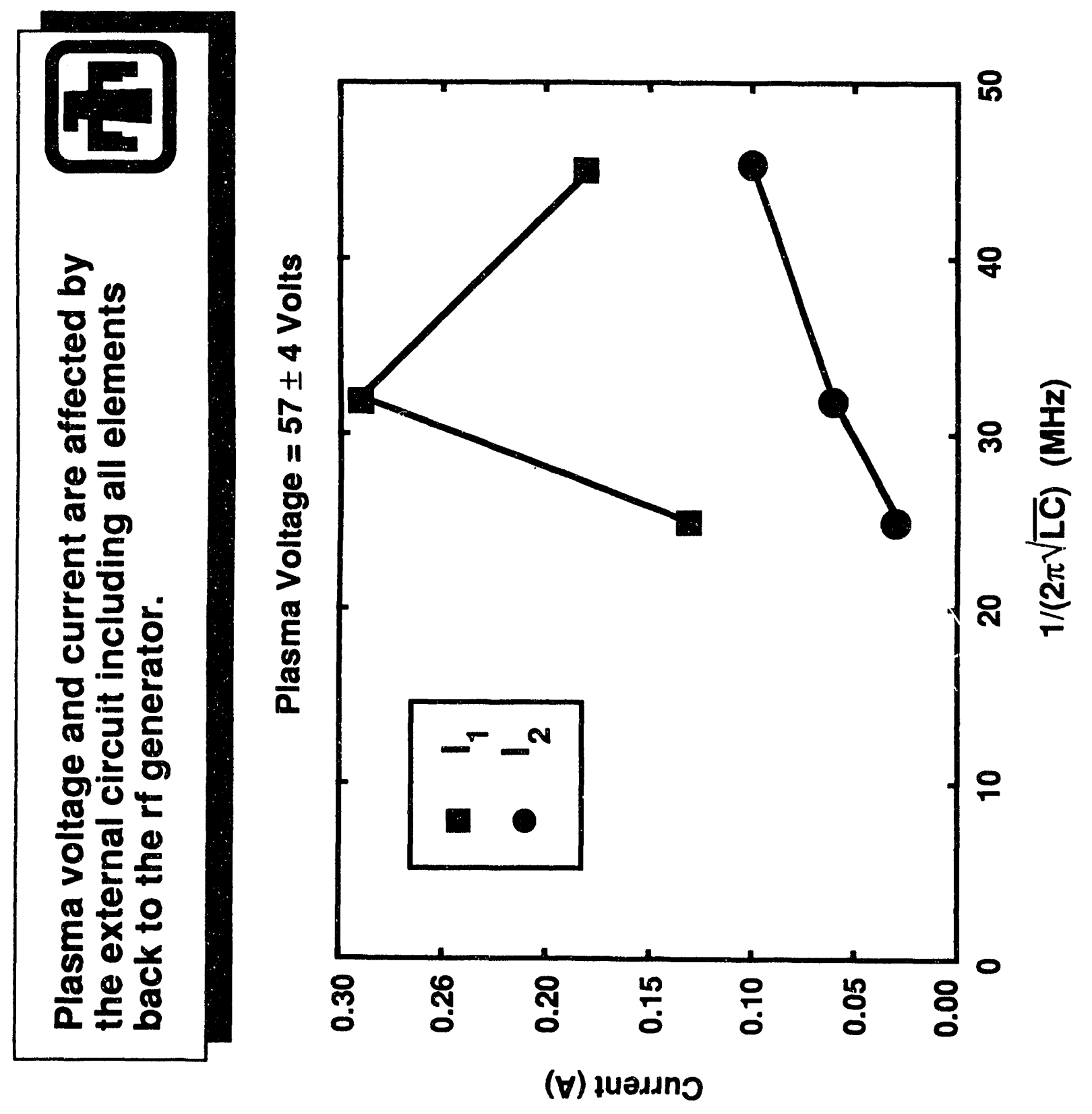




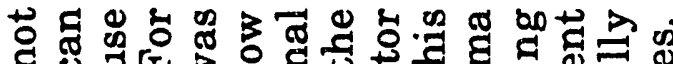

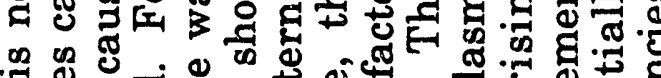
四

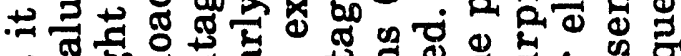

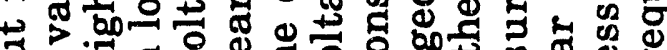

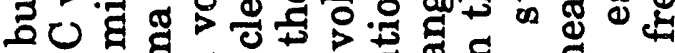

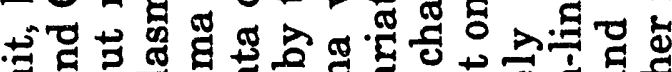
วิ สอم .

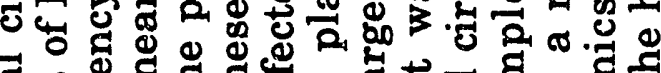
Ђె on ป

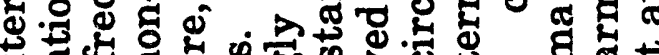

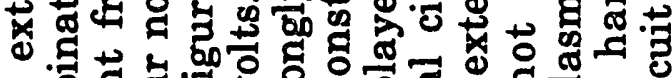

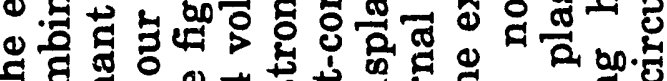

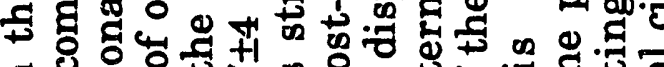

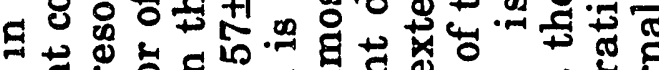
\& 연

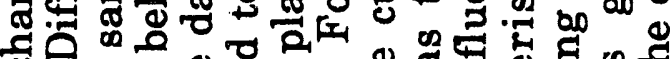

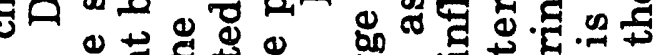
舟 \%

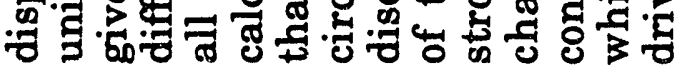

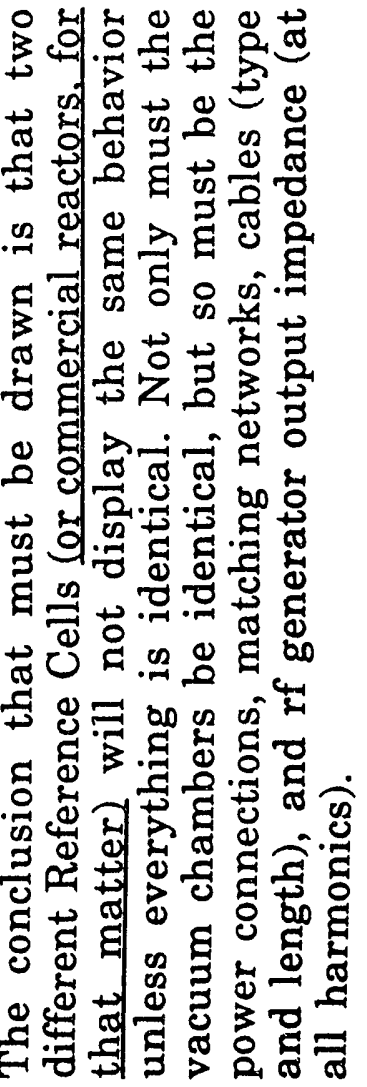

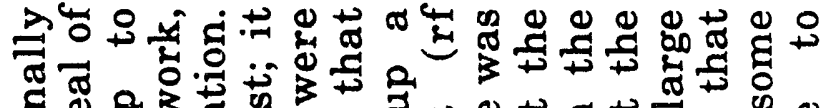

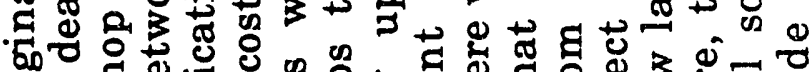

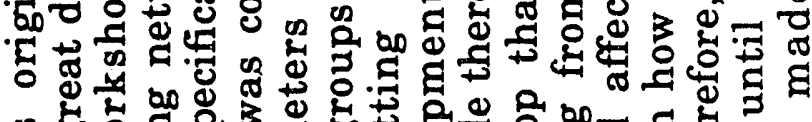
थ ๘

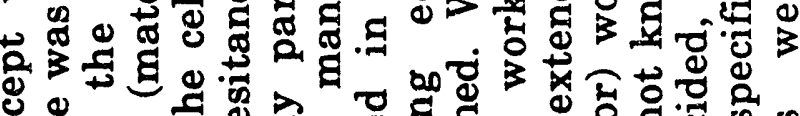

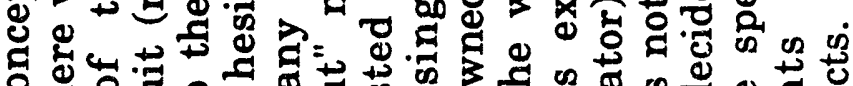

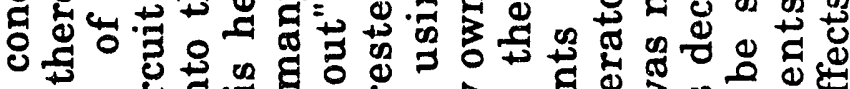

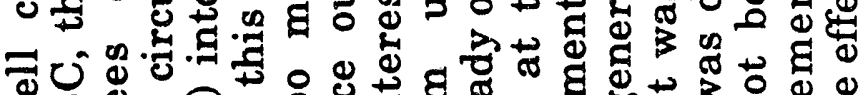

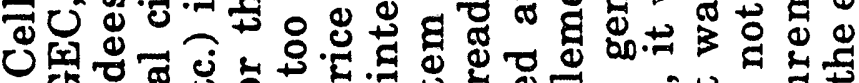

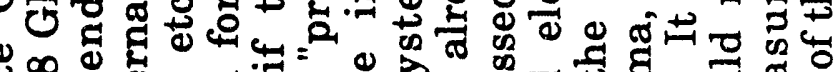

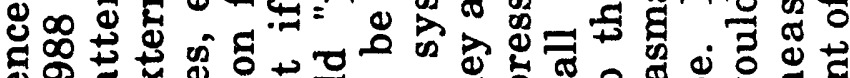

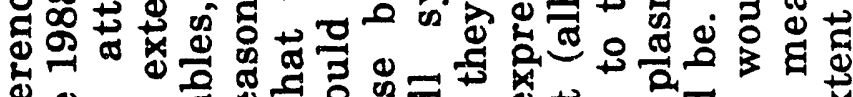

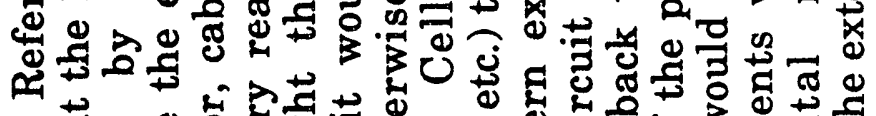

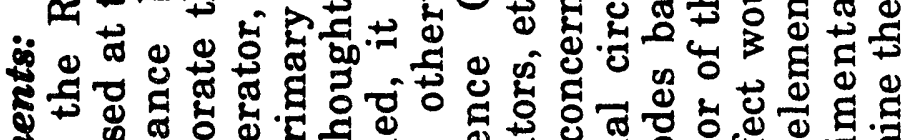
\%

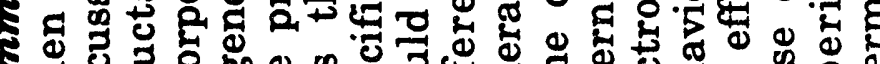
हญ อ

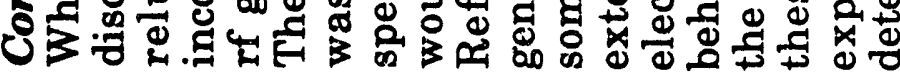

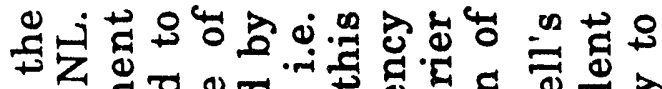

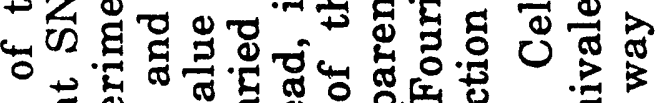

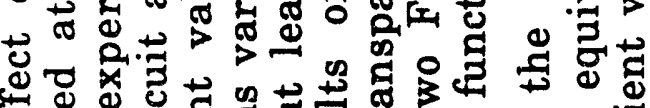

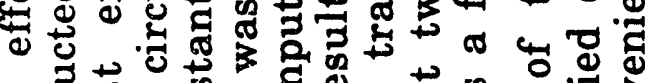
0 屯 \% 0.

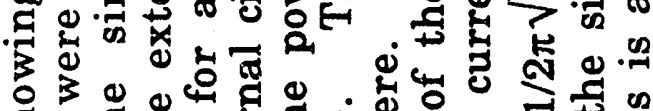

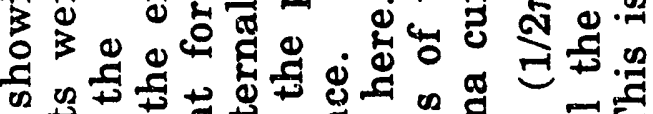

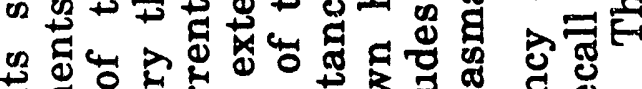
蛋

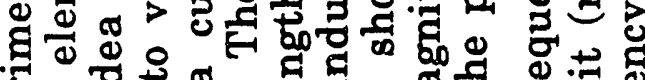
+ 汿 ช.

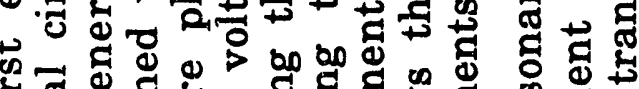
పే

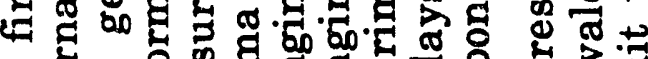

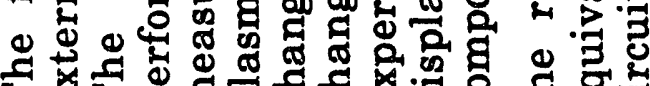

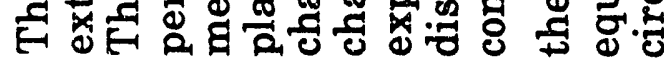



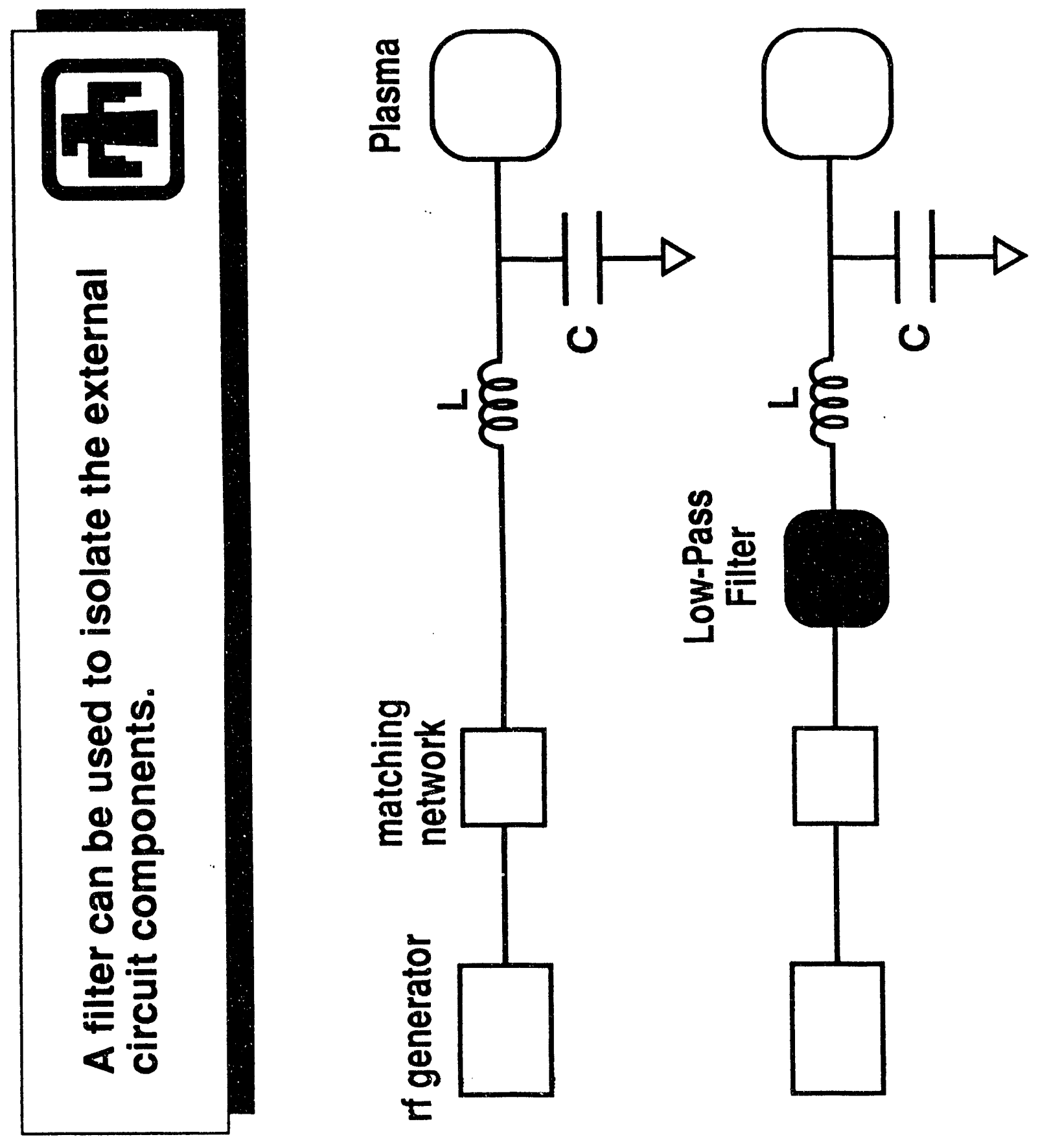


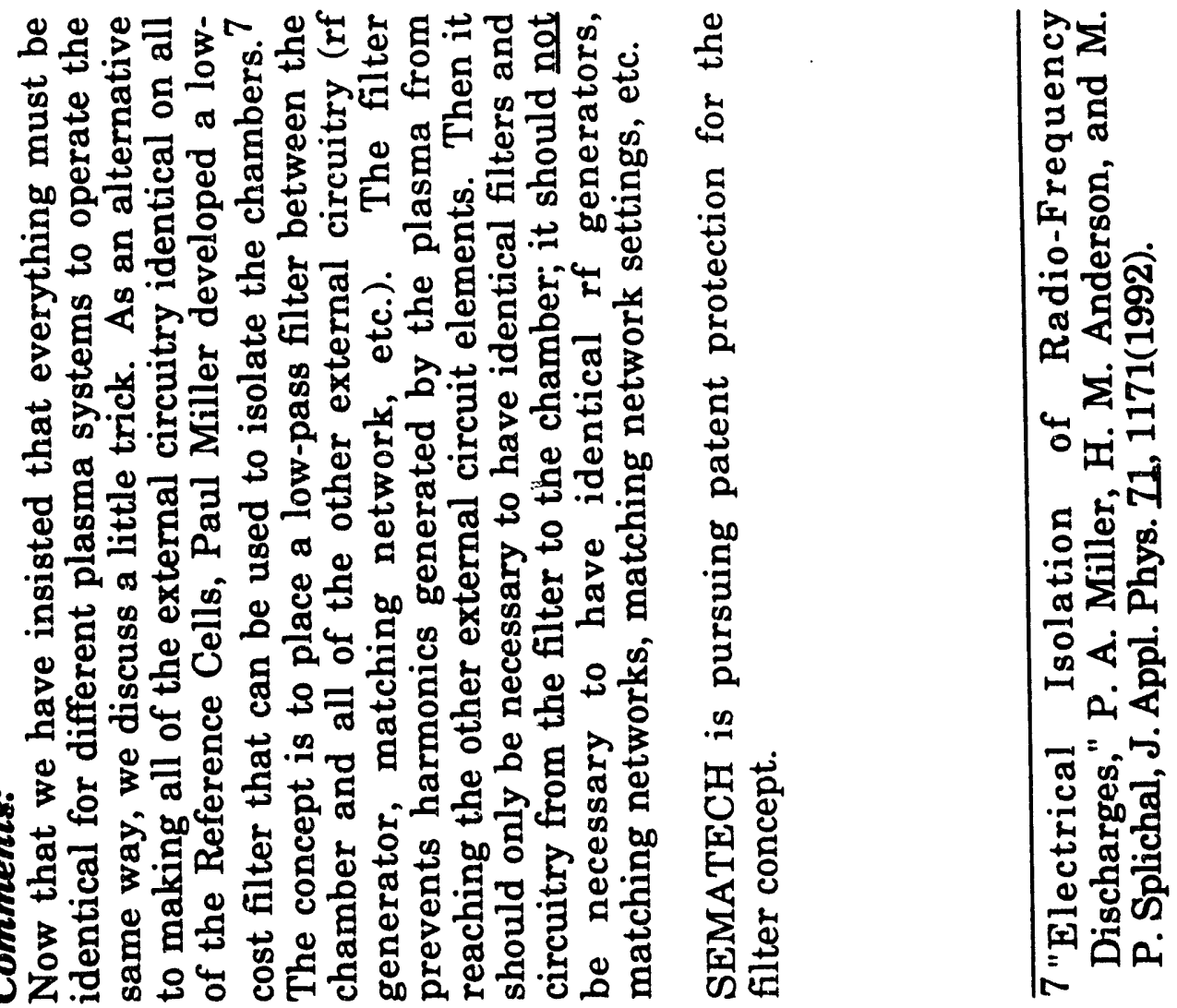



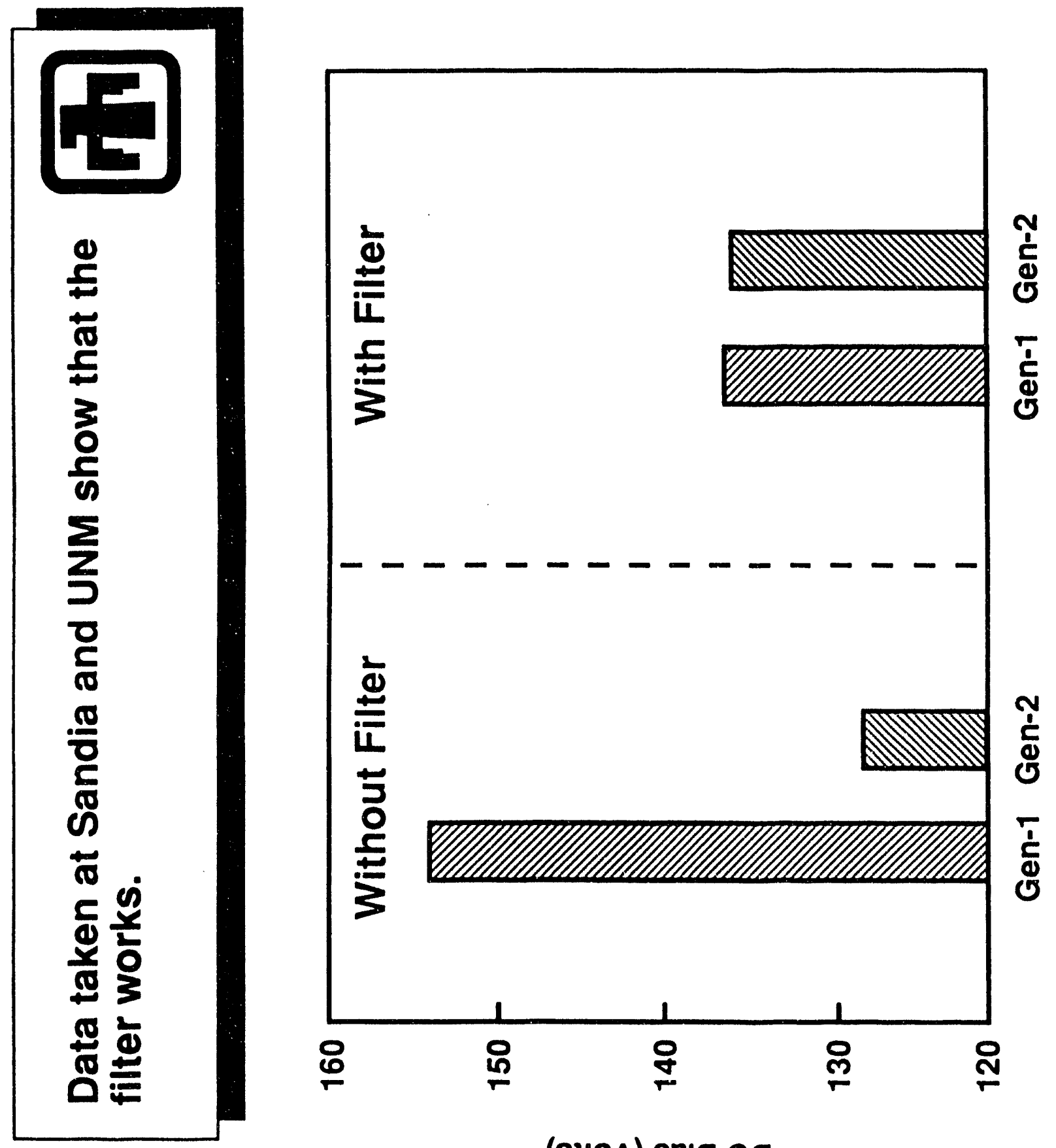

(shoN) SE!g Ja 


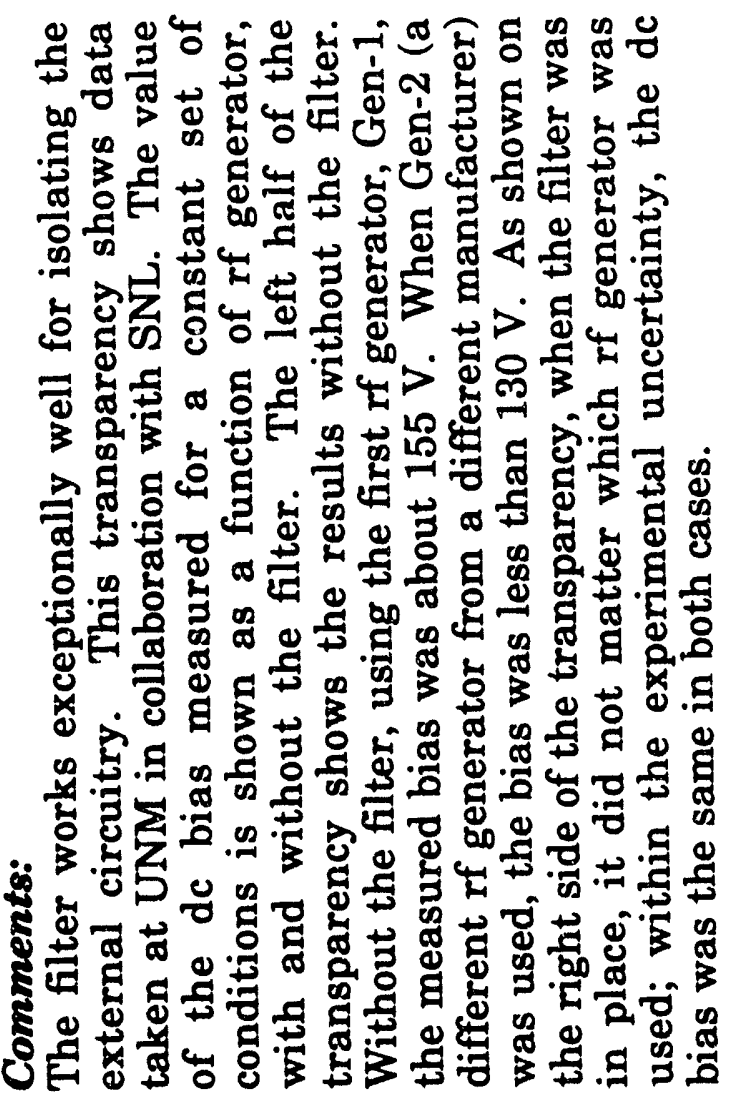




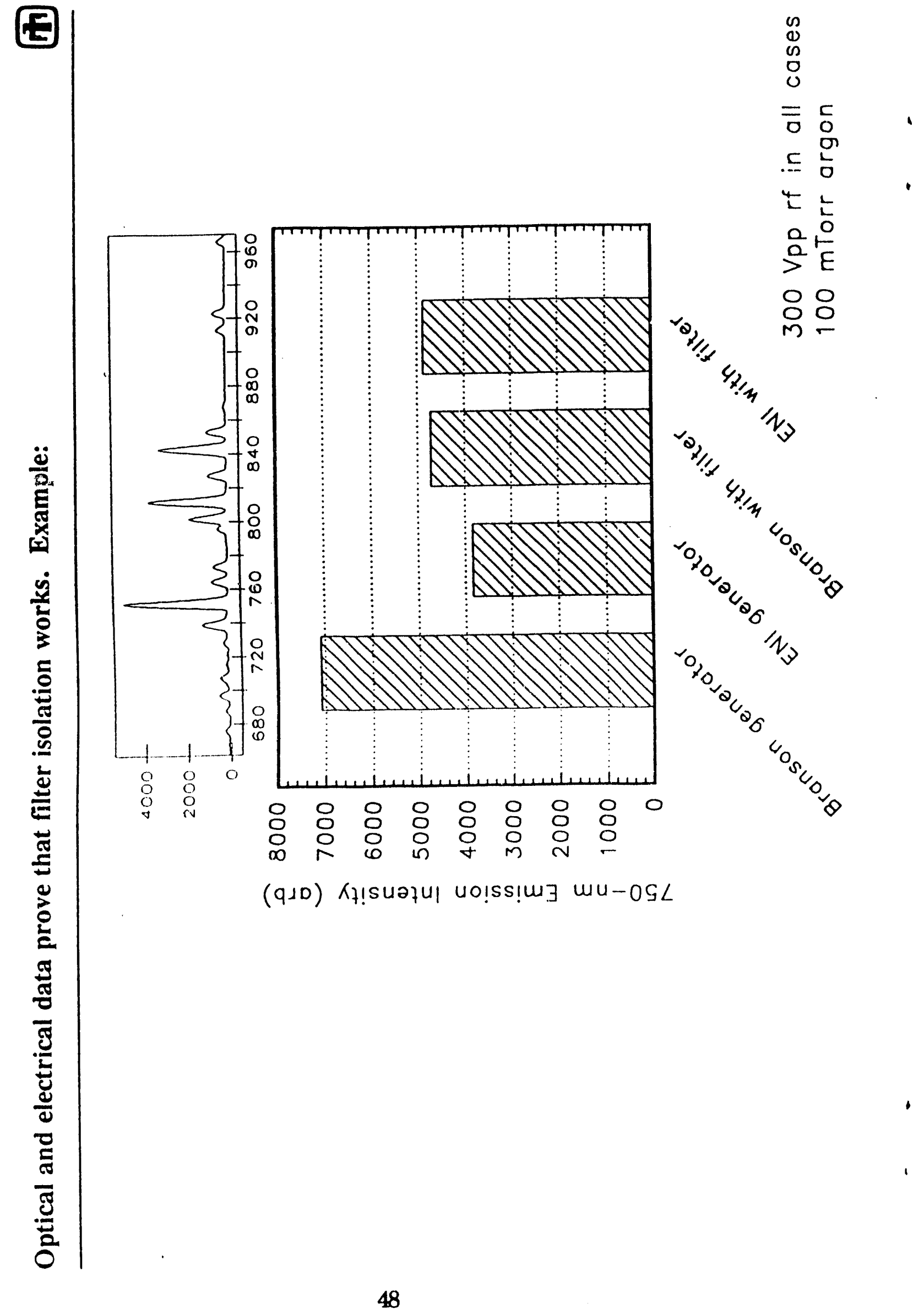




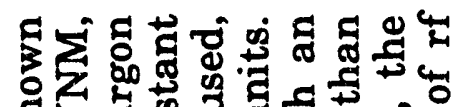

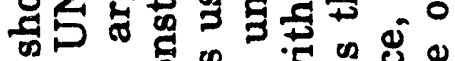
.

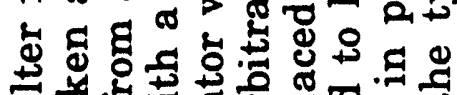

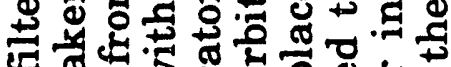
0 ్ㅕㅁ

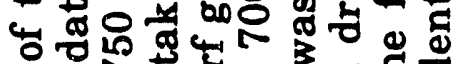
下先

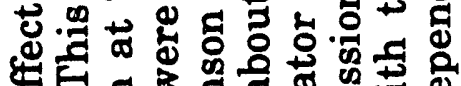

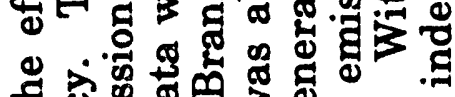

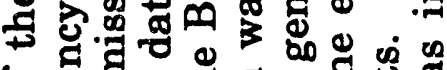

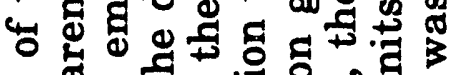

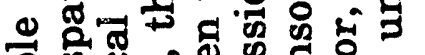

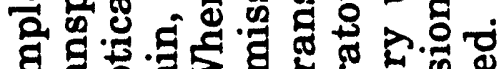
ต

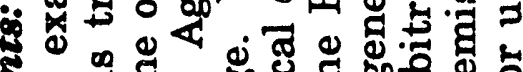

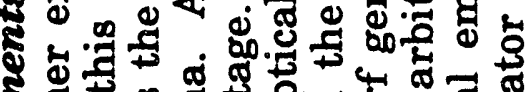

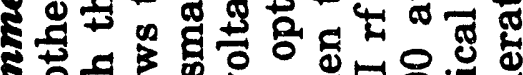

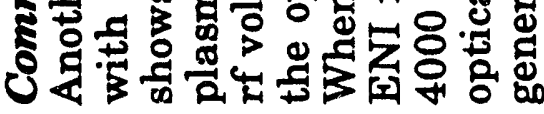

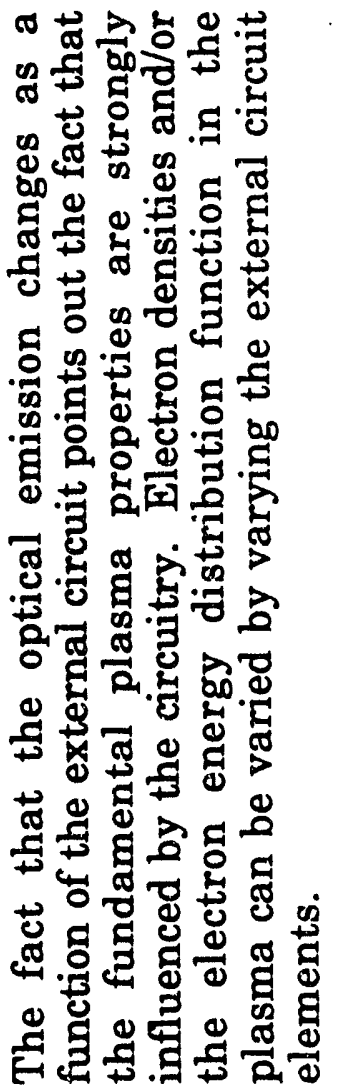

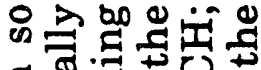

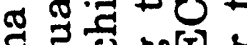

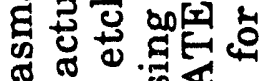
궁 009

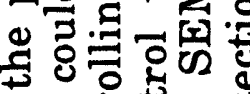
넉 节若 进 80 t

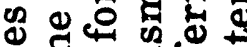
声 = $\frac{\pi}{0}$

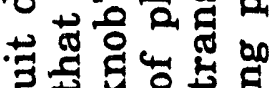

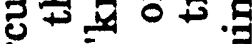
สี

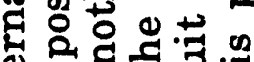
$\Phi$ ส

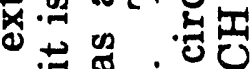
\&

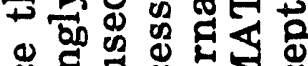
의 7 웡

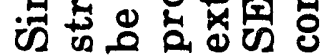




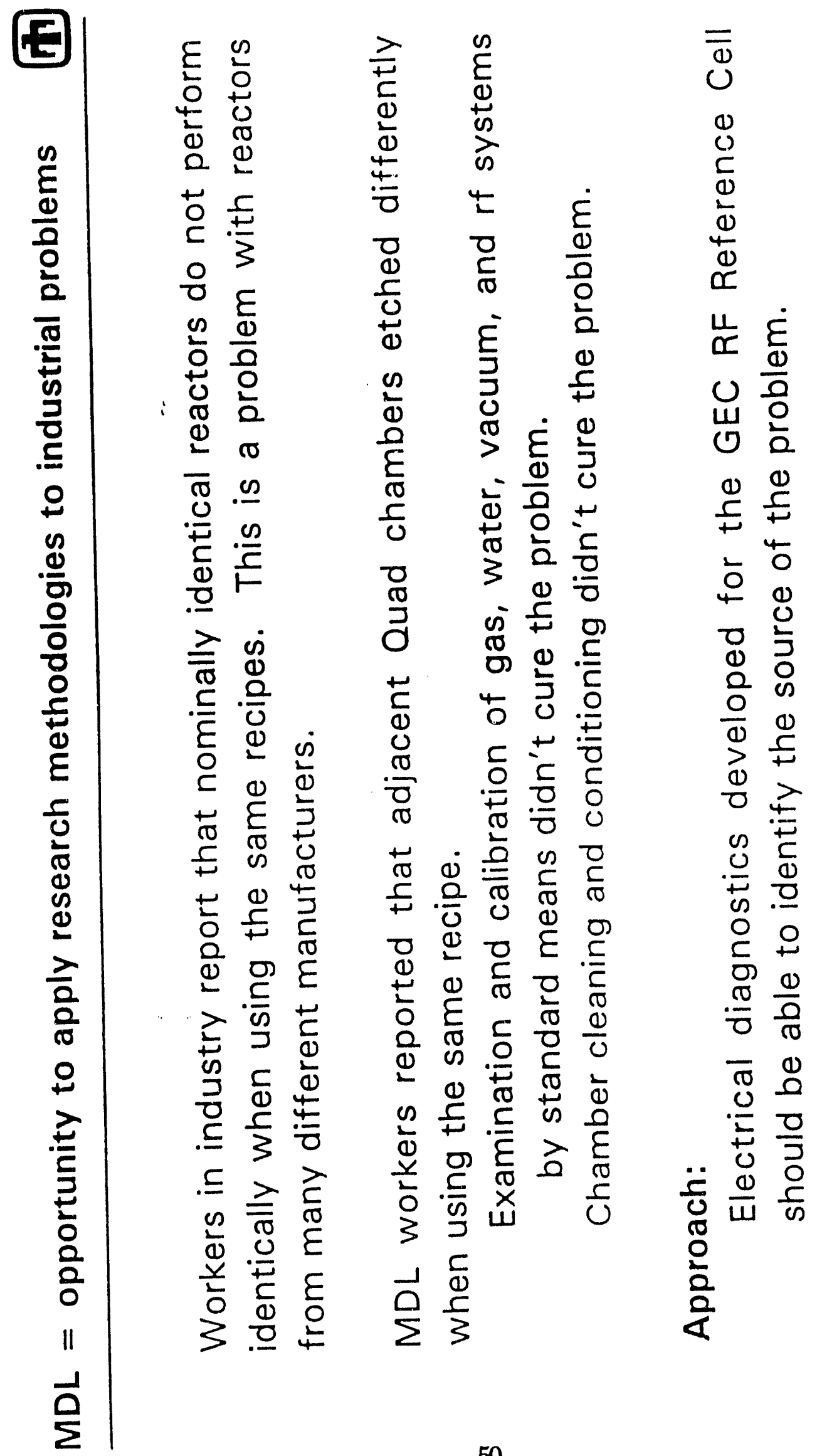




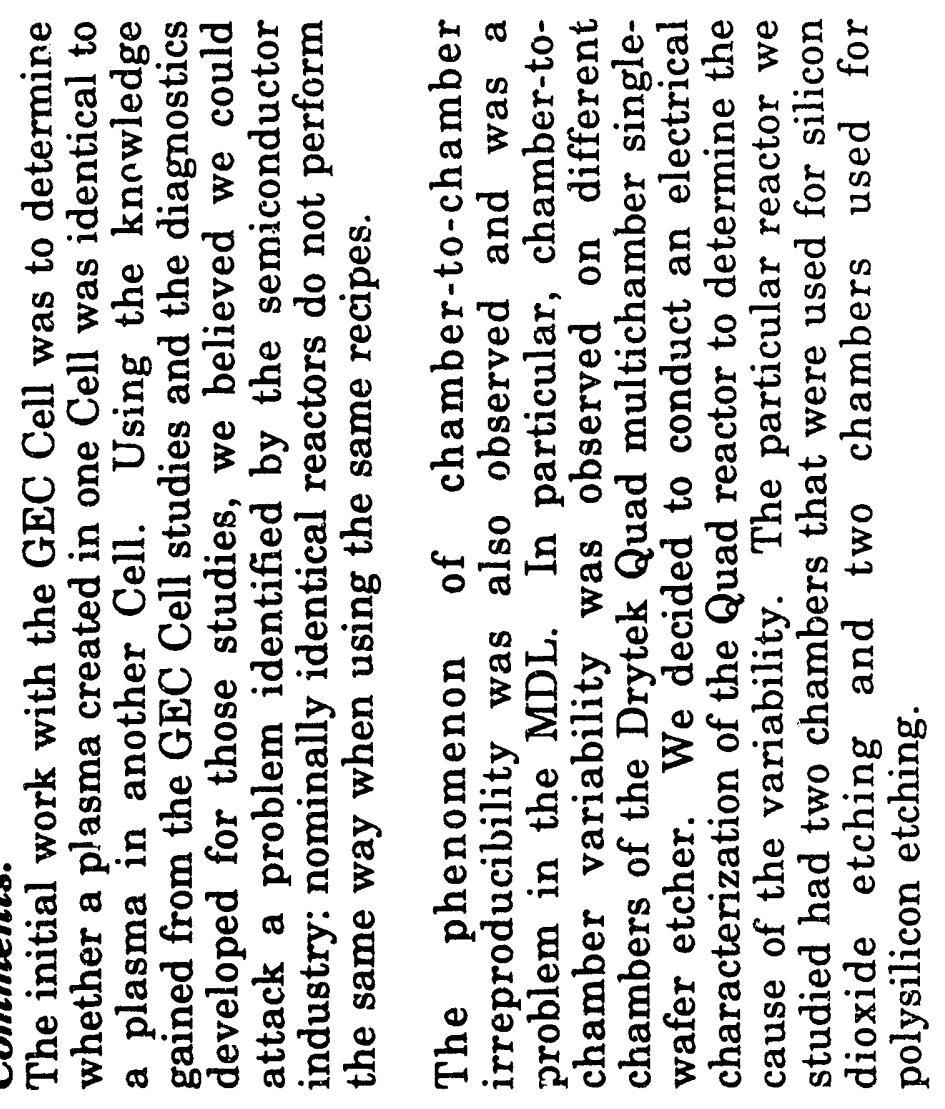




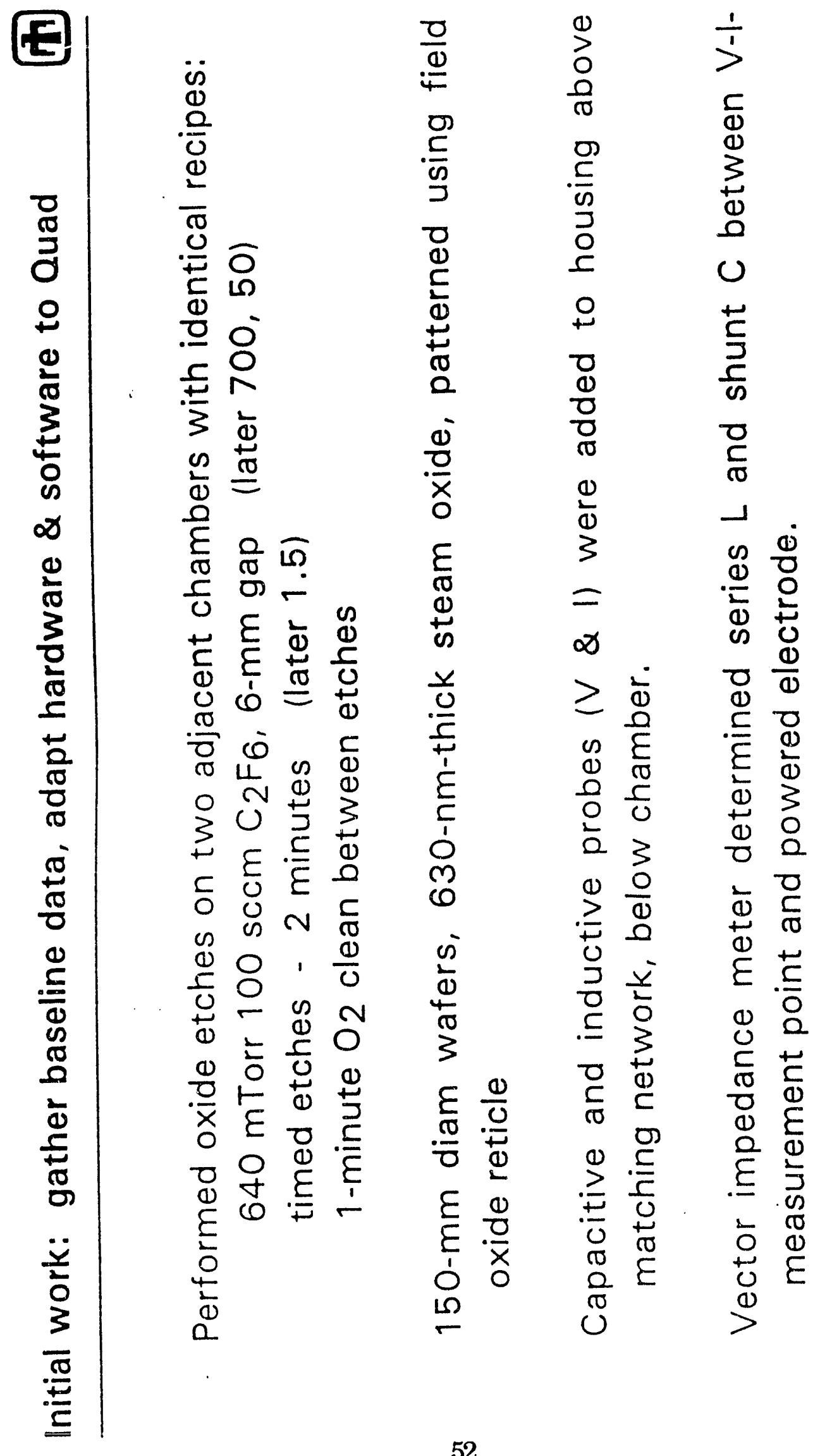




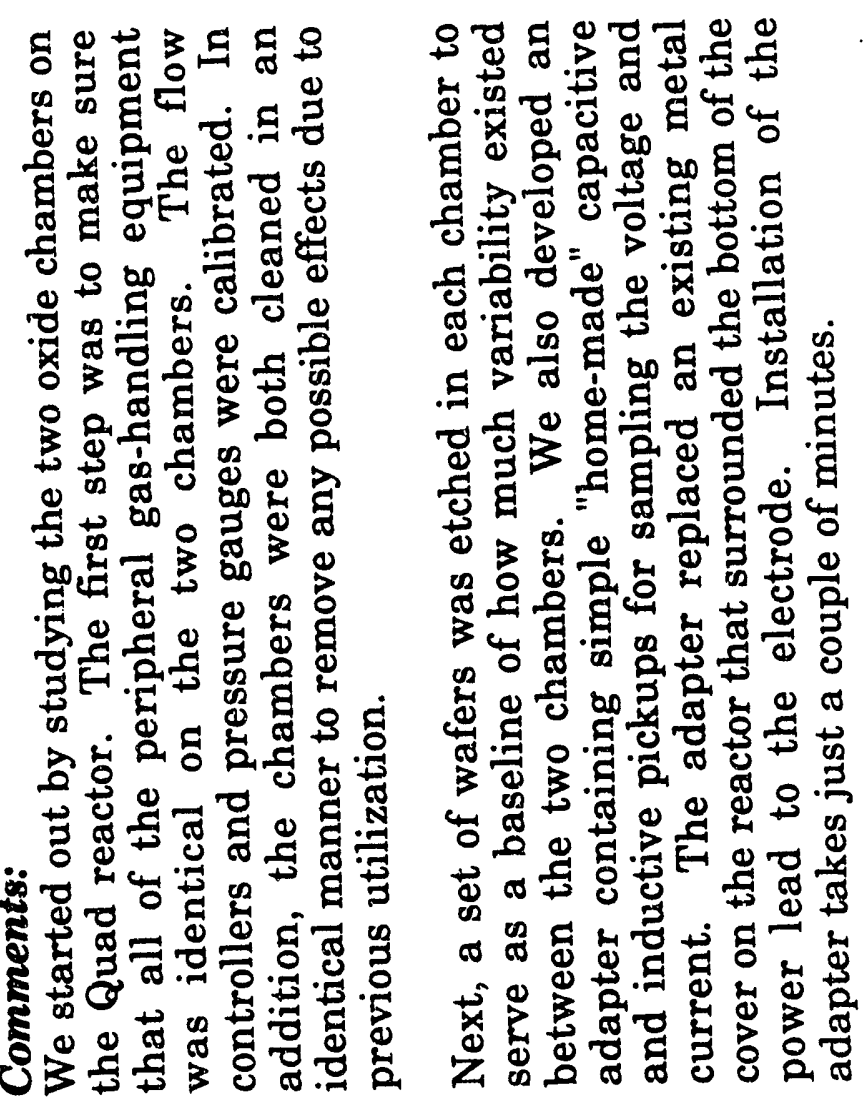




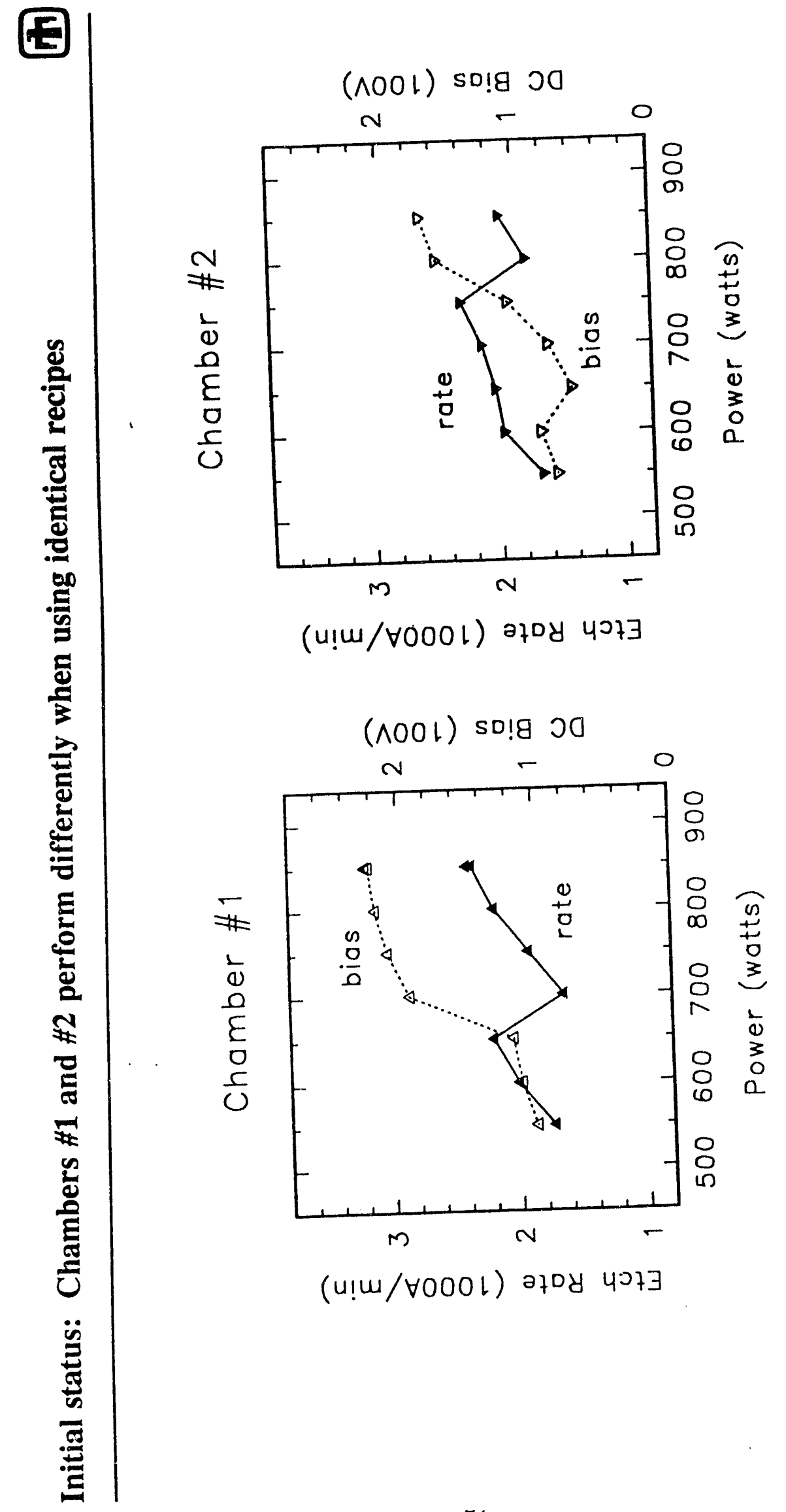



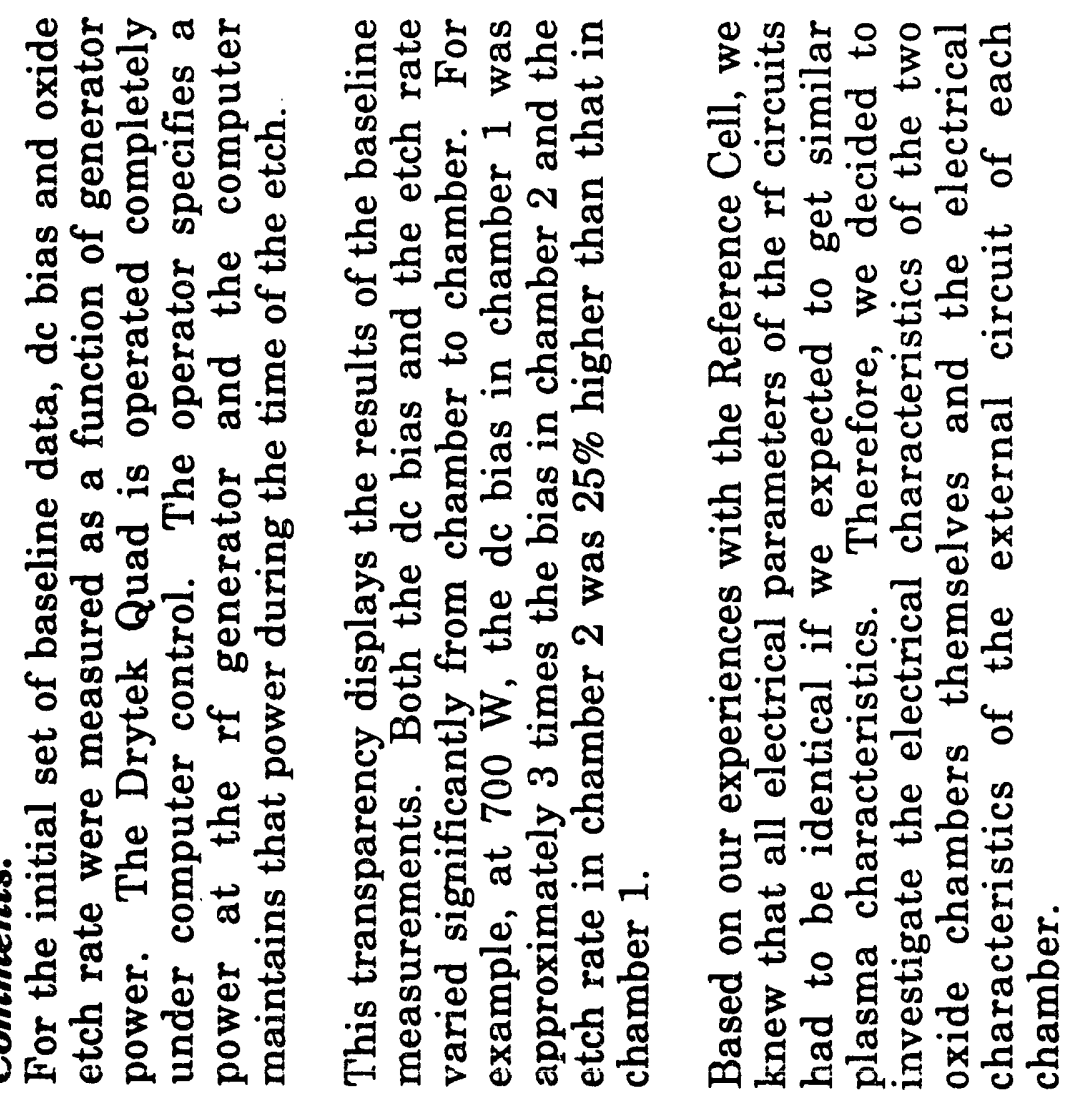

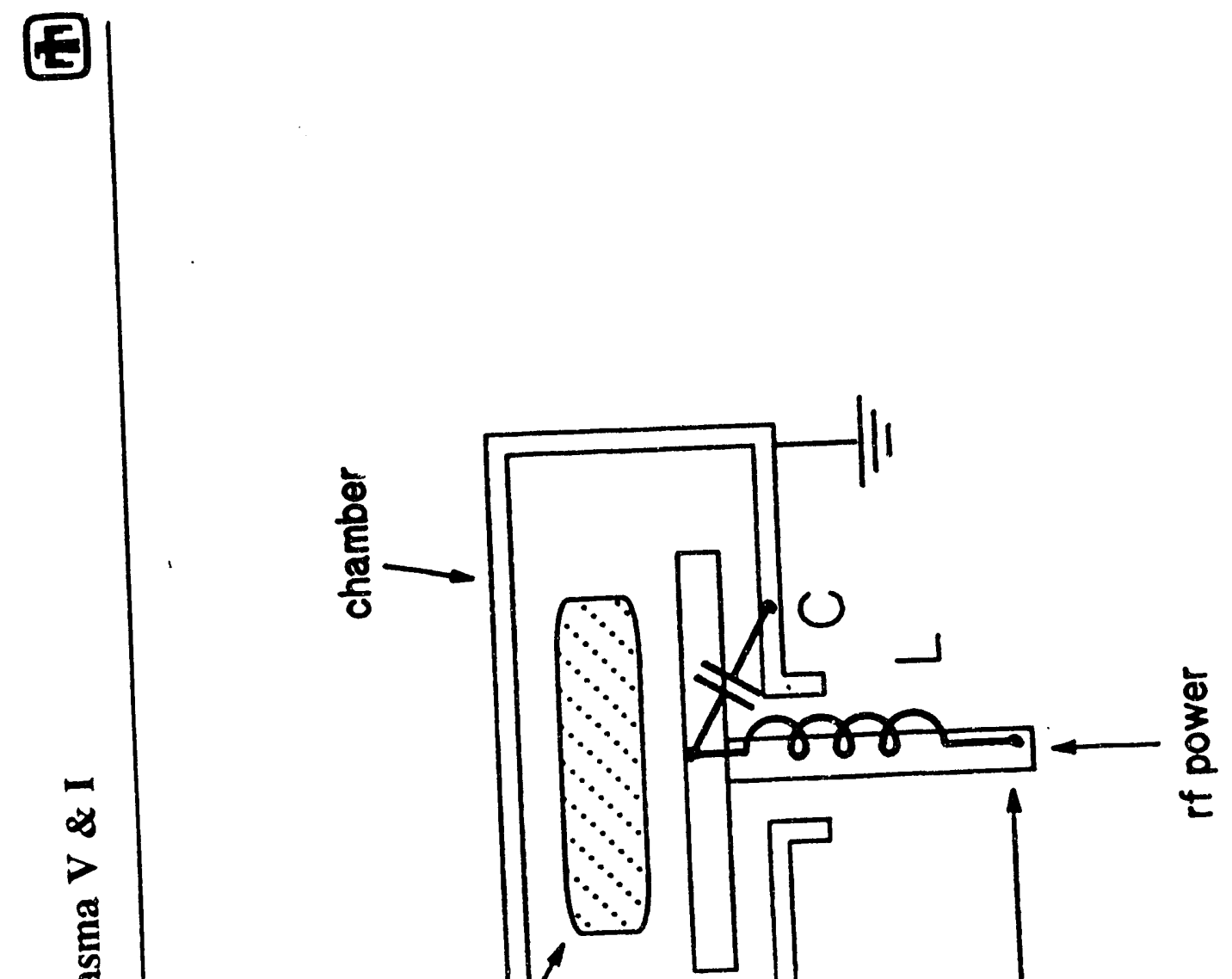

$\frac{2}{6}$

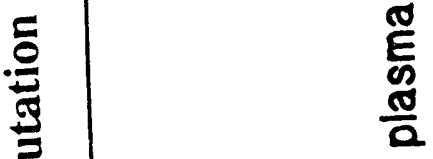

$\frac{\text { E }}{0}$

통

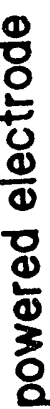

등
$\frac{7}{8}$
$\frac{0}{8}$
$\frac{8}{8}$
$\frac{0}{2}$
$\frac{0}{>}$ 


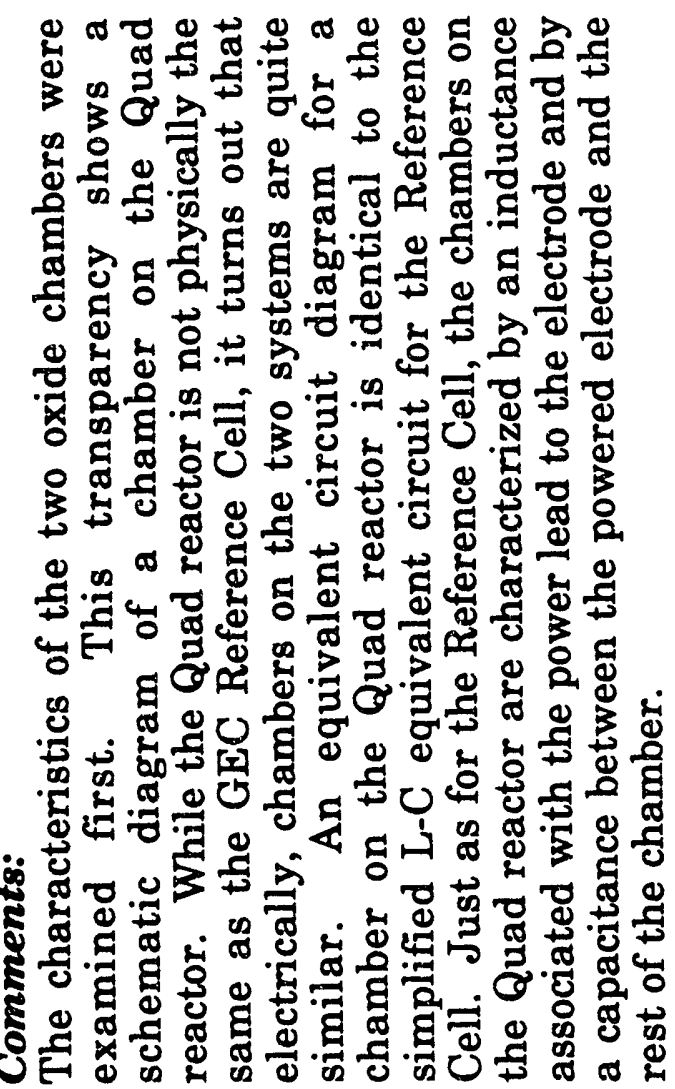




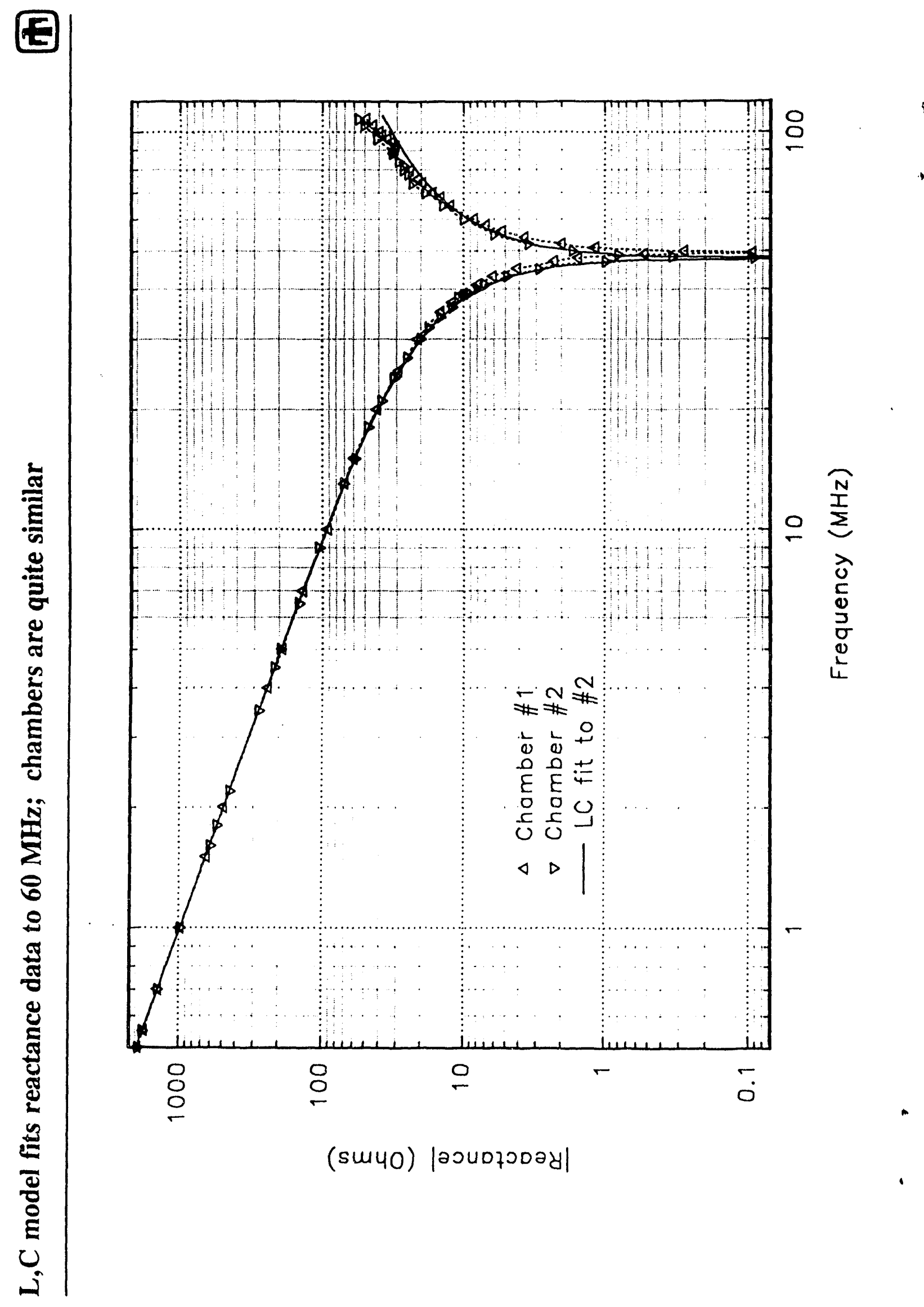




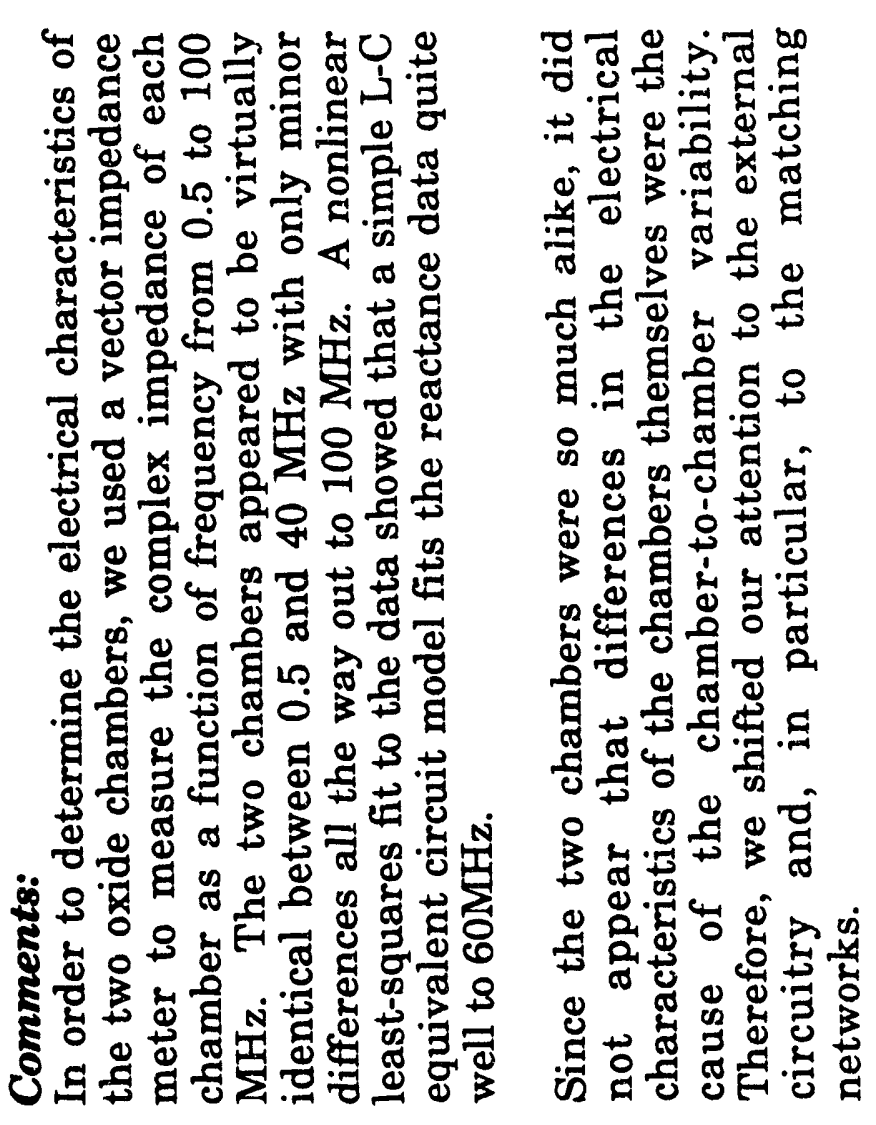




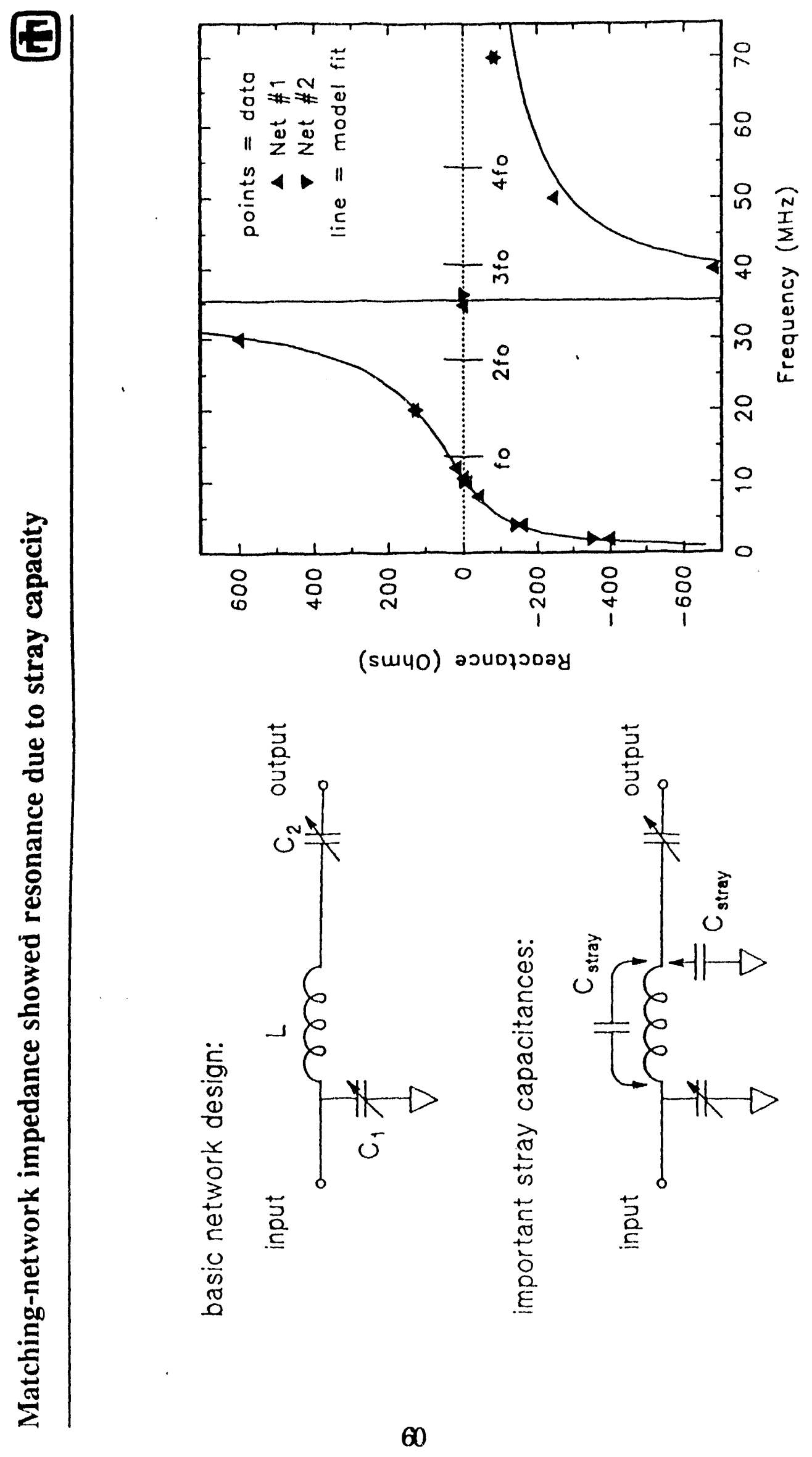




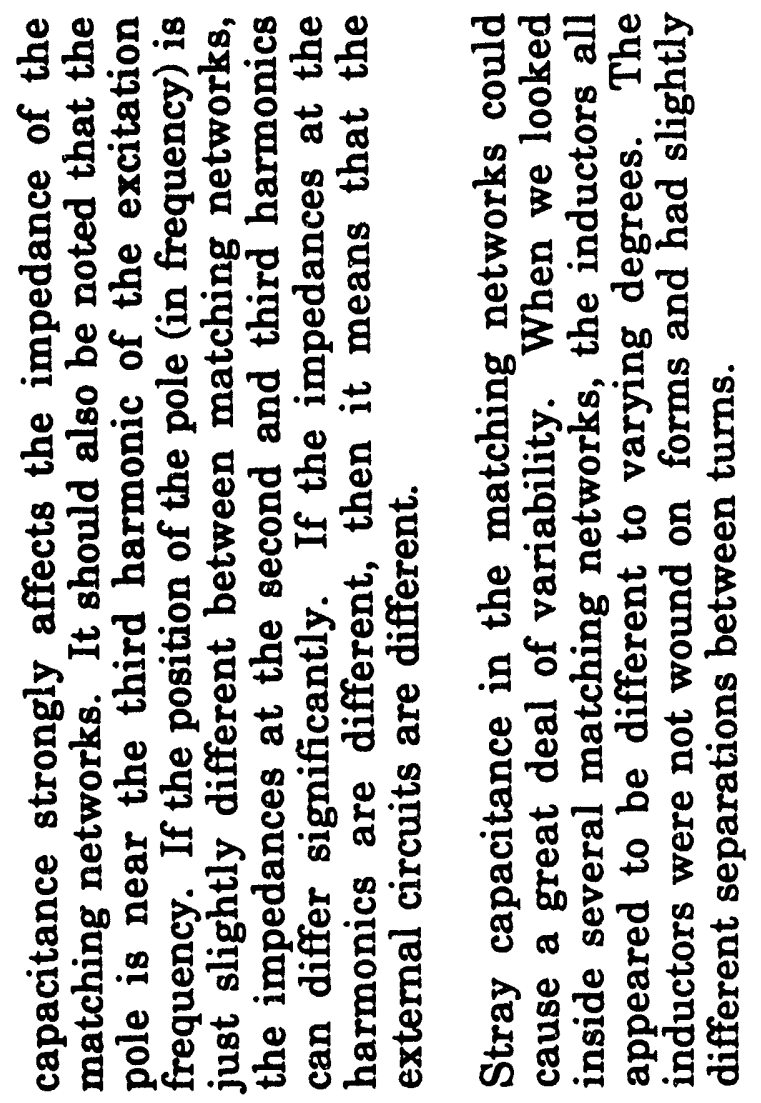

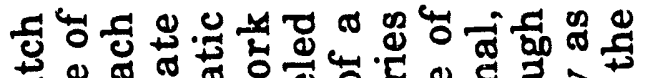

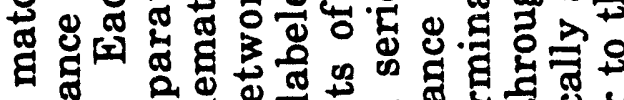
융. 语 里虽出 可.

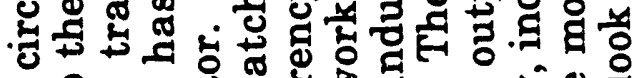

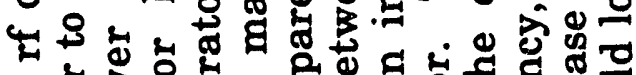

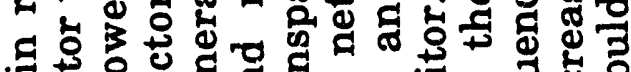
ช

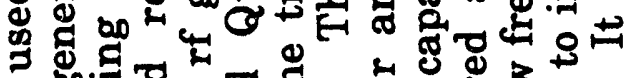

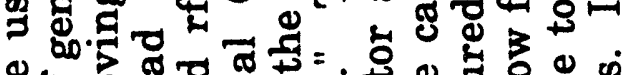

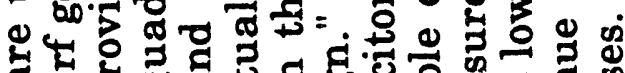
๘

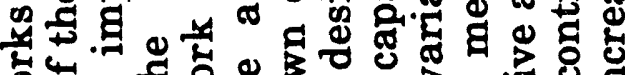

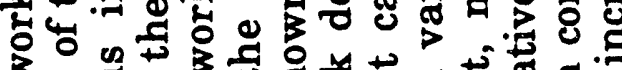
올

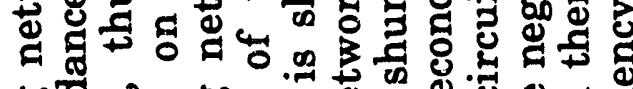

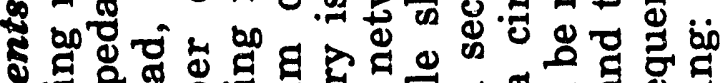

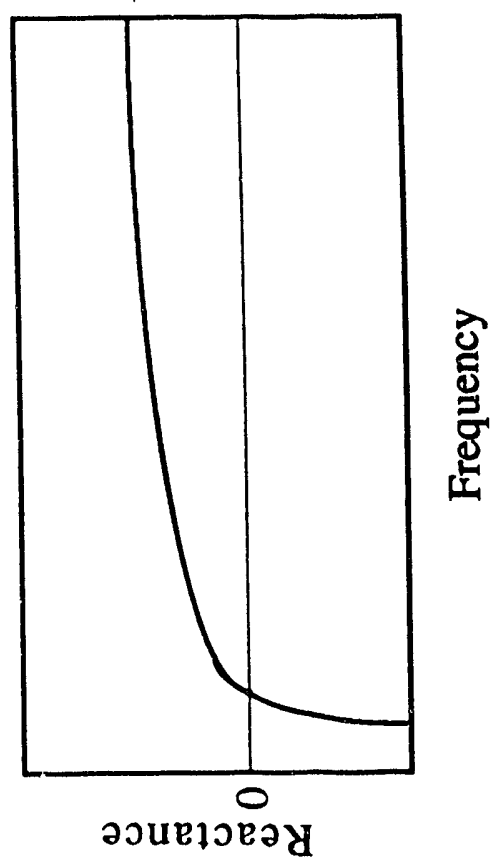

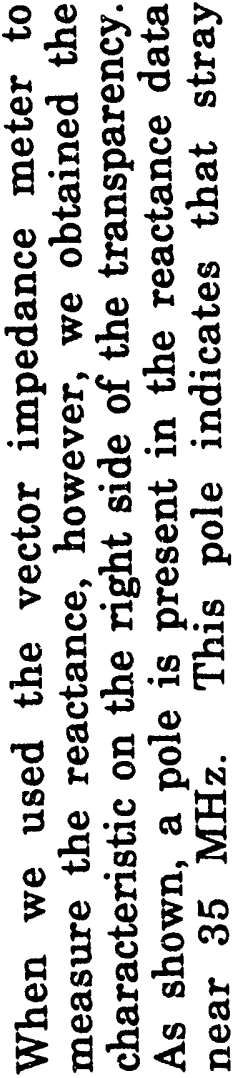




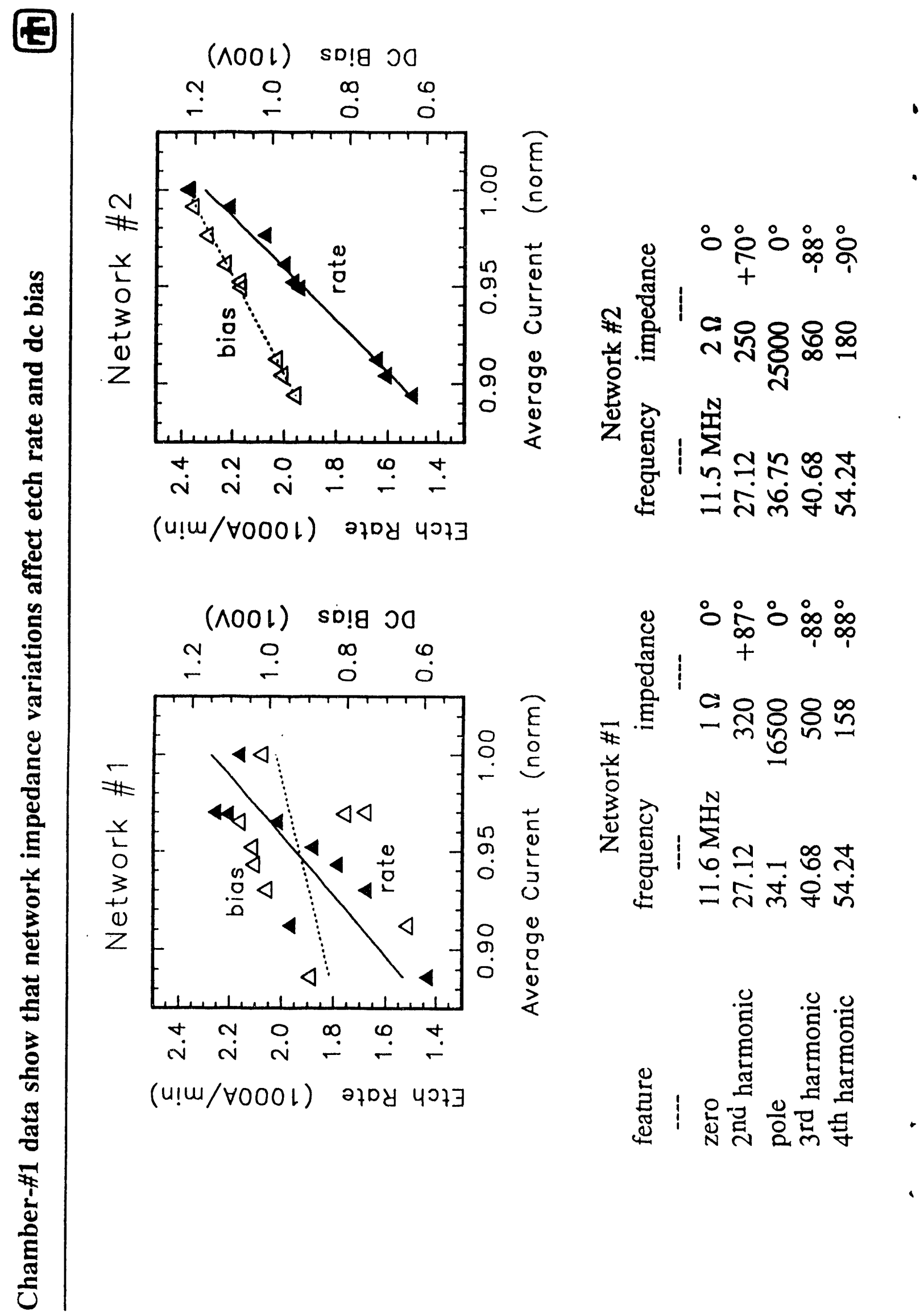




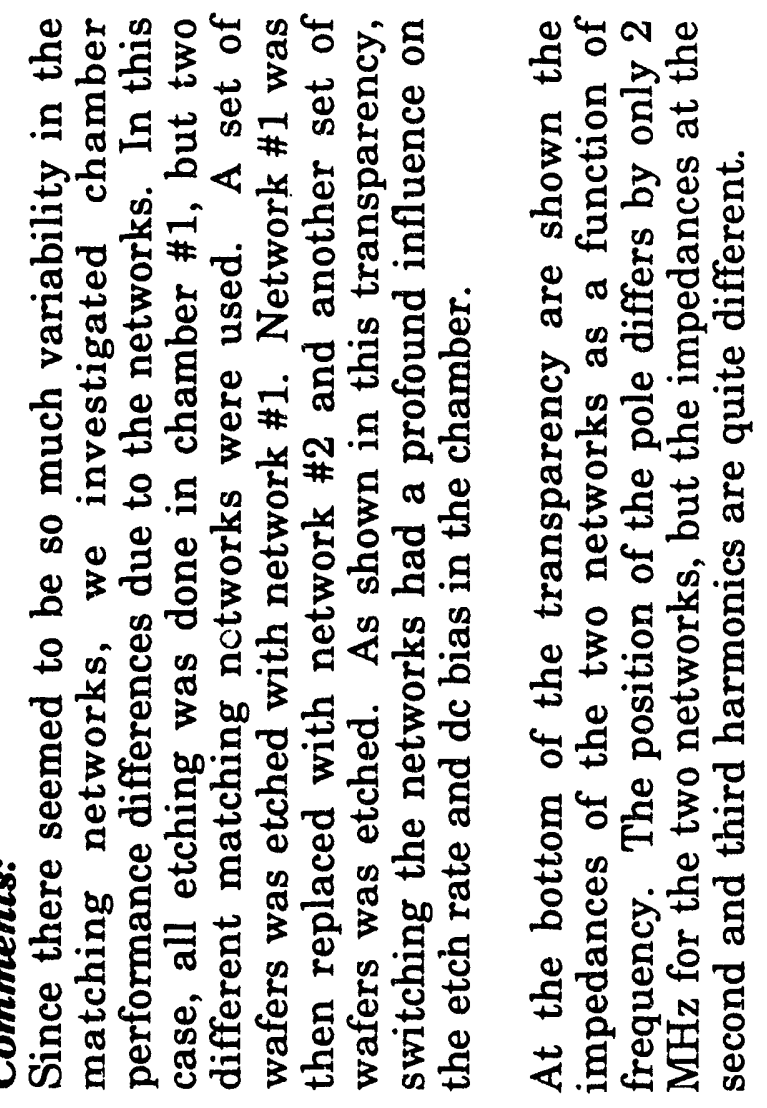




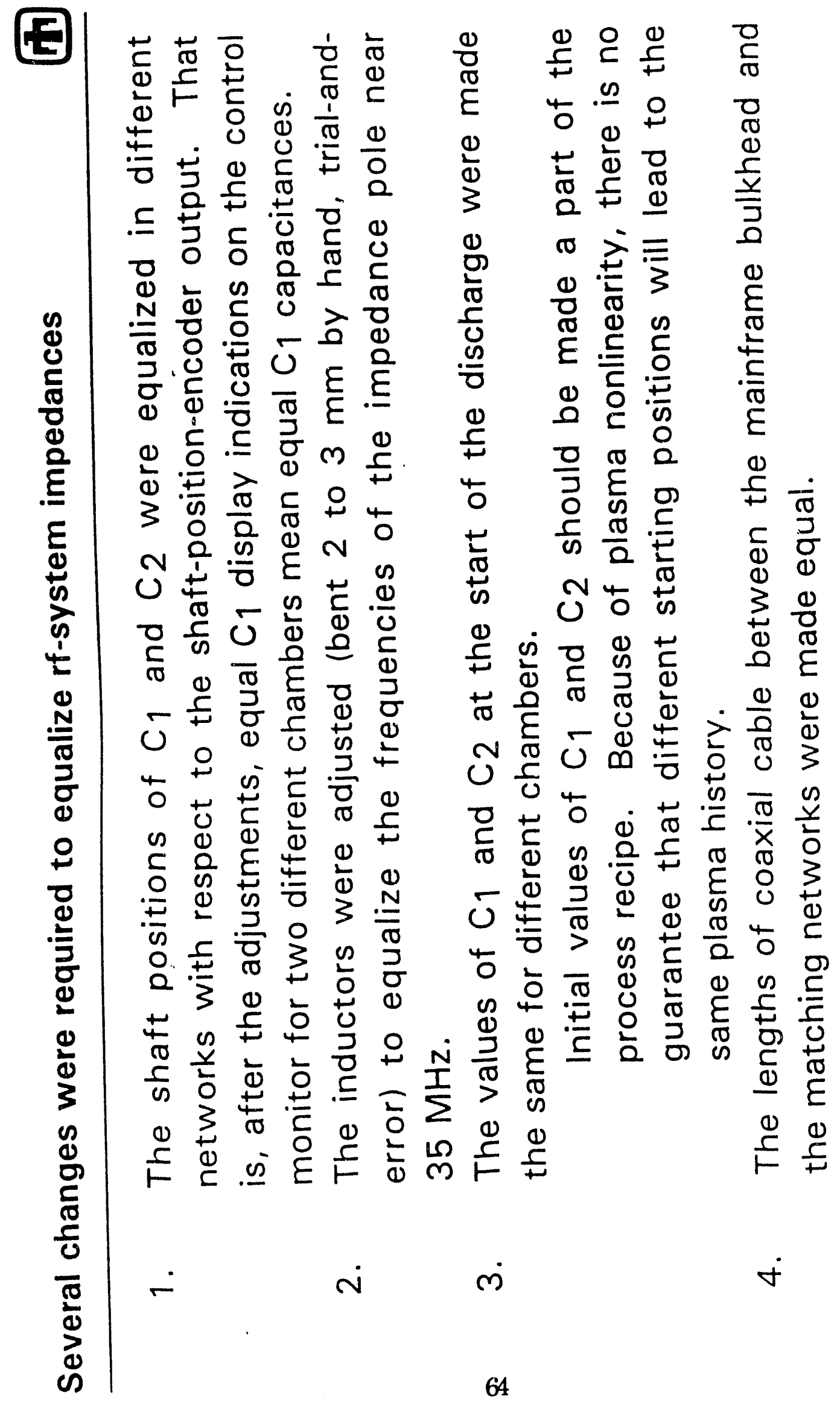




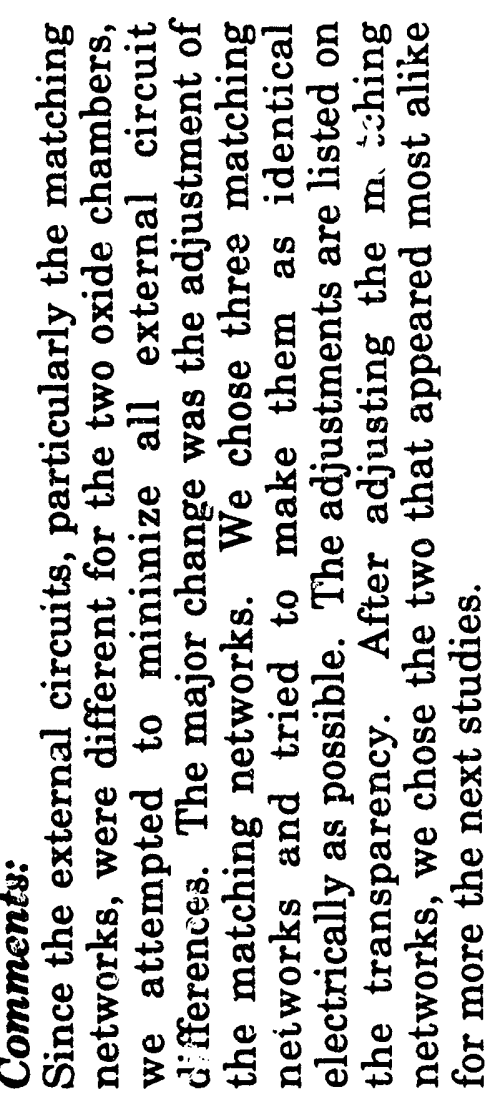




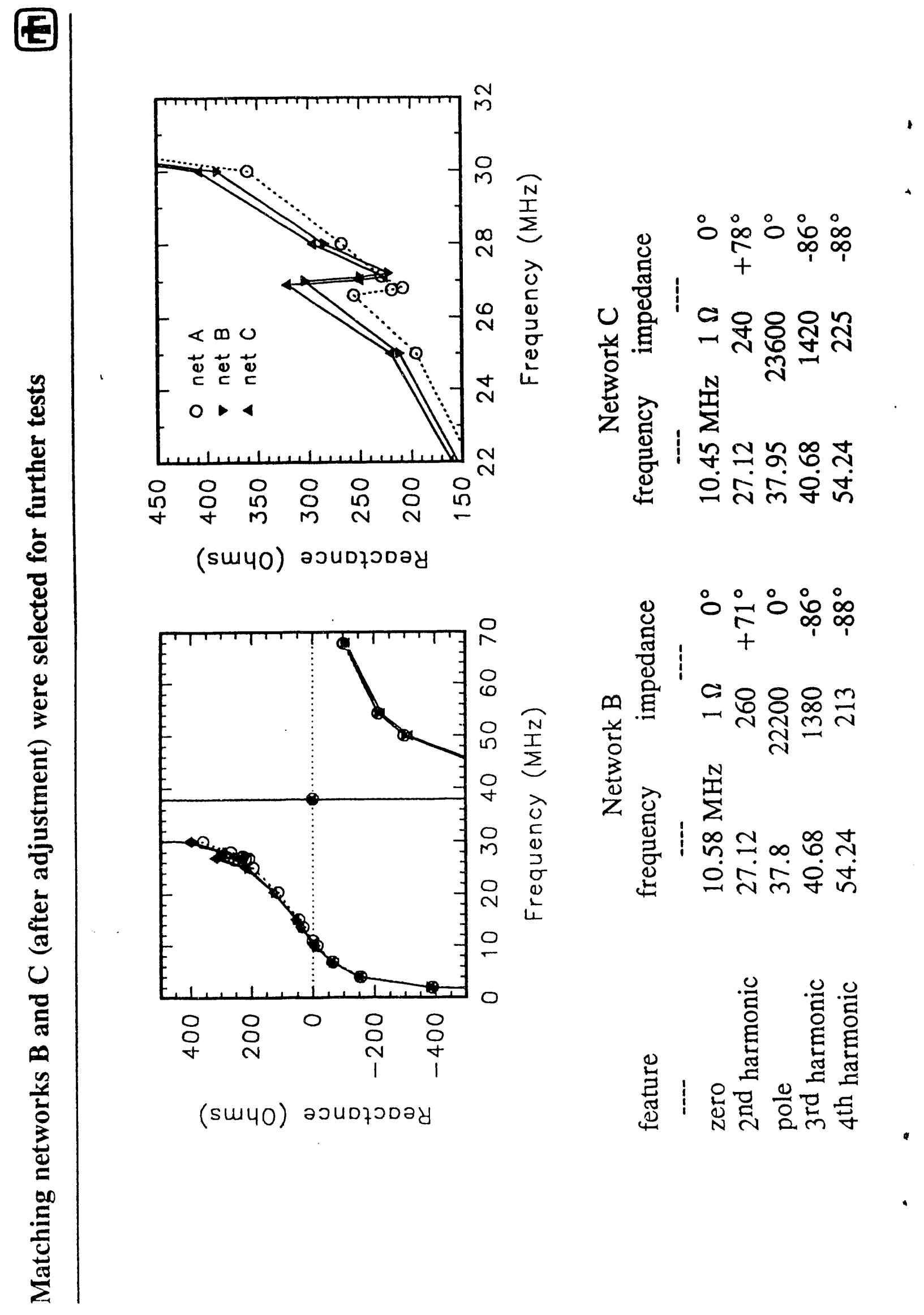




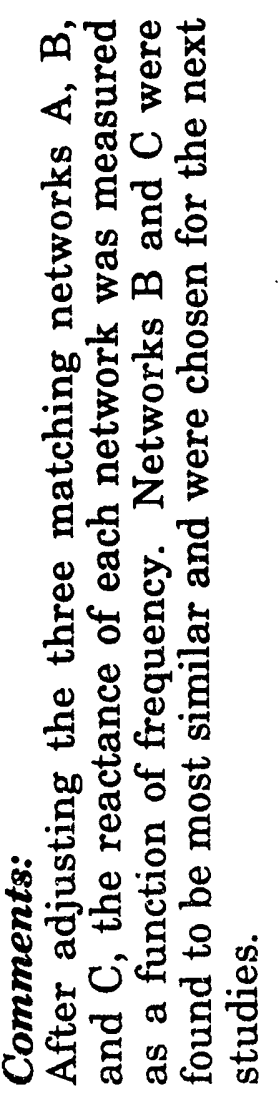




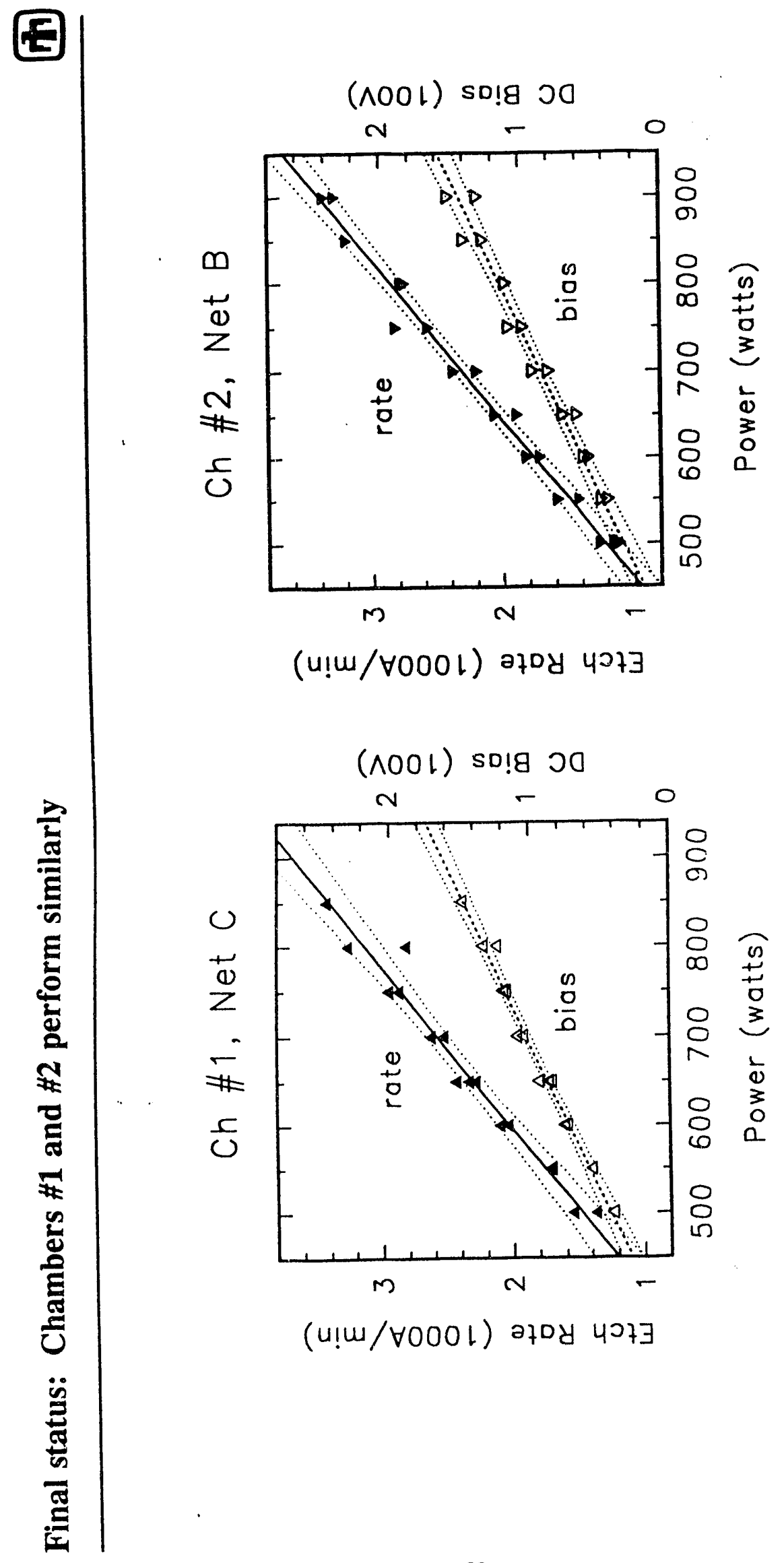




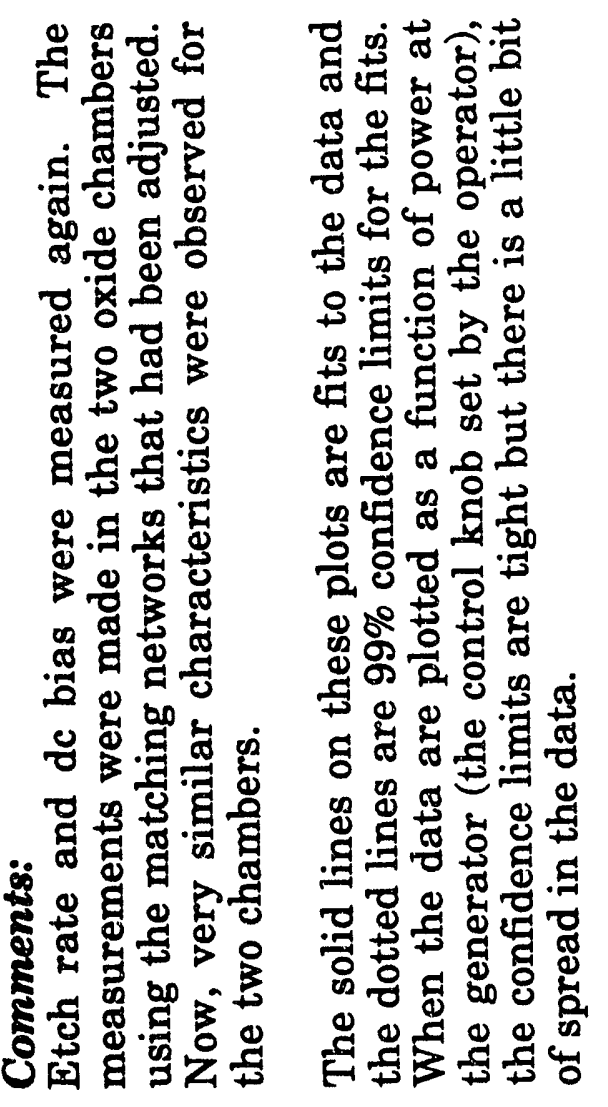




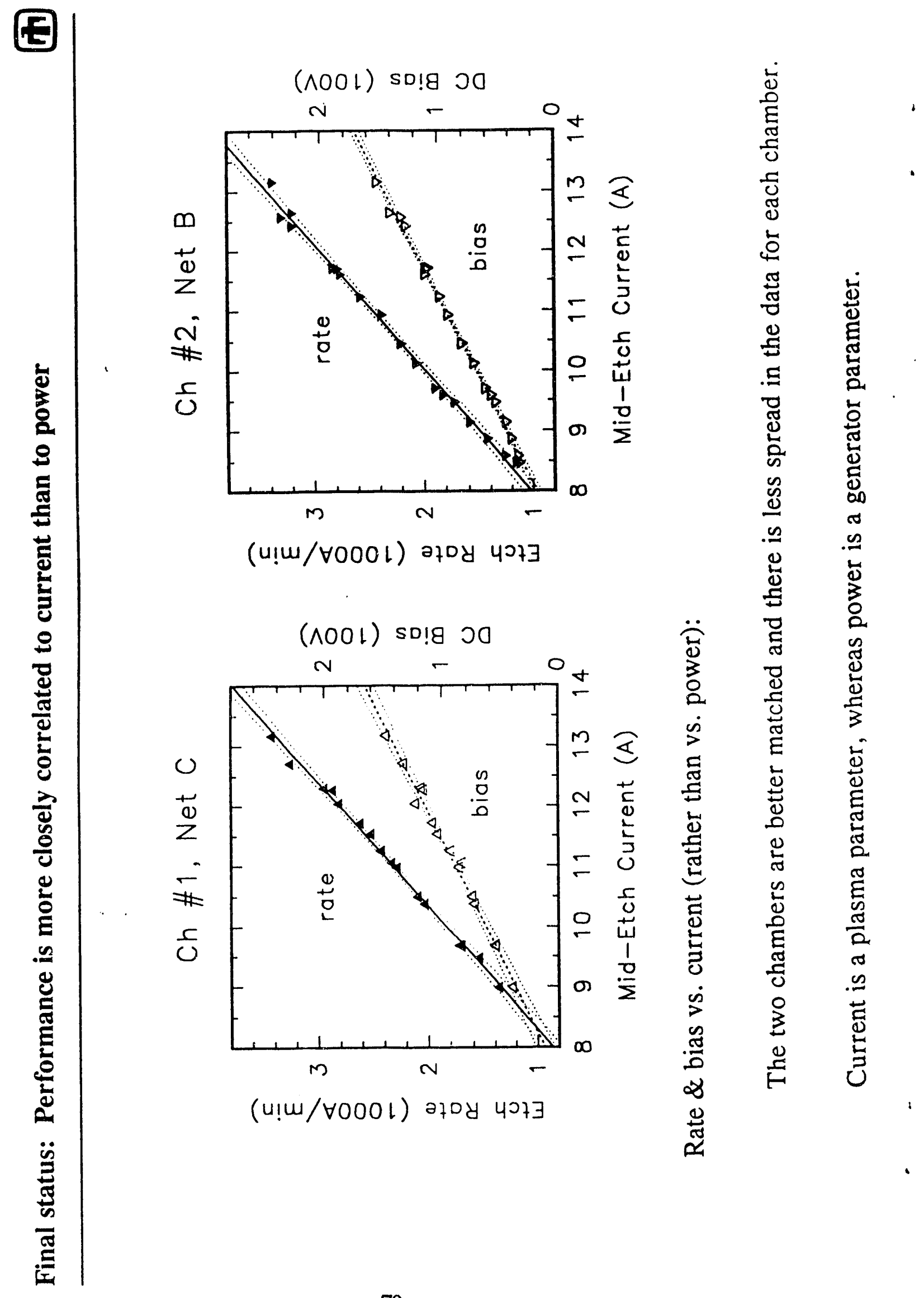




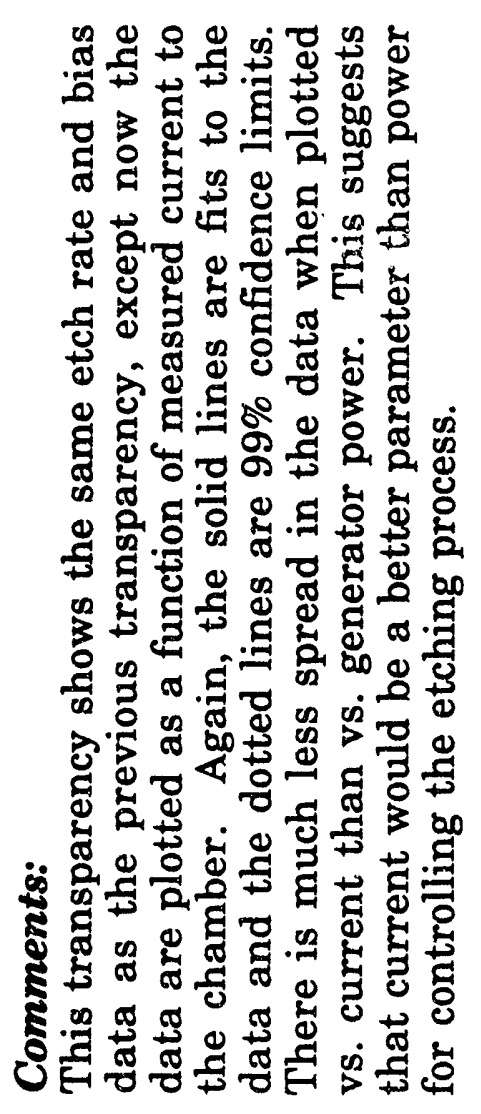




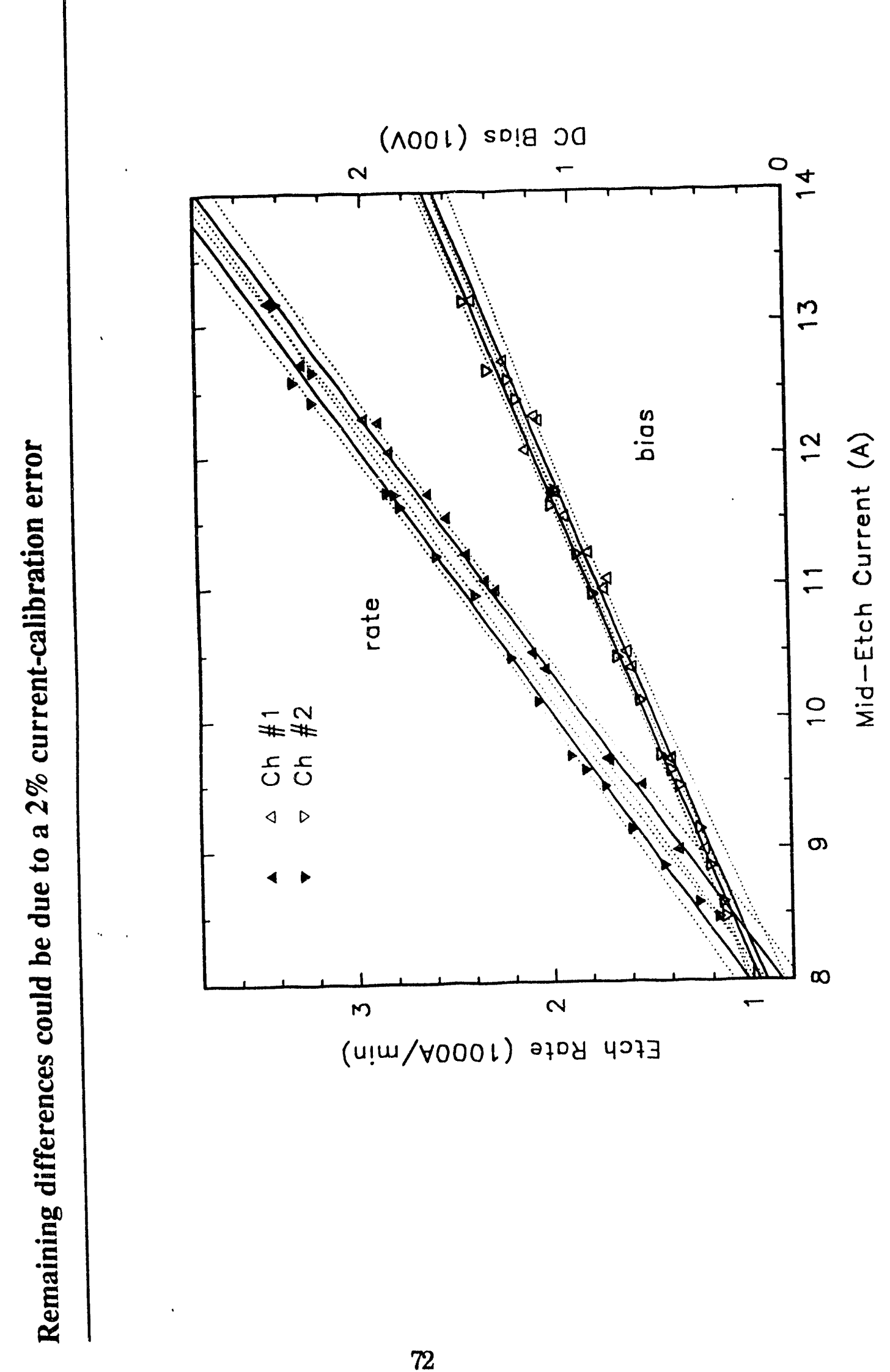




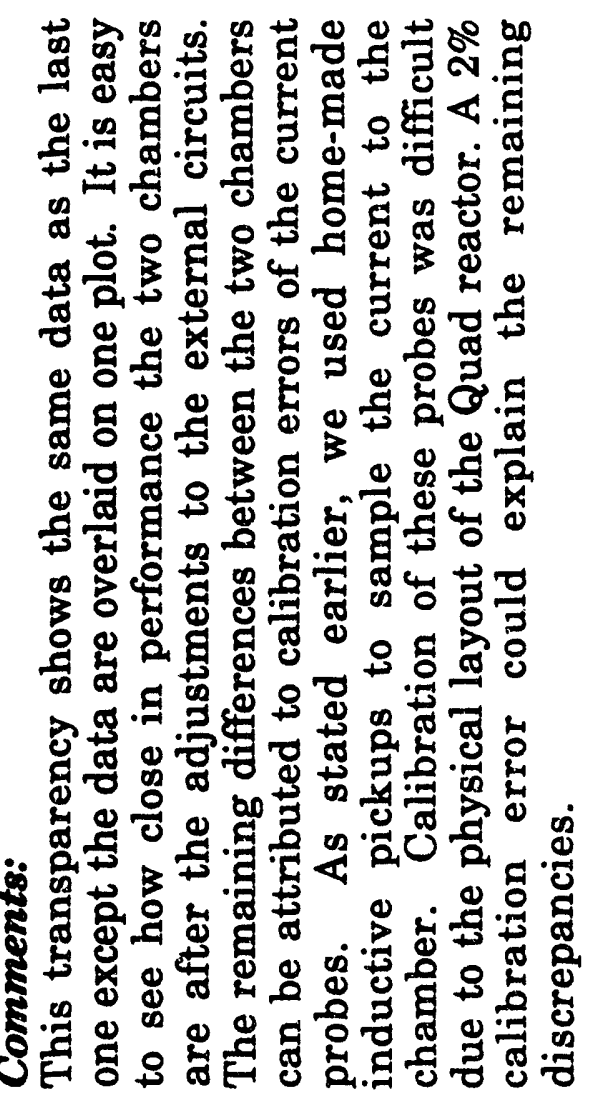




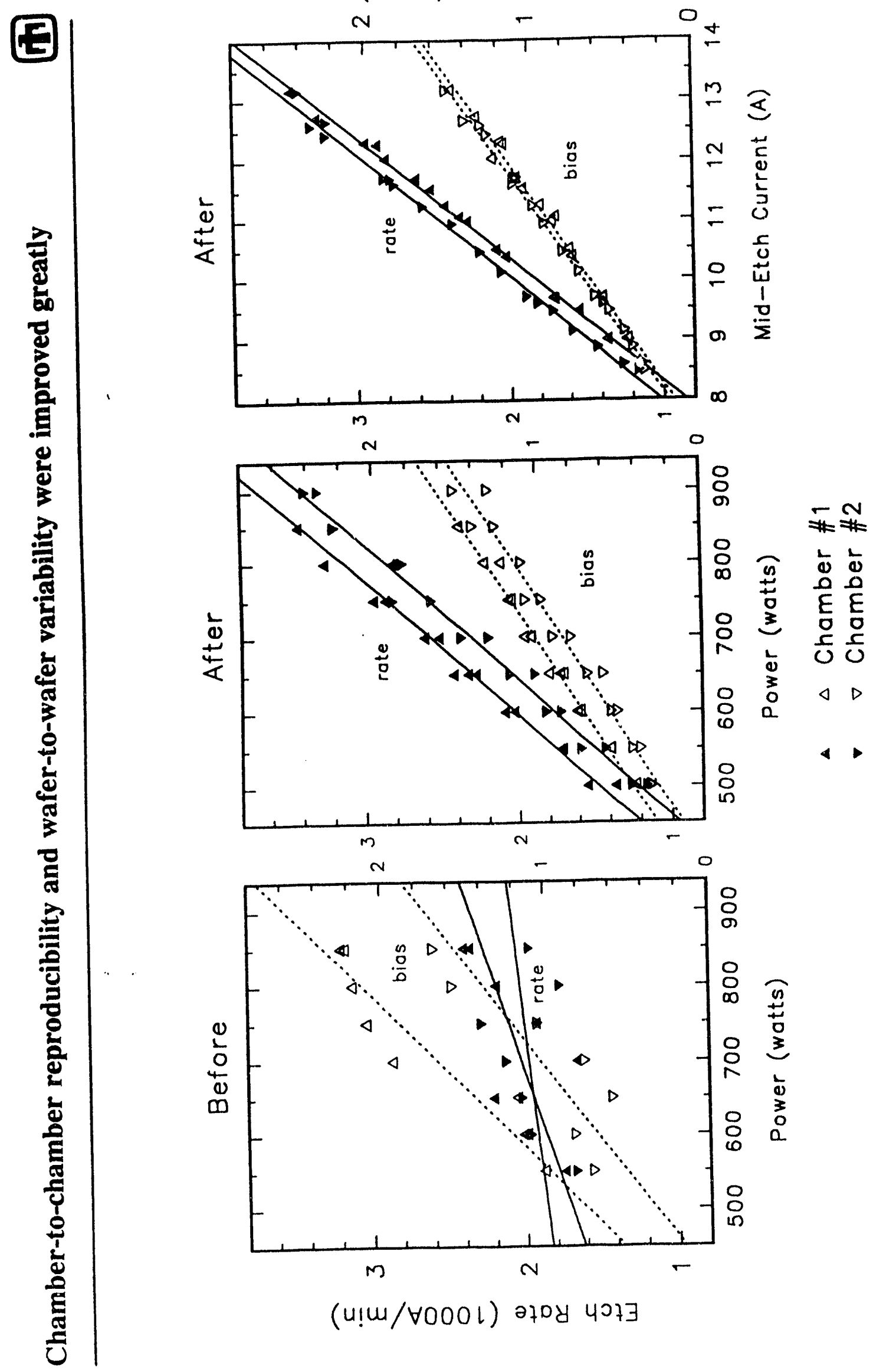




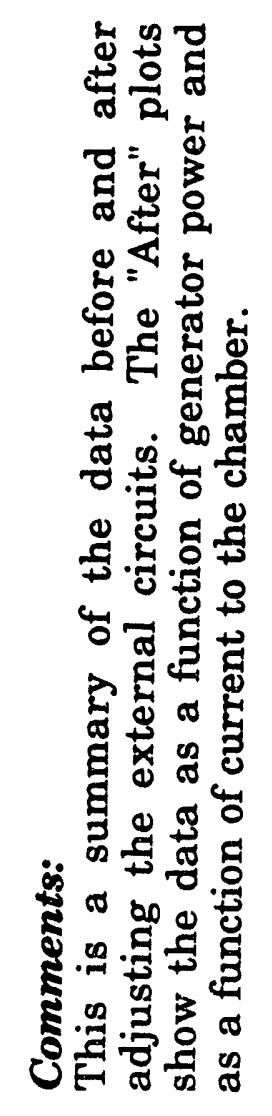




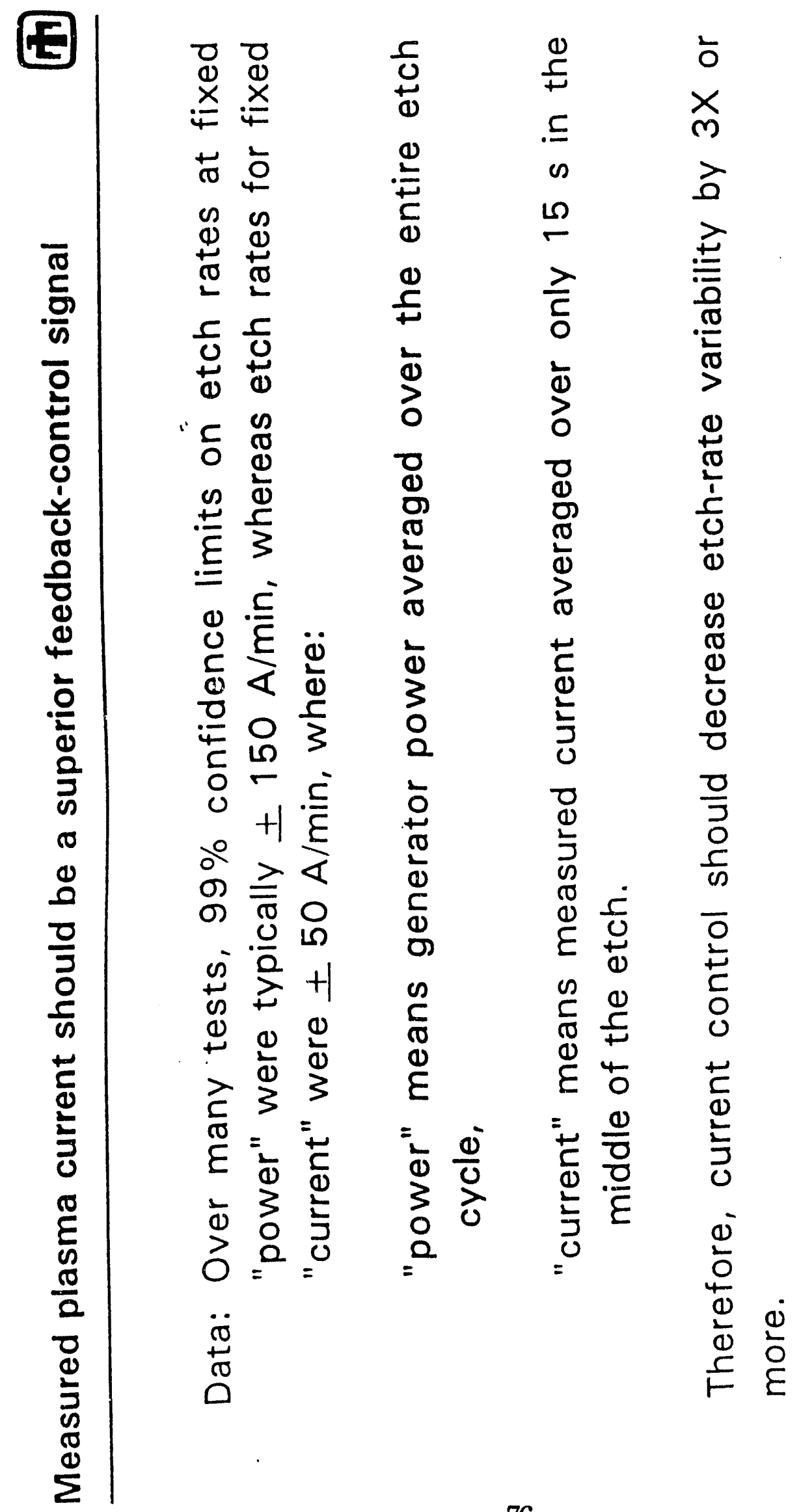




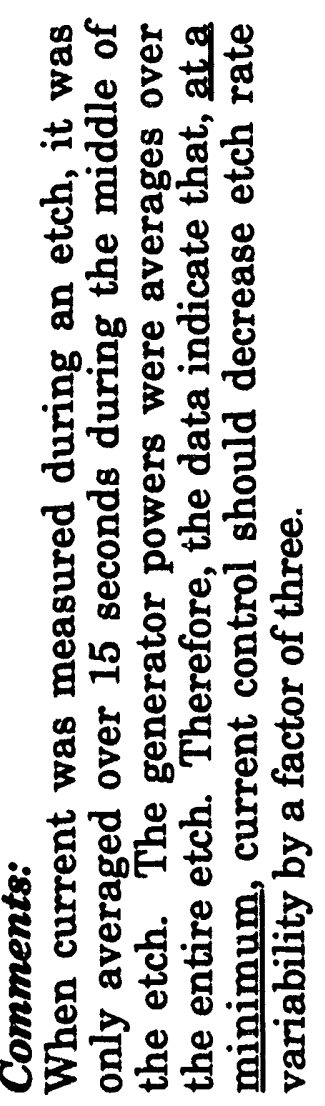



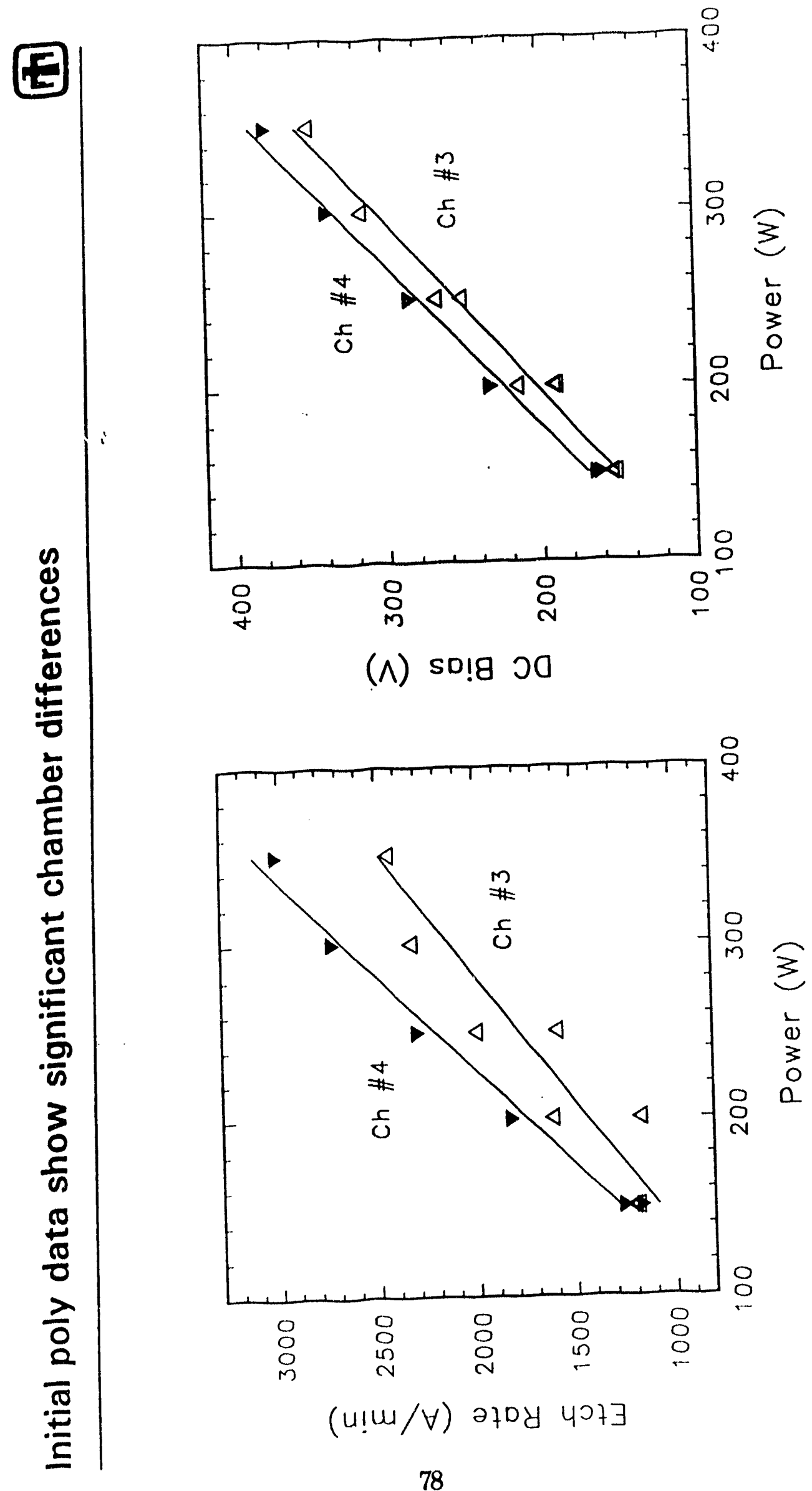


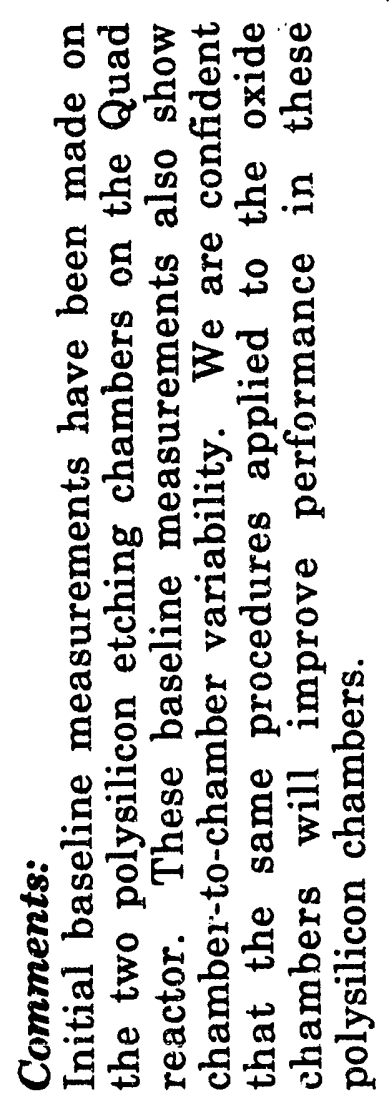




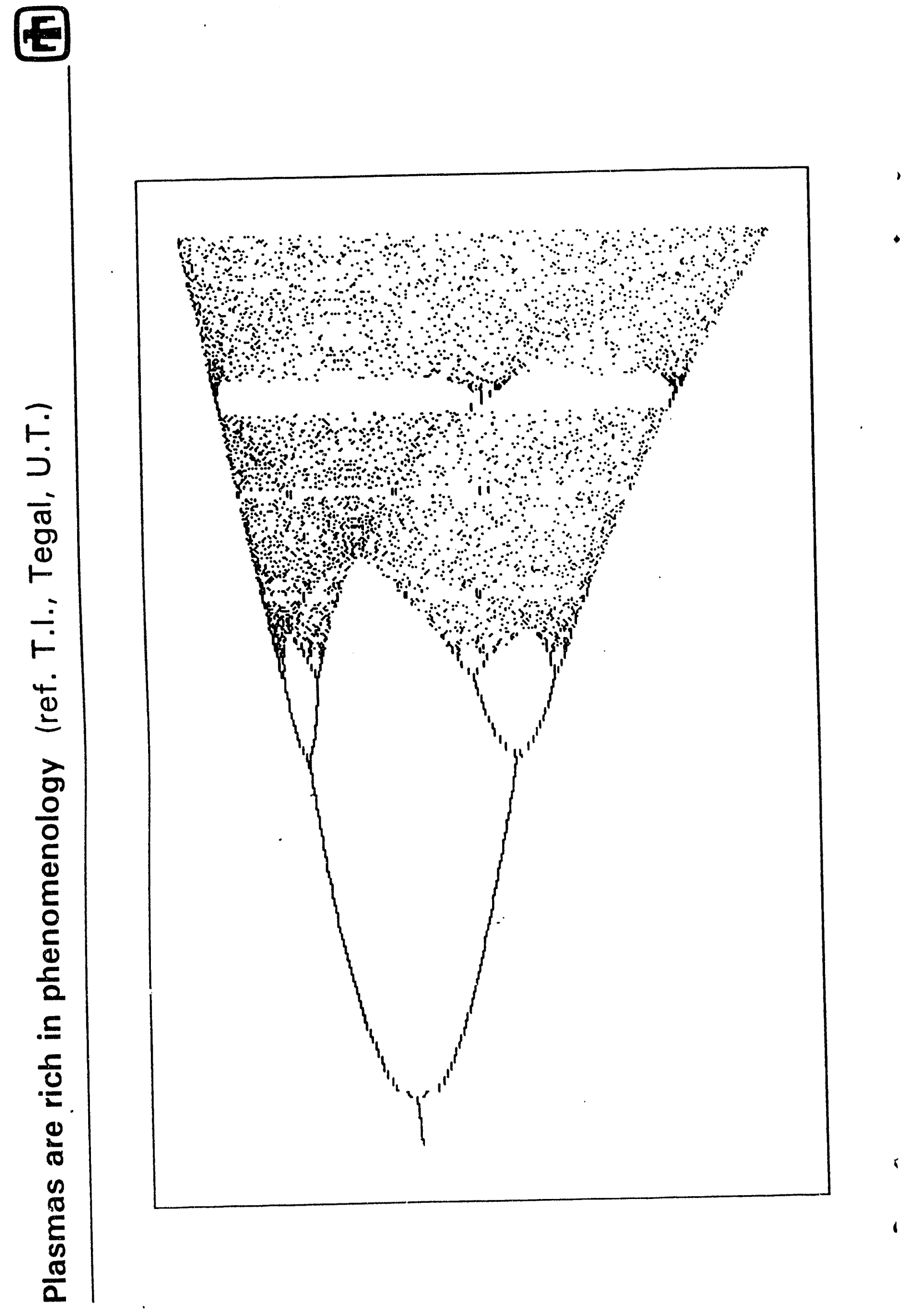




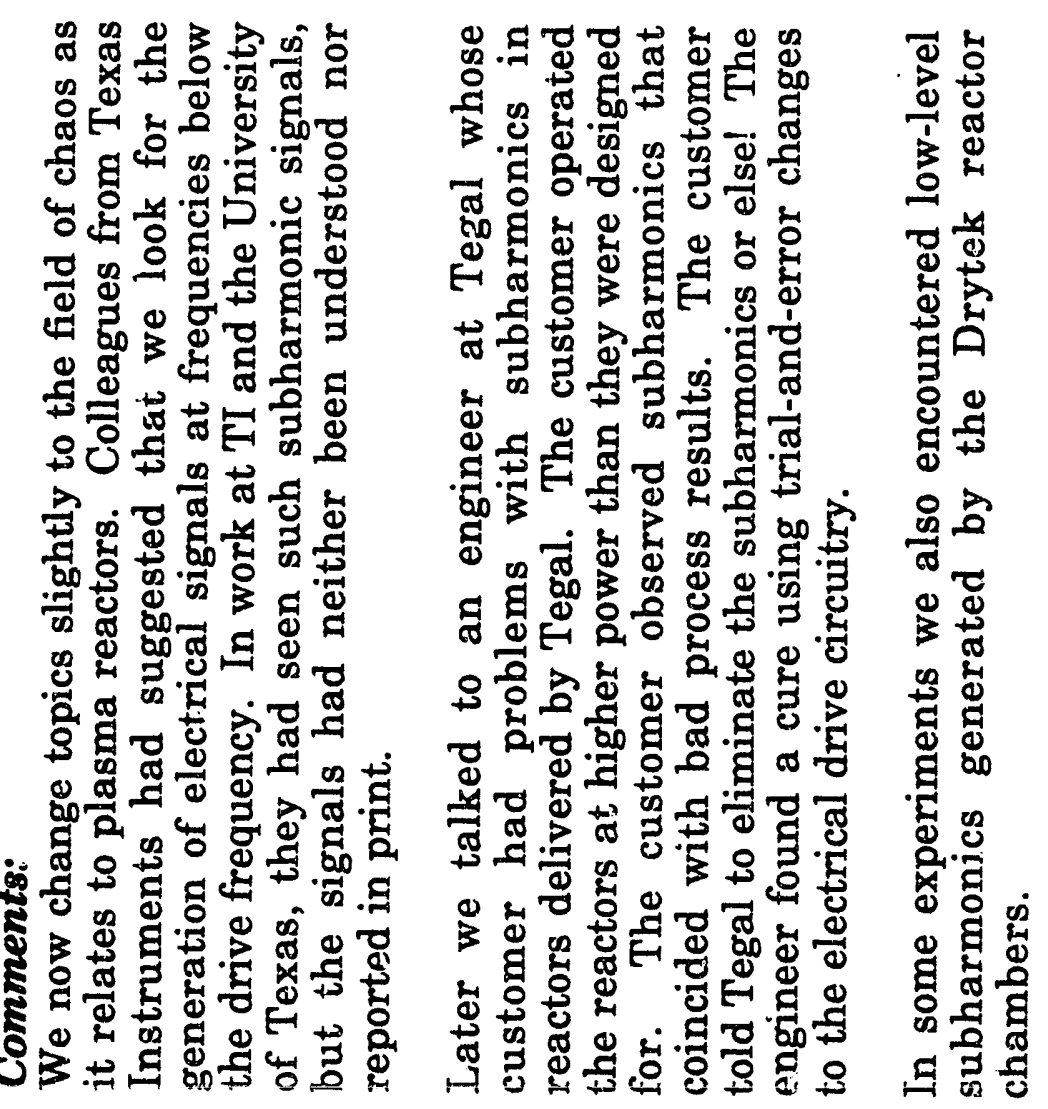




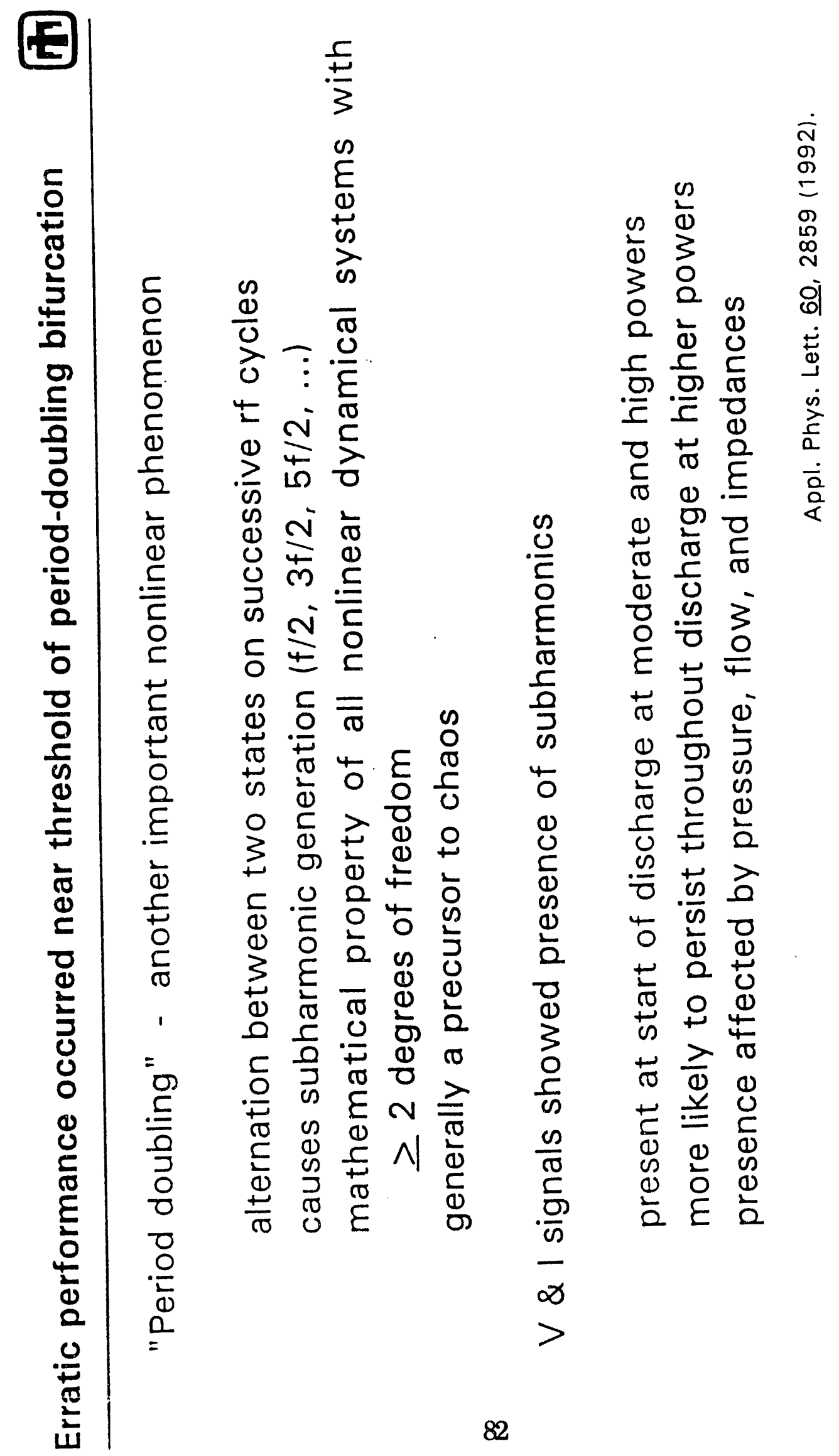




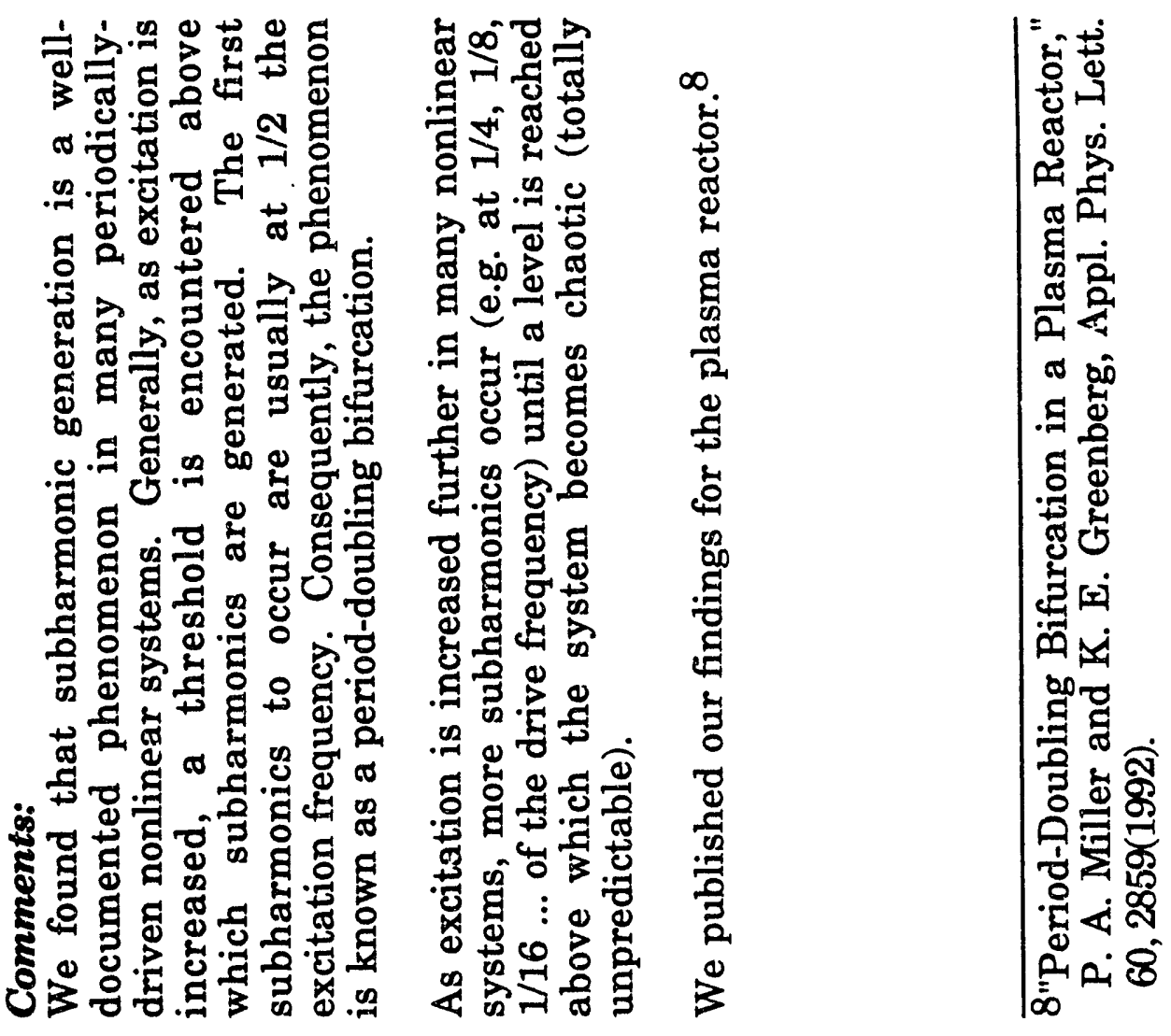



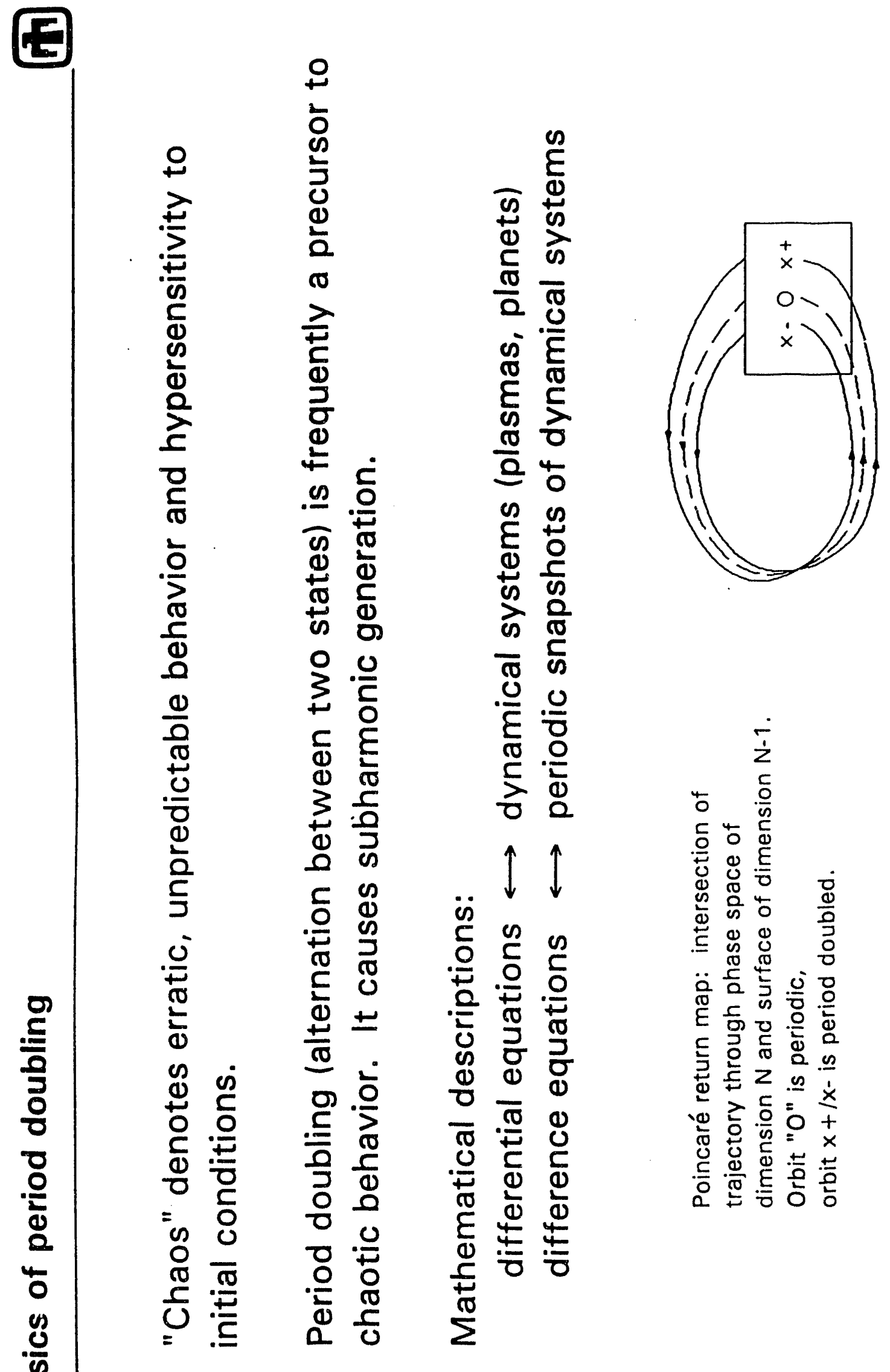


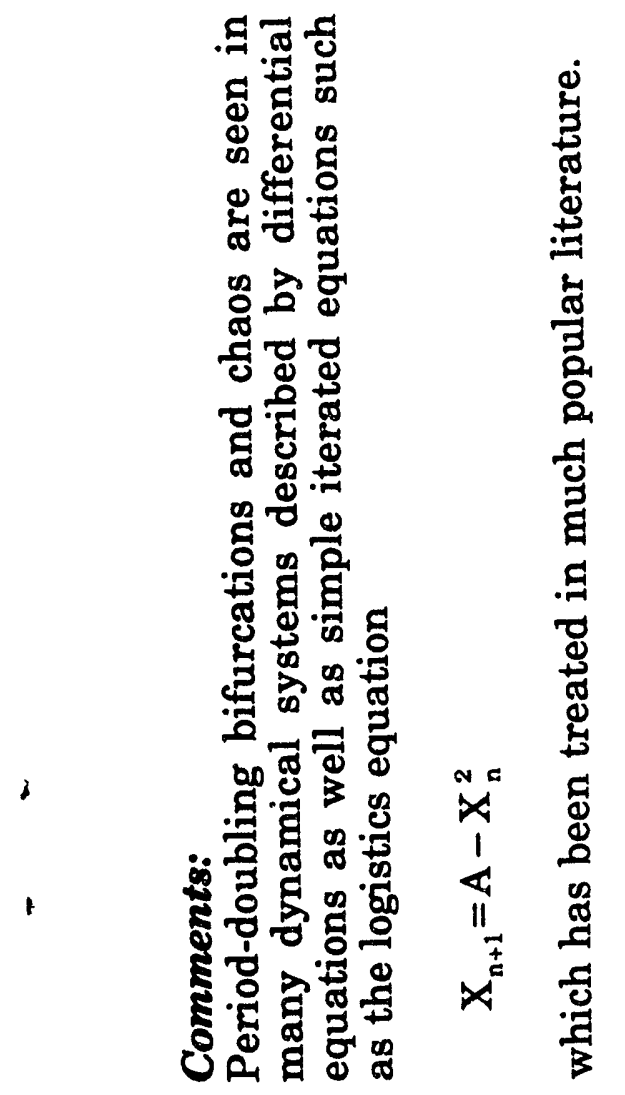




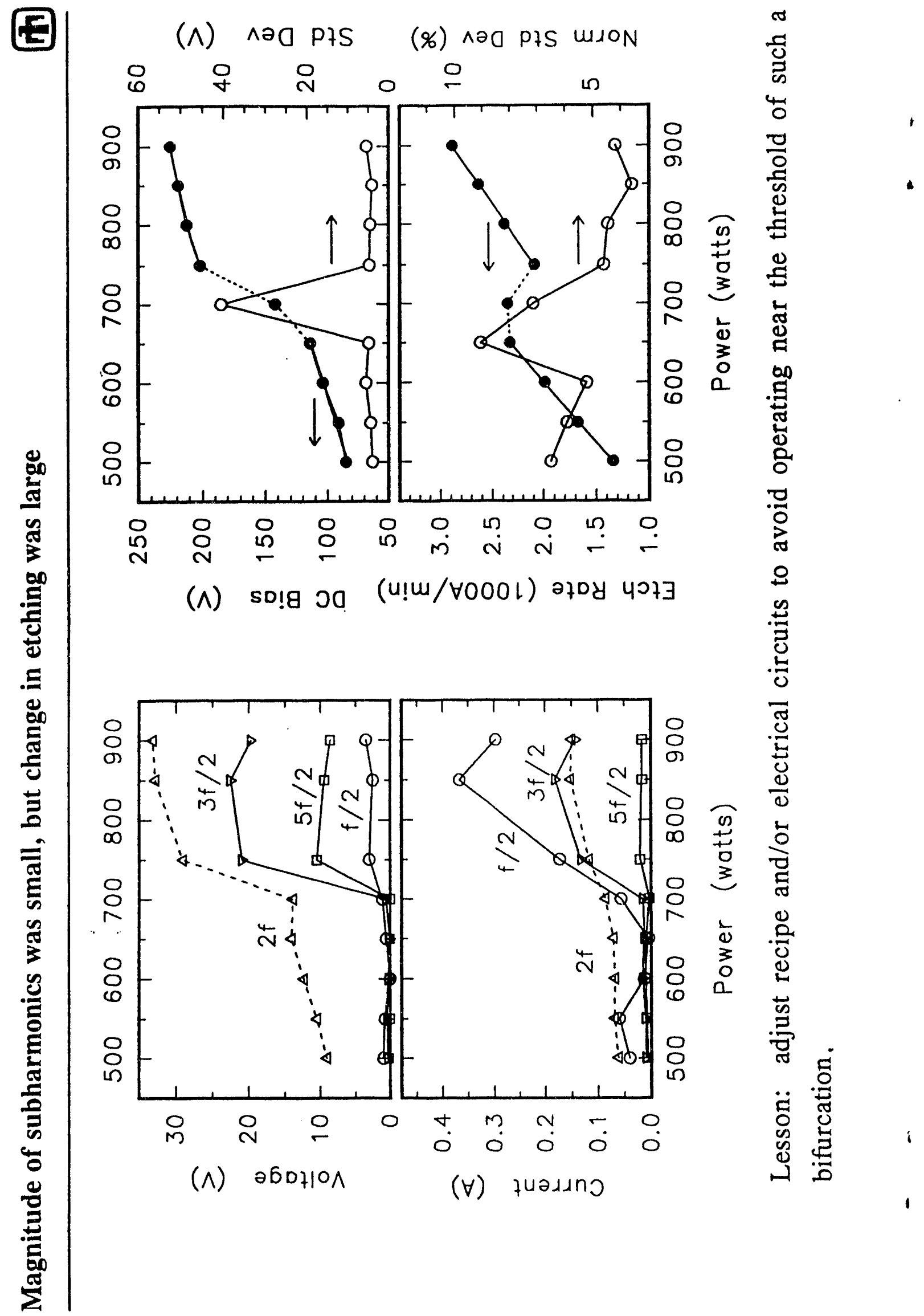



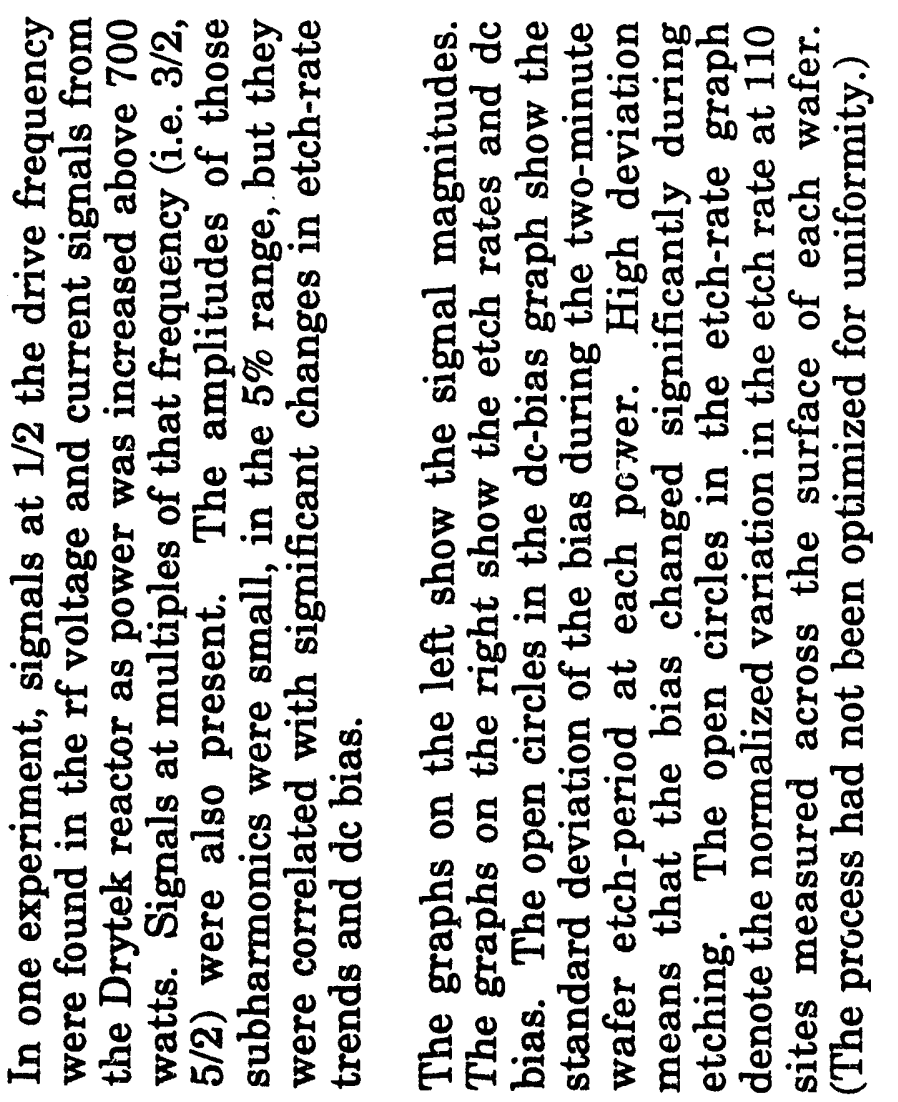
. क 0 .

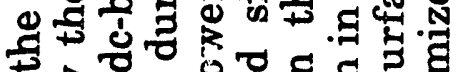
†

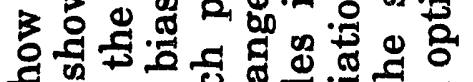

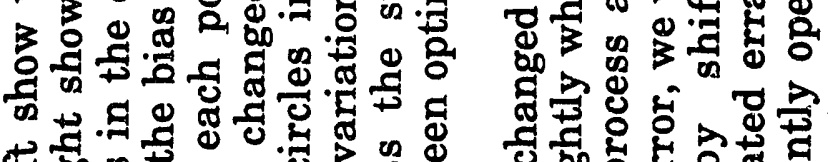
屯

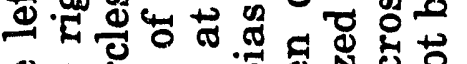

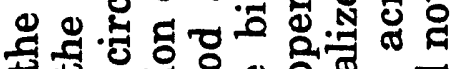

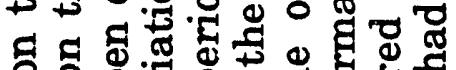

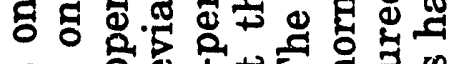

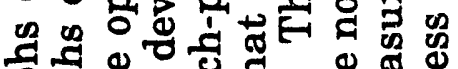

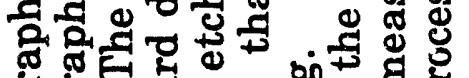
क्ष क क

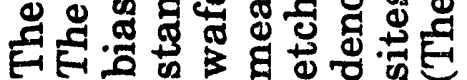

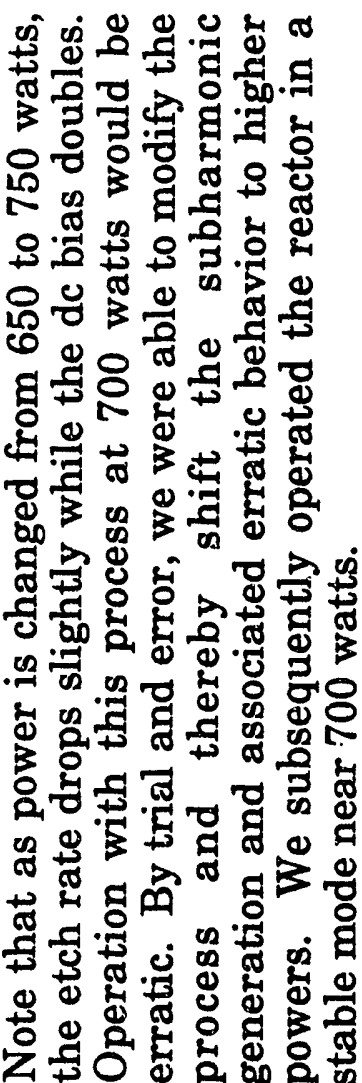




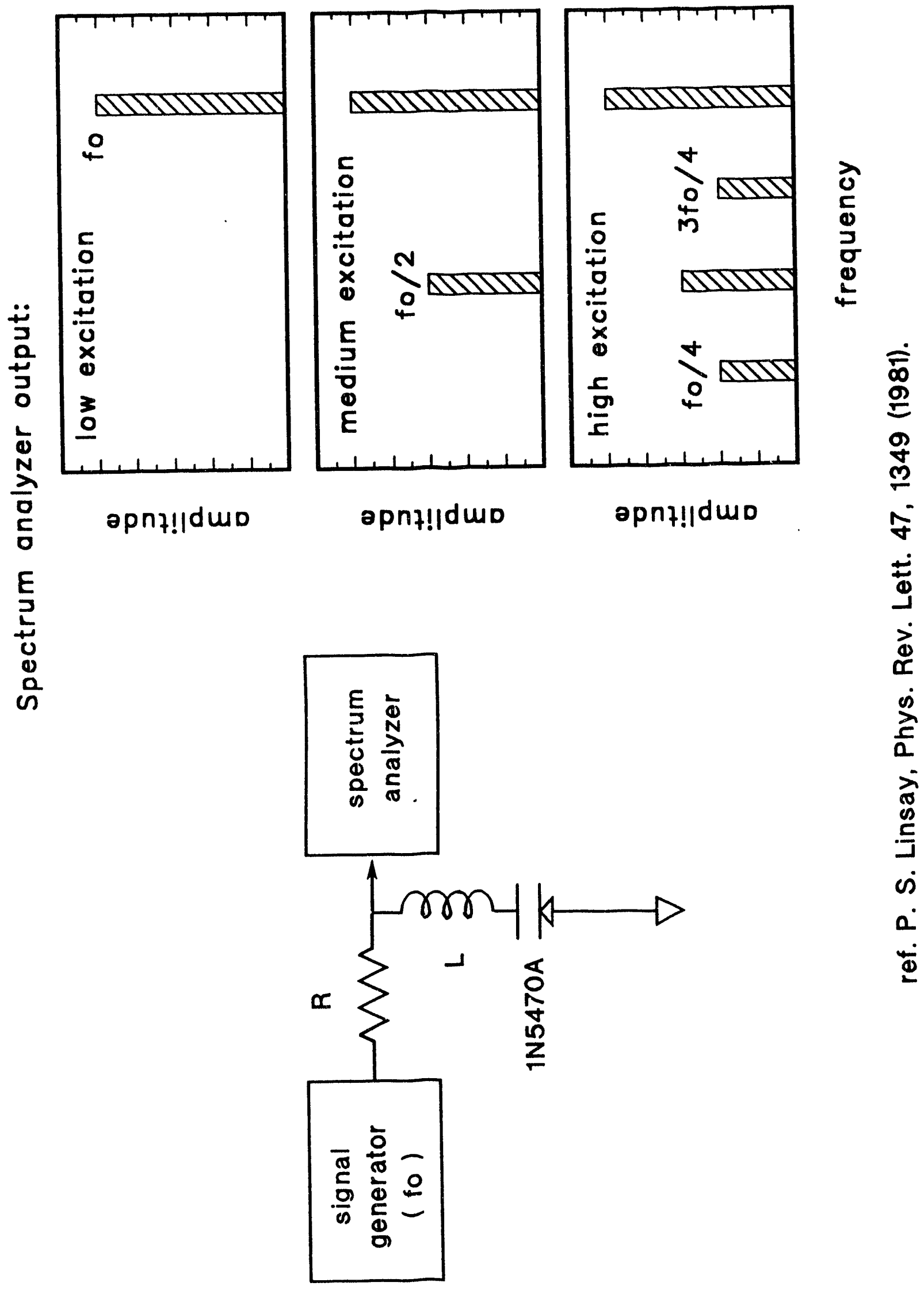




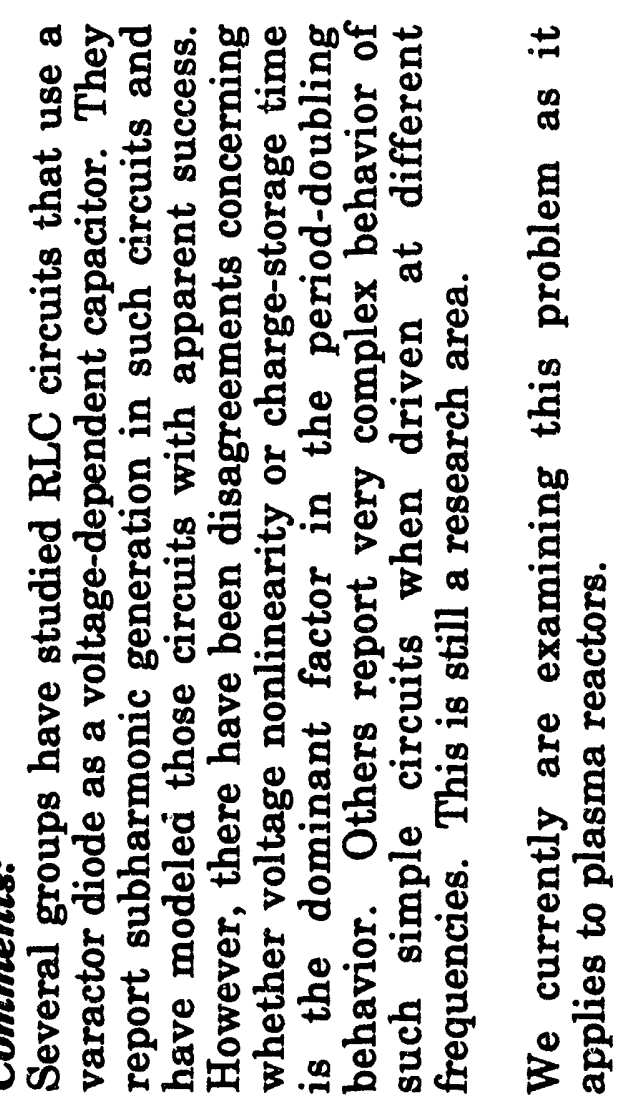


ธ

ธิ

$\frac{7}{\frac{1}{0}}$

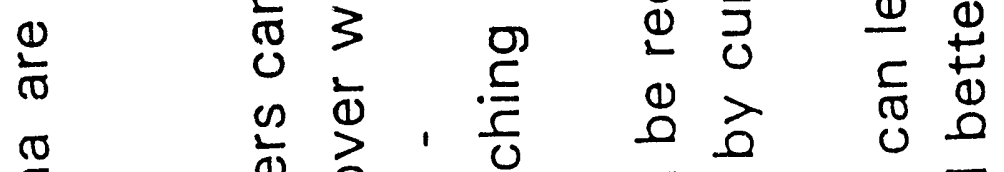

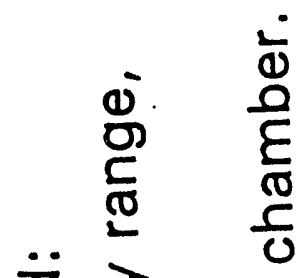

बें है

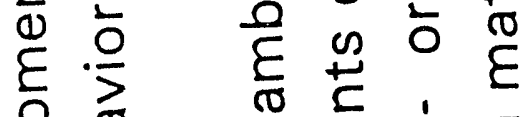

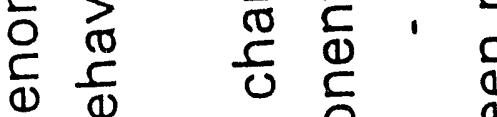

舀

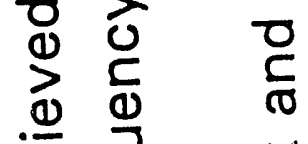

定

ע

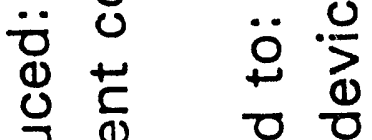

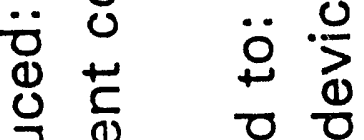

$\sum_{\substack{0 \\ \hdashline}}^{0}$

$\frac{\pi}{\frac{0}{0}}$

ป

ร

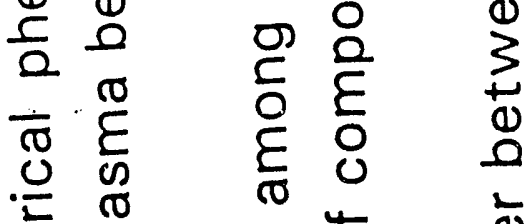

$\geqq \frac{1}{0}$

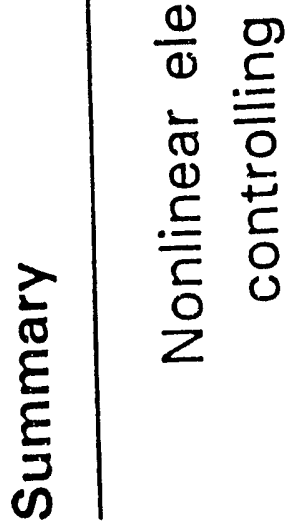

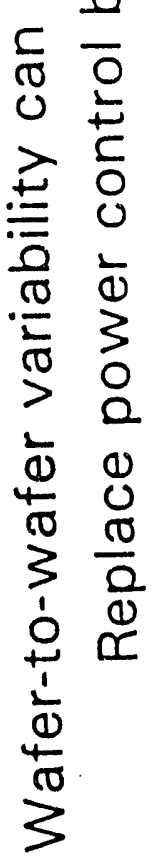

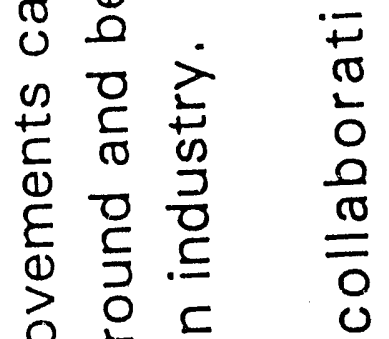

인 응.

ํㅡㄴ 을

¿

क

m

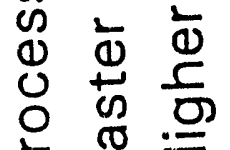

뉸 觉

$\stackrel{Ð}{\infty}$ 


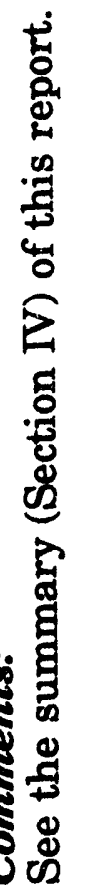




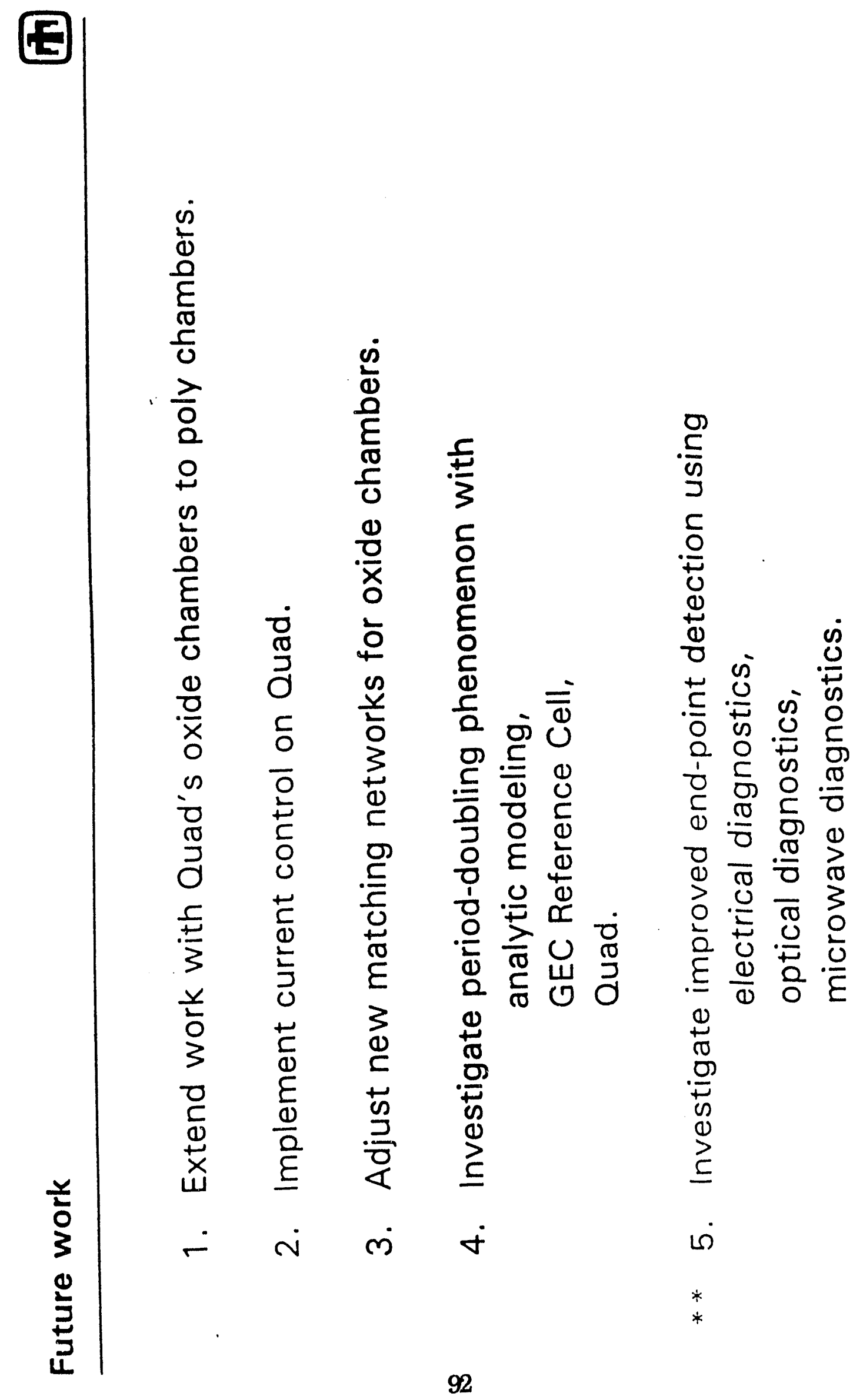

